NASA Contractor Report 191119

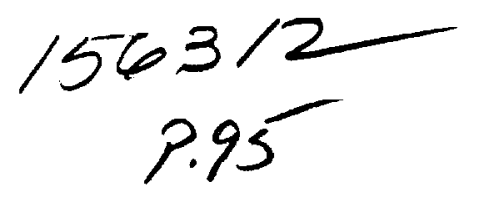

\title{
Ionospheric Plasma Flow About a System of Electrically Biased Flat Plates
}

Joel L. Herr

Sverdrup Technology, Inc.

Lewis Research Center Group

Brook Park, Ohio

April 1993

Prepared for

Lewis Research Center

Under Contract NAS3-25266

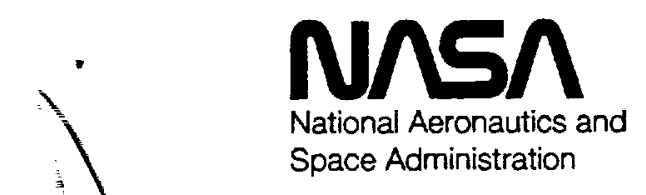

(NASA-CR-191119) IONDSPHERIC

N93-23131

PLASMA FLOW ABOUT A SYSTEM OF

ELECTRICALLY BIASED FLAT PLATES

M.S. Thesis - Cleveland State

Unclas

univ. Final Report (Sverdrup

Technology) $95 \mathrm{p}$

$63 / 18 \quad 0156312$ 


\section{IONOSPHERIC PLASMA FLOW ABOUT A SYSTEM OF \\ ELECTRICALLY BIASED FLAT PLATES \\ Joel L. Herr \\ Sverdrup Technology, Inc. \\ Lewis Research Center Group \\ Brook Park, Ohio 44142}

\section{ABSTRACT}

The steady state interaction of two electrically biased parallel plates immersed in a flowing plasma characteristic of low earth orbit is studied numerically. Fluid equations are developed to describe the motion of the cold positively charged plasma ions, and are solved using finite-differences in two dimensions on a Cartesian grid. The behavior of the plasma electrons is assumed to be described by the MaxwellBoltzmann distribution.

Results are compared to an analytical and a particle simulation technique for a simplified flow geometry consisting of a single semi-infinite negatively biased plate. Comparison of the extent of the electrical disturbance into the flowing plasma and the magnitude of the current collected by the plate is very good.

The interaction of two equally biased parallel plates is studied as a function of applied potential. The separation distance at which the current collected by either plate 
decreases by five and twenty percent is determined as a function of applied potential. The percent decreases were based on a non-interacting case. The decrease in overall current is caused by a decrease in ionic density in the region between the plates. As the separation between the plates decreases, the plates collect the ions at a faster rate than they are supplied to the middle region by the oncoming plasma flow.

The docking of spacecraft in orbit is simulated by moving two plates of unequal potential toward one another in a quasistatic manner. one plate is held at a large negative potential while the other floats electrically in the resulting potential field. It is found that the floating plate does not charge continuously negative as it approaches the other more negatively biased plate. Instead, it charges more and then less negative as ionic current decreases and then increases respectively upon approach. When the two plates come into contact, it is expected that the electrically floating plate will charge rapidly negative to a potential near that of the other plate. 


\section{NOMENCLATURE}

Symbol

Description

Units

k

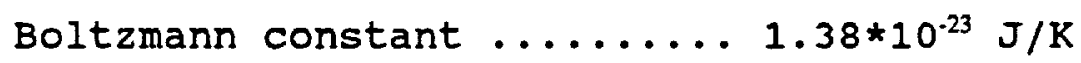

$R_{0} \ldots \ldots \ldots$ characteristic length ..... m

q $\ldots \ldots \ldots$ charge $\ldots \ldots \ldots \ldots \ldots \ldots \ldots$

$\nu \ldots \ldots \ldots$ collision frequency $\ldots \ldots \ldots \mathrm{s}^{-1}$

j ........ current density ......... A $/ \mathrm{m}^{2}$

$\lambda_{\mathrm{D}} \ldots \ldots \ldots$ Debye length $\ldots \ldots \ldots \ldots \ldots$

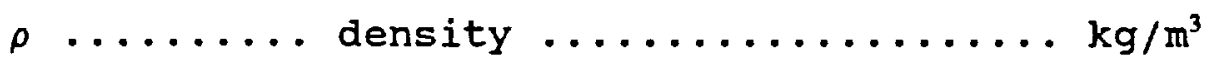

E ....... electric field vector $\ldots . . . \mathrm{V} / \mathrm{m}$

$\phi \ldots \ldots . .$. electric potential ....... V

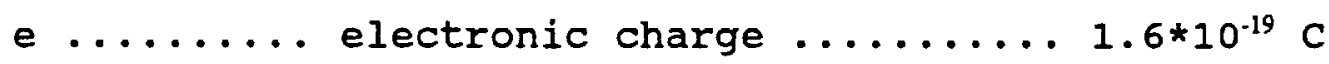

$\mathbf{r} \ldots \ldots \ldots$ flux density $\ldots \ldots \ldots \ldots \mathrm{m}^{-2} \mathrm{~s}^{-1}$

$\mathrm{u}_{\beta} \ldots \ldots \ldots$ ion sound speed $\ldots \ldots \ldots \ldots . . . \mathrm{m} / \mathrm{s}$

m $\ldots \ldots \ldots$ mass $\ldots \ldots \ldots \ldots \ldots \ldots \ldots \ldots \ldots$

$\mu \ldots \ldots \ldots$ mobility $\ldots \ldots \ldots \ldots \ldots \ldots \ldots$ cs $/ \mathrm{kg}$

n $\ldots \ldots \ldots$ number density $\ldots \ldots \ldots \ldots \mathrm{m}^{-3}$

$\mathrm{L}_{\text {TOT }} \ldots \ldots$ plate length $\ldots \ldots \ldots \ldots$

p $\ldots \ldots \ldots$ pressure $\ldots \ldots \ldots \ldots \ldots \ldots$ N $/ \mathrm{m}^{2}$

s $\ldots \ldots \ldots$ sheath dimension ......... m

T....... temperature $\ldots \ldots \ldots \ldots \ldots$......

v...... velocity vector $\ldots . \ldots \ldots . . .6 \mathrm{~m} / \mathrm{s}$

u ........ $x$-component of velocity .... m/s

v ........ y-component of velocity .... m/s

D ........ free diffusion coefficient .. $\mathrm{m}^{2} / \mathrm{s}$

$\varepsilon_{0} \ldots \ldots$ permittivity of free space $\ldots 8.854 * 10^{-12} \mathrm{C}^{2} / \mathrm{Jm}$ 


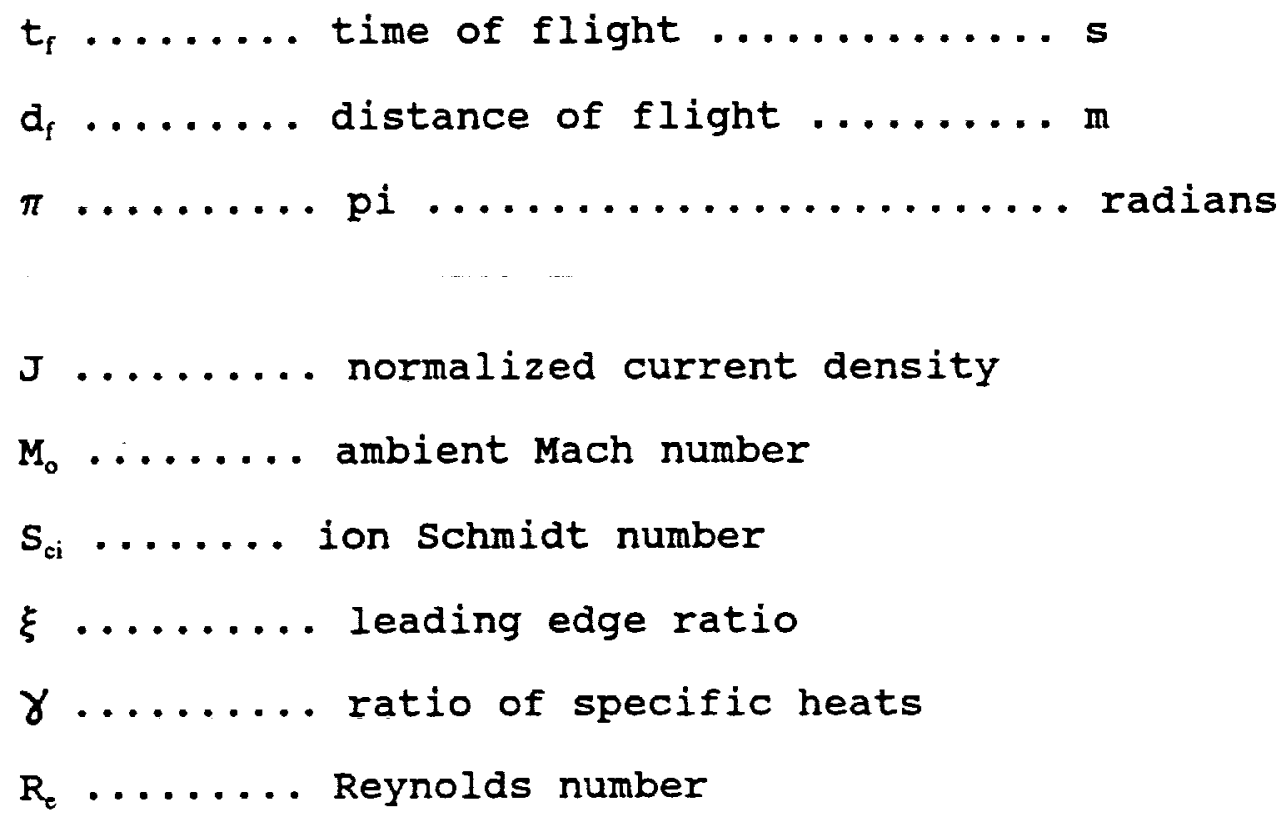

Subscripts

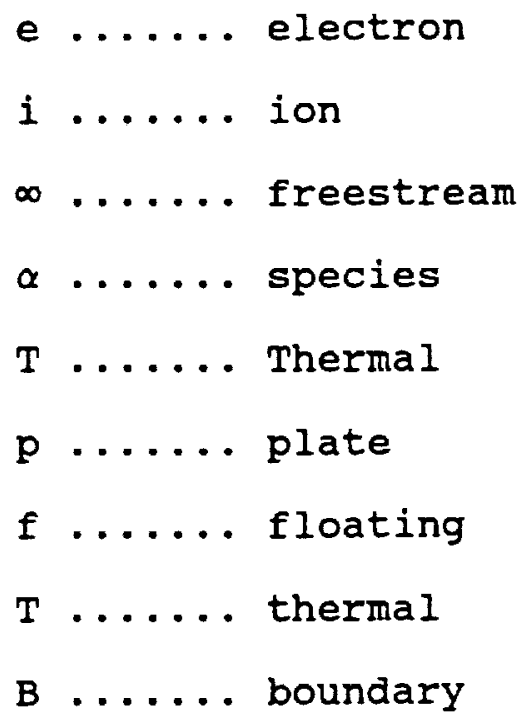

Superscripts

s ...... sheath 
ACKNOWLEDGMENTS..............................

ABSTRACT .............................

NOMENCLATURE..............................

TABLE OF CONTENTS..................................

LIST OF FIGURES.........................

LIST OF TABLES...........................

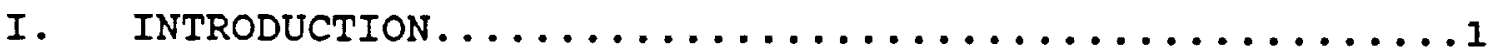

II. BACKGROUND...........................

Continuum Probe Theory.................8

Ionospheric Aerodynamics.................. 13

III. APPROACH AND FORMULATION. ................... 19

Assumptions.............................. 19

Governing Equations..................21

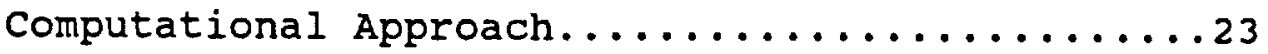

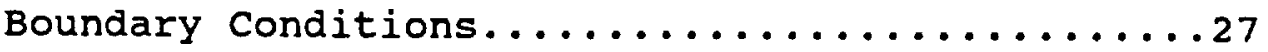

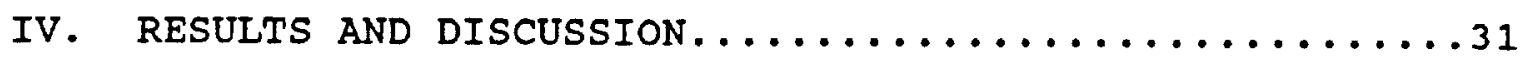

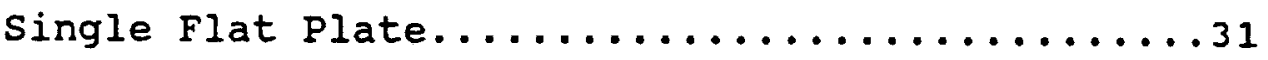

Analytical Comparison...................

Floating Potential.................40

Computational Comparison.............43

Two Parallel Plates....................43

Minimum Separation Distance..........43

Spacecraft Docking.......................

v. CONCLUSION............................

REFERENCES.............................. . . . 


\section{LIST OF FIGURES}

Figure

Page

1. Schematic of a two-plate geometry

2. Variable grid structure for a two-plate geometry

3. $u$ velocity contours for a single semiinfinite flat plate: $u_{\infty}=8780 \mathrm{~m} / \mathrm{s}, T_{e}=0.2 \mathrm{eV}$, $\phi_{\mathrm{p}}=-20, \mathrm{~m}_{\mathrm{i}}=16$ amu; Contour values:

$\mathrm{u} / \mathrm{u}_{\infty}=1.0001,1.01,1.05,1.1,1.15$ from the freestream toward the plate

4. $v$ velocity contours for a single semi-

infinite flat plate: $u_{\infty}=8780 \mathrm{~m} / \mathrm{s}, \mathrm{T}_{\mathrm{e}}=0.2 \mathrm{eV}$, $\phi_{\mathrm{p}}=-20, m_{\mathrm{i}}=16$ amu; Contour values:

$\mathrm{v} / \mathrm{u}_{\infty}= \pm .01, \pm .1, \pm .3, \pm .5$, positive values

below, negative values above, from the

freestream toward the plate

5. Ionic density contours for a single semi-

infinite flat plate: $u_{\infty}=8780 \mathrm{~m} / \mathrm{s}, \mathrm{T}_{\mathrm{e}}=0.2 \mathrm{eV}$, $\phi_{\mathrm{p}}=-20, \mathrm{~m}_{\mathrm{i}}=16$ amu; Contour values:

$\mathrm{n}_{\mathrm{i}} / \mathrm{n}_{\infty}=.99, .7, .368, .2, .1$, from the

freestream toward the plate

6. Electric potential contours for a single

semi-infinite flat plate: $u_{\infty}=8780 \mathrm{~m} / \mathrm{s}$, $\mathrm{T}_{\mathrm{c}}=0.2 \mathrm{eV}, \phi_{\mathrm{p}}=-20, \mathrm{~m}_{\mathrm{i}}=16$ amu; Contour

values: $\phi=-.1,-1,-5,-10,-15$, from

the freestream toward the plate

7. Streamlines superimposed on electric potential contours for a single semiinfinite flat plate: $u_{\infty}=8780 \mathrm{~m} / \mathrm{s}$,

$\mathrm{T}_{\mathrm{e}}=0.2 \mathrm{eV}, \phi_{\mathrm{p}}=-20, \mathrm{~m}_{\mathrm{i}}=16 \mathrm{amu}$

8. Normalized current density $\mathrm{J} /\left(\mathrm{en}_{\infty} \mathrm{u}_{\beta}\right)$ to

a single semi-infinite flat plate:

$\mathrm{u}_{\infty}=8780 \mathrm{~m} / \mathrm{s}, \mathrm{T}_{\mathrm{c}}=0.2 \mathrm{eV}, \phi_{\mathrm{p}}=-20, \mathrm{~m}_{\mathrm{i}}=16 \mathrm{amu}$

9. Minimum interaction separation distance between two equally biased flat plates as a function of applied potential: $u_{\infty}=7800 \mathrm{~m} / \mathrm{s}$, $\mathrm{T}_{\mathrm{c}}=0.2 \mathrm{eV}, \mathrm{d}_{\mathrm{p}}=5 \lambda_{\mathrm{D}}, \mathrm{m}_{\mathrm{i}}=16$ amu 


\section{LIST OF FIGURES (CONTINUED)}

Figure

Page

10. u velocity contours for two equally

biased flat plates: $d_{s e p}=29 \lambda_{D}, d_{P}=5 \lambda_{D}$, $\phi_{\mathrm{p}}=-50, \mathrm{u}_{\infty}=7800 \mathrm{~m} / \mathrm{s}, \mathrm{T}_{\mathrm{e}}=0.2 \mathrm{eV}, \mathrm{m}_{\mathrm{i}}=16 \mathrm{amu}$ Contour values: $u / u_{\infty}=1.005,1.05,1.1,1.3$, 1.5 from the freestream toward the plates

11. $v$ velocity contours for two equally biased

flat plates: $d_{s c p}=29 \lambda_{D}, d_{p}=5 \lambda_{D}, \phi_{p}=-50$, $u_{\infty}=7800 \mathrm{~m} / \mathrm{s}, T_{c}=0.2 \mathrm{eV}, m_{i}=16$ amu; Contour values: $v / u_{\infty}= \pm .01, \pm .1, \pm .3, \pm .5, \pm 1$ positive values below the plates, negative values above from the freestream toward the plates

12. Ionic density contours for two equally biased flat plates: $d_{\text {sep }}=29 \lambda_{D}, d_{p}=5 \lambda_{D}, \phi_{p}=-50$, $\mathrm{u}_{\infty}=7800 \mathrm{~m} / \mathrm{s}, \mathrm{T}_{\mathrm{e}}=0.2 \mathrm{eV}, \mathrm{m}_{\mathrm{i}}=16 \mathrm{amu}$; Contour values: $n_{\mathrm{i}} / \mathrm{n}_{\infty}=.95, .75, .5$, from the freestream toward the plates

13. Electric potential contours for two equally biased flat plates: $d_{s e p}=29 \lambda_{D}, d_{p}=5 \lambda_{D}, \phi_{p}=-50$, $u_{\infty}=7800 \mathrm{~m} / \mathrm{s}, \mathrm{T}_{\mathrm{e}}=0.2 \mathrm{eV}, \mathrm{m}_{\mathrm{i}}=16$ amu; Contour values: $\phi=-.1,-1,-10,-20,-30$ from the freestream toward the plates

14. $u$ velocity contours for two equally biased flat plates: $d_{s e p}=19 \lambda_{D}, d_{p}=5 \lambda_{D}, \phi_{p}=-50$, $u_{\infty}=7800 \mathrm{~m} / \mathrm{s}, T_{\mathrm{e}}=0.2 \mathrm{eV}, \mathrm{m}_{\mathrm{i}}=16$ amu; Contour values: $u / u_{\infty}=1.005,1.05,1.1,1.3,1.5$ from the freestream toward the plates

15. $v$ velocity contours for two equally biased flat plates: $d_{s e p}=19 \lambda_{D}, d_{p}=5 \lambda_{D}, \phi_{p}=-50$, $\mathrm{u}_{\infty}=7800 \mathrm{~m} / \mathrm{s}, \mathrm{T}_{\mathrm{e}}=0.2 \mathrm{eV}, \mathrm{m}_{\mathrm{i}}=16$ amu; Contour values: $v / u_{\infty}= \pm .01, \pm .1, \pm .3, \pm .5, \pm 1$ positive values below the plates, negative values above from the freestream toward the plates

16. Ionic density contours for two equally biased

flat plates: $d_{\text {sep }}=19 \lambda_{D}, d_{p}=5 \lambda_{D}, \phi_{P}=-50$, $\mathrm{u}_{\infty}=7800 \mathrm{~m} / \mathrm{s}, \mathrm{T}_{\mathrm{e}}=0.2 \mathrm{eV}, \mathrm{m}_{\mathrm{i}}=16$ amu; Contour values: $n_{\mathrm{i}} / \mathrm{n}_{\infty}=.95, .75, .5$, from the freestream toward the plates 


\section{LIST OF FIGURES (CONTINUED)}

Figure

Page

17. Electric potential contours for two equally

biased flat plates: $d_{s e p}=19 \lambda_{D}, d_{p}=5 \lambda_{D}, \phi_{p}=-50$, $\mathrm{u}_{\infty}=7800 \mathrm{~m} / \mathrm{s}, \mathrm{T}_{\mathrm{c}}=0.2 \mathrm{eV}, \mathrm{m}_{\mathrm{i}}=16 \mathrm{amu}$; Contour values: $\phi=-.1,-1,-10,-20,-30$ from the freestream toward the plates

18. u velocity contours for two equally biased

flat plates: $d_{\text {sep }}=14 \lambda_{D}, d_{p}=5 \lambda_{D}, \phi_{p}=-50$, $\mathrm{u}_{\infty}=7800 \mathrm{~m} / \mathrm{s}, \mathrm{T}_{\mathrm{c}}=0.2 \mathrm{eV}, \mathrm{m}_{\mathrm{i}}=16 \mathrm{amu}$; Contour values: $u / u_{\infty}=1.005,1.05,1.1,1.3,1.5$ from the freestream toward the plates

19. $v$ velocity contours for two equally biased flat plates: $d_{\text {sep }}=14 \lambda_{D}, d_{p}=5 \lambda_{D}, \phi_{p}=-50$, $u_{\infty}=7800 \mathrm{~m} / \mathrm{s}, \mathrm{T}_{\mathrm{e}}=0.2 \mathrm{eV}, \mathrm{m}_{\mathrm{i}}=16 \mathrm{amu}$; Contour values: $\mathrm{v} / \mathrm{u}_{\infty}= \pm .01, \pm .1, \pm .3, \pm .5, \pm 1$ positive values below the plates, negative values above from the freestream toward the plates

20. Ionic density contours for two equally biased

flat plates: $d_{s e p}=14 \lambda_{D}, d_{p}=5 \lambda_{D}, \phi_{p}=-50$, $\mathrm{u}_{\infty}=7800 \mathrm{~m} / \mathrm{s}, \mathrm{T}_{\mathrm{e}}=0.2 \mathrm{eV}, \mathrm{m}_{\mathrm{i}}=16$ amu; Contour values: $n_{i} / n_{\infty}=.95, .75, .5, .45$ from the freestream toward the plates

21. Electric potential contours for two equally biased flat plates: $d_{s c p}=14 \lambda_{D}, d_{p}=5 \lambda_{D}, \phi_{p}=-50$, $u_{\infty}=7800 \mathrm{~m} / \mathrm{s}, \mathrm{T}_{\mathrm{e}}=0.2 \mathrm{eV}, \mathrm{m}_{\mathrm{i}}=16$ amu; Contour values: $\phi=-.1,-1,-10,-20,-30$ from the freestream toward the plates

22. $u$ velocity contours for two equally biased flat plates: $d_{\text {sep }}=9 \lambda_{D}, d_{p}=5 \lambda_{D}, \phi_{p}=-50$, $\mathrm{u}_{\infty}=7800 \mathrm{~m} / \mathrm{s}, \mathrm{T}_{\mathrm{e}}=0.2 \mathrm{eV}, \mathrm{m}_{\mathrm{i}}=16 \mathrm{amu}$; Contour values: $u / u_{\infty}=1.005,1.05,1.1,1.3,1.5$ from the freestream toward the plates

23. $v$ velocity contours for two equally biased flat plates: $d_{\text {sep }}=9 \lambda_{D}, d_{p}=5 \lambda_{D}, \phi_{p}=-50$, $\mathrm{u}_{\infty}=7800 \mathrm{~m} / \mathrm{s}, \mathrm{T}_{\mathrm{e}}=0.2 \mathrm{eV}, \mathrm{m}_{\mathrm{i}}=16 \mathrm{amu}$; Contour values: $v / u_{\infty}= \pm .01, \pm .1, \pm .3, \pm .5, \pm 1$ positive values below the plates, negative values above from the freestream toward the plates 
24. Ionic density contours for two equally biased flat plates: $d_{\text {sep }}=9 \lambda_{\mathrm{D}}, \mathrm{d}_{\mathrm{p}}=5 \lambda_{\mathrm{D}}, \phi_{\mathrm{p}}=-50$, $u_{\infty}=7800 \mathrm{~m} / \mathrm{s}, \mathrm{T}_{\mathrm{c}}=0.2 \mathrm{eV}, \mathrm{m}_{\mathrm{i}}=16 \mathrm{amu}$; Contour values: $n_{\mathrm{i}} / \mathrm{n}_{\infty}=.95, .75, .5, .45$ from the freestream toward the plates

25. Electric potential contours for two equally biased flat plates: $d_{\iota c p}=9 \lambda_{D}, d_{p}=5 \lambda_{D}, \phi_{P}=-50$, $\mathrm{u}_{\infty}=7800 \mathrm{~m} / \mathrm{s}, \mathrm{T}_{\mathrm{c}}=0.2 \mathrm{eV}, \mathrm{m}_{\mathrm{i}}=16 \mathrm{amu}$; Contour values: $\phi=-.1,-1,-10,-20,-30$ from the freestream toward the plates

26. Normalized current density $J /\left(e n_{\infty} u_{\beta}\right)$ to the inside surfaces of two equally biased flat plates for different separation distances: $\mathrm{d}_{\mathrm{p}}=5 \lambda_{\mathrm{D}}, \phi_{\mathrm{p}}=-50, \mathrm{u}_{\infty}=7800 \mathrm{~m} / \mathrm{s}, \mathrm{T}_{\mathrm{c}}=0.2 \mathrm{eV}$, $\mathrm{m}_{\mathrm{i}}=16$ amu

27. u velocity contours for the case of an electrically floating bottom plate: $d_{\text {sep }}=24 \lambda_{D}$, $\mathrm{d}_{\mathrm{p}}=5 \lambda_{\mathrm{D}}, \phi_{\mathrm{p}(\mathrm{log})}=-50, \phi_{\mathrm{f}(\mathrm{box})}=-4.3$, $\mathrm{u}_{\infty}=7800 \mathrm{~m} / \mathrm{s}, \mathrm{T}_{\mathrm{e}}=0.2 \mathrm{eV}, \mathrm{m}_{\mathrm{i}}=16 \mathrm{amu}$; Contour values: $u / u_{\infty}=1.005,1.01,1.05,1.1,1.2$, 1.6 from the freestream toward the plates

28. $v$ velocity contours for the case of an electrically floating bottom plate: $d_{s c p}=24 \lambda_{D}$, $\mathrm{d}_{\mathrm{p}}=5 \lambda_{\mathrm{D}}, \phi_{\mathrm{p}(\mathrm{cop})}=-50, \phi_{\mathrm{f}(\mathrm{boc})}=-4.3$, $u_{\infty}=7800 \mathrm{~m} / \mathrm{s}, \mathrm{T}_{\mathrm{e}}=0.2 \mathrm{eV}, \mathrm{m}_{i}=16 \mathrm{amu}$; Contour values: $v / u_{\infty}= \pm .05, \pm .1, \pm .25, \pm .5, \pm .75$ positive values below the plates, negative values above from the freestream toward the plates

29. Ionic density contours for the case of an electrically floating bottom plate: $d_{s e p}=24 \lambda_{D}$, $\mathrm{d}_{\mathrm{p}}=5 \lambda_{\mathrm{D}}, \phi_{\mathrm{p}(\mathrm{Lop})}=-50, \phi_{\mathrm{f}(\mathrm{boc})}=-4.3$, $\mathrm{u}_{\infty}=7800 \mathrm{~m} / \mathrm{s}, \mathrm{T}_{\mathrm{c}}=0.2 \mathrm{eV}, \mathrm{m}_{\mathrm{i}}=16 \mathrm{amu}$; Contour values: $n_{\mathrm{i}} / n_{\infty}=.95, .7, .5$ from the freestream toward the plates 


\section{LIST OF FIGURES (CONTINUED)}

Figure

Page

30. Electric potential contours for the case of an electrically floating bottom plate: $d_{s e p}=24 \lambda_{D}$, $d_{p}=5 \lambda_{D}, \phi_{p(\text { lop })}=-50, \phi_{f(b o c)}=-4.3$, $\mathrm{u}_{\infty}=7800 \mathrm{~m} / \mathrm{s}, \mathrm{T}_{\mathrm{e}}=0.2 \mathrm{eV}, \mathrm{m}_{\mathrm{i}}=16 \mathrm{amu}$; Contour values: $\phi=-.01,-.1,-1,-4.3,-10,-20$, from the freestream toward the plates

31. u velocity contours for the case of an electrically floating bottom plate: $d_{s c p}=9 \lambda_{D}$, $d_{p}=5 \lambda_{D}, \phi_{p(\text { lop })}=-50, \phi_{f(b o x)}=-4.6$, $\mathrm{u}_{\infty}=7800 \mathrm{~m} / \mathrm{s}, \mathrm{T}_{\mathrm{e}}=0.2 \mathrm{eV}, \mathrm{m}_{\mathrm{i}}=16 \mathrm{amu}$; Contour values: $u / u_{\infty}=1.005,1.01,1.05,1.1,1.2$, 1.6 from the freestream toward the plates

32. v velocity contours for the case of an electricaliy floating bottom plate: $d_{s e p}=9 \lambda_{D}$, $d_{p}=5 \lambda_{D}, \phi_{p(\text { top })}=-50, \phi_{f(b o c)}=-4.6$, $u_{\infty}=7800 \mathrm{~m} / \mathrm{s}, \mathrm{T}_{\mathrm{e}}=0.2 \mathrm{eV}, \mathrm{m}_{\mathrm{i}}=16 \mathrm{amu}$; Contour values: $v / u_{\infty}= \pm .05, \pm .1, \pm .25, \pm .5, \pm .75$ positive values below the plates, negative values above from the freestream toward the plates

33. Ionic density contours for the case of an electrically floating bottom plate: $d_{s e p}=9 \lambda_{D}$, $d_{p}=5 \lambda_{D}, \phi_{p(\text { top })}=-50, \phi_{f(b o c)}=-4.6$, $\mathrm{u}_{\infty}=7800 \mathrm{~m} / \mathrm{s}, \mathrm{T}_{\mathrm{e}}=0.2 \mathrm{eV}, \mathrm{m}_{\mathrm{i}}=16 \mathrm{amu}$; Contour values: $n_{i} / n_{\infty}=.95, .7, .5$ from the freestream toward the plates

34. Electric potential contours for the case of an electrically floating bottom plate: $d_{x p}=9 \lambda_{D}$, $d_{p}=5 \lambda_{D}, \phi_{p(\text { lop })}=-50, \phi_{\mathrm{f}(\mathrm{boc})}=-4.6$, $\mathrm{u}_{\infty}=7800 \mathrm{~m} / \mathrm{s}, \mathrm{T}_{\mathrm{e}}=0.2 \mathrm{eV}, \mathrm{m}_{\mathrm{i}}=16 \mathrm{amu} ;$ Contour values: $\phi=-.01,-.1,-1,-4.6,-10,-20$, from the freestream toward the plates

35. $u$ velocity contours for the case of an electrically floating bottom plate: $\alpha_{x c p}=4 \lambda_{D}$, $d_{p}=5 \lambda_{D}, \phi_{p(\text { lop })}=-50, \phi_{f(b o t)}=-4.75$, $\mathrm{u}_{\infty}=7800 \mathrm{~m} / \mathrm{s}, \mathrm{T}_{\mathrm{e}}=0.2 \mathrm{eV}, \mathrm{m}_{\mathrm{i}}=16$ amu; Contour values: $u / u_{\infty}=1.005,1.01,1.05,1.1,1.2$, 1.6 from the freestream toward the plates 
Figure

Page

36. v velocity contours for the case of an electrically floating bottom plate: $\mathrm{d}_{x \mathrm{p}}=4 \lambda_{\mathrm{D}}$, $\mathrm{d}_{\mathrm{p}}=5 \lambda_{\mathrm{D}}, \phi_{\mathrm{p}(\mathrm{lop})}=-50, \phi_{\mathrm{f}(\mathrm{boc})}=-4.75$, $\mathrm{u}_{\infty}=7800 \mathrm{~m} / \mathrm{s}, \mathrm{T}_{\mathrm{e}}=0.2 \mathrm{eV}, \mathrm{m}_{1}=16$ amu; Contour values: $v / u_{\infty}= \pm .05, \pm .1, \pm .25, \pm .5, \pm .75, \pm 1$ positive values below the plates, negative values above from the freestream toward the plates

37. Ionic density contours for the case of an electrically floating bottom plate: $d_{s e p}=4 \lambda_{D}$, $d_{p}=5 \lambda_{D}, \phi_{p(\text { top })}=-50, \phi_{\mathrm{f}(\mathrm{bot})}=-4.75$, $u_{\infty}=7800 \mathrm{~m} / \mathrm{s}, \mathrm{T}_{\mathrm{c}}=0.2 \mathrm{eV}, \mathrm{m}_{\mathrm{i}}=16 \mathrm{amu}$; Contour values: $n_{\mathrm{i}} / \mathrm{n}_{\infty}=.95, .7, .5, .3, .1$

38. Electric potential contours for the case of an electrically floating bottom plate: $d_{s e p}=4 \lambda_{D}$, $d_{p}=5 \lambda_{D}, \phi_{p(\text { cop })}=-50, \phi_{\text {f(bor })}=-4.75$, $\mathrm{u}_{\infty}=7800 \mathrm{~m} / \mathrm{s}, \mathrm{T}_{\mathrm{e}}=0.2 \mathrm{eV}, \mathrm{m}_{\mathrm{i}}=16 \mathrm{amu}$; Contour values: $\phi=-.01,-.1,-1,-4.75,-10,-20$, from the freestream toward the plates

39. Floating potential of the bottom plate as a function of separation distance: $d_{p}=5 \lambda_{D}$, $\phi_{p(L p)}=-50, u_{\infty}=7800 \mathrm{~m} / \mathrm{s}, T_{c}=0.2 \mathrm{eV}, m_{i}=16 \mathrm{amu}$ 


\section{LIST OF TABLES}

Table

Page

I. Comparison between analytical and computed electric sheath parameters for a single semiinfinite flat plate: $u_{\infty}=8780 \mathrm{~m} / \mathrm{s}, T_{e}=0.2 \mathrm{eV}$, $\phi_{\mathrm{p}}=-20, \mathrm{~m}_{\mathrm{i}}=16$

II. Comparison between analytical and computed electric sheath parameters for a single semiinfinite flat plate: $u_{\infty}=8780 \mathrm{~m} / \mathrm{s}, T_{e}=0.2 \mathrm{eV}$, $\phi_{p}=-10, m_{i}=16$

III. Floating potential calculation as a function of orbital altitude for a single finite flat plate 
CHAPTER I

INTRODUCTION

The term plasma is used to describe a large class of basically neutral mixtures containing some electrically charged particles. Because some of the particles are charged they interact with each other in accordance with the coulombforce law. The coulomb-force is relatively long range. Hence, in a plasma every charged particle interacts simultaneously with many of its neighbors, giving the plasma a cohesiveness [ref.1]. Another related feature of a plasma is that the charged particles tend to rearrange themselves in such a way as to effectively shield any electrostatic fields due either to a surface at some nonzero electric potential or to a region of net charge density within the plasma. This phenomena is due to the fact that particles with like charge tend to repel while those of opposite charge attract, thereby neutralizing charge concentrations.

In low earth orbit (LEO), the ambient plasma is composed of neutral particles, electrons and various ionic species [ref.2]. The neutral and ionic species are as much as $10^{4}$ 
times as massive as the electrons. Therefore, at a given temperature, the electron thermal velocity is orders of magnitude greater than that of the ions and neutrals. At an altitude of $200 \mathrm{~km}$, for example, an orbiting spacecraft has a velocity of around $7800 \mathrm{~m} / \mathrm{s}$ which is greater than that of the thermal ions but much slower than the thermal electrons. Such a flow is referred to as mesothermal. The plasma is also only slightly ionized. The flow field of the neutral particles is therefore not affected by the presence of the ionized species.

When a spacecraft is immersed in the low earth orbit plasma, its surfaces will tend to charge slightly negative in response to the greater mobility of the electrons relative to the heavy positive ionic species. As the spacecraft charges, the negative electric field begins to repel the electrons while attracting the positive ions. Charging continues until the positive ions can be attracted at a rate (i.e., electric current) equal to that of the more mobile electrons. At this point, the spacecraft has reached what is called the 'floating' potential. The plasma in proximity to the surfaces will be redistributed in such a way as to shield the bulk of the ambient plasma from the resulting electrostatic field. The result will be a slightly negatively charged spacecraft surrounded by a cloud of net positive charge. The disturbed region where the shielding takes place is called the sheath with dimensions that depend on the magnitude of the electrostatic field, the properties of the plasma and the properties of the spacecraft surface. The spacecraft thus 
imposes an abrupt boundary condition which is relaxed within a finite distance through the sheath into the bulk of the ambient plasma. The sheath is seen as the medium through which the spacecraft interacts with its environment, and knowledge of its structure is important in modeling a range of phenomena.

The interaction is further complicated by the fact that a typical spacecraft is composed of several materials, each interacting differently with the space plasma. Internally imposed electric potentials may also be present such as that from an exposed solar array. The interconnection of the surfaces to the spacecraft and to each other, and how the spacecraft is connected to the power system determines how each surface will charge in response to the plasma. Overall, the net current to the entire spacecraft will be zero in order for equilibrium to be established. However, this does not necessarily mean that the net current to each surface is zero, only that the total sum of current to all surfaces is zero. Therefore, in general, a surface will be charged either positive or negative relative to the floating potential.

The magnitude of the current to a surface as a function of its properties, electric potential, proximity to other surfaces, and the properties of the space plasma, has been the object of many past studies. The present study focuses on how two surfaces in close proximity will interact in the space environment. The geometry shown in Figure 1 is that of two parallel finite flat plates, biased to different electric 


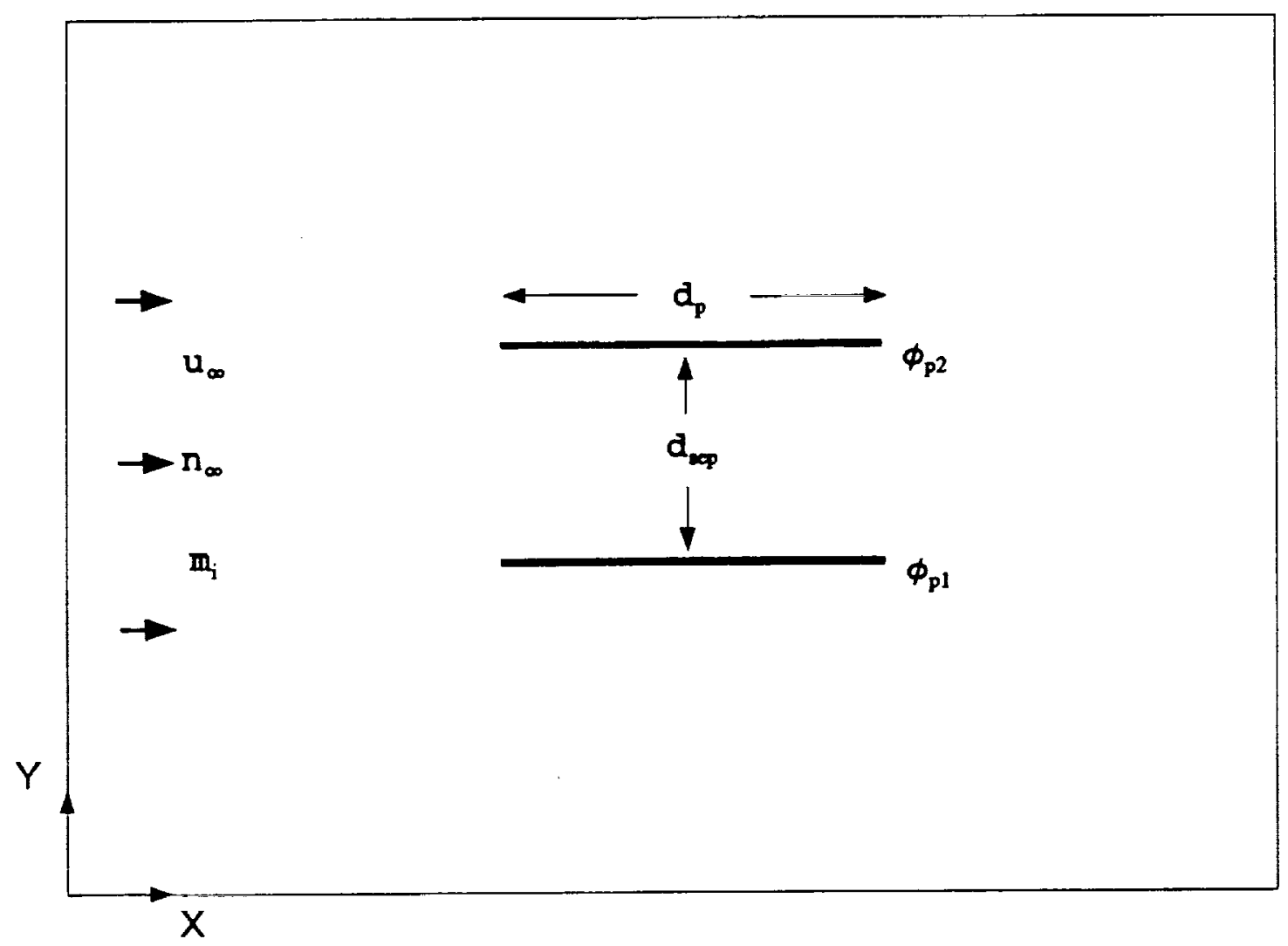

Figure 1. Schematic of a two-plate geometry 
potentials, oriented parallel to a flowing plasma characteristic of LEO. For geometries other than the most symmetric, computational methods must be employed to model the interaction because analytical methods are impractical.

There are two main areas of study which consider the interaction of a spacecraft with its environment; classical probe theory and ionospheric aerodynamics. In Chapter II, the approach of each will be outlined and a review of past investigations given.

In Chapter III, the characteristics of the interaction are identified, the governing equations are summarized, and the numerical approach employed is described.

In Chapter IV, results from the three case studies are presented. First, the flow field about a single semi-infinite flat plate is compared to an analytical and a computational approach proposed in the literature. Next, the interaction of two equally biased plates is studied. Results are presented which describe how the current collected by each plate is affected by separation distance and applied potential. As a final application, the docking of two spacecraft in orbit (i.e. two parallel flat plates moving toward one another) will be studied in a quasi-static manner.

Chapter $V$ summarizes the major conclusions and areas of future work are discussed. 
CHAPTER II

BACKGROUND

The approach used to model the interaction of a spacecraft with its environment depends on which phenomena are being studied, and on the properties (i.e. especially the density) of the plasma. Whether it is decided to apply a continuum or free-molecular model to the problem usually depends on the knudsen number which is the ratio of the effective plasma mean free path to the dimensions of the satellite. If the Knudsen number is less than 0.01 , then the macroscopic continuum equations can be used. otherwise, a free-molecular approach should be applied. For altitudes below about $200 \mathrm{~km}$, the dimensions of a spacecraft are likely to be of the same order of magnitude or greater than the mean free path [ref. 3].

The two mathematical approaches most often used are the kinetic and the phenomenological. Using kinetic theory, a statistical approach for studying the interaction is developed, where it is recognized that the macroscopic properties of the plasma are due to the average behavior of many individual particles, but where each individual 
particle's motion is governed by the laws of mechanics. For a system containing $\mathrm{N}$ particles, a complete description of the particles would require a specification of the positions and velocities of each particle; but to obtain a statistical description of the plasma, only a knowledge of the distribution function of the molecular velocities of the particles is required [ref.1]. The time variation of the distribution function is described by the Boltzmann transport equation which is a seven-dimensional equation and does not lend itself to analytical solution. The kinetic approach can be used to describe both free-molecular flows, where no collisional-collective behavior occurs, and a true continuum. Numerically speaking however, the kinetic approach is more difficult than the phenomenological.

In the phenomenological approach, relations between stress and rate of strain, and between heat flux and temperature gradient are postulated, and the continuum fluid equations are then developed from conservation laws. The resulting system consists of a subset of the general Navierstokes equations with the addition of electrical terms, and Maxwell's electromagnetic equations. The fluid equations can also be obtained by taking moments of the Boltzmann equations, provided that the appropriate assumptions are made [ref.4]. The use of the fluid equations is usually restricted to high density, collision dominated flows. However, the continuum approach may be valid in modeling a 'collisionless' plasma because a collective behavior may occur as a result of the 
relatively long range electrostatic forces. The literature review to follow focuses on articles based on the continuum fluid model in the areas of continuum probe theory and ionospheric aerodynamics.

Continuum Probe Theory

Probes have long been used as a diagnostic tool for measuring the properties of a plasma. Continuum probe theory utilizes the continuum concepts of diffusion and mobility to derive relations which enable the properties of the undisturbed plasma to be approximated from the current-voltage characteristics of a probe.

Electric probe theory was pioneered by Langmuir in 1924 . The Langmuir probe is still widely used as a diagnostic tool for measuring the local properties of plasmas. A useful summary of the available theoretical results on the use and behavior of electric probes was given by Chen [ref. 5]. More recently, a literature survey on electric probes was written by chung, et. al. [ref. 6] for collisionless, transitional, and continuum probes. The classifications used in the second part of the Chung, et.al. paper entitled "Continuum Probes" are based on the relative importance of convection, diffusion, and mobility on the motion of the charge carriers; electrons and heavy positive ions. These effects can be better seen by recasting the governing equations in terms of flux density, and nondimensionalizing.

The system of governing equations consists of ion and 
electron species continuity equations, conservation of momentum and energy equations, and Poisson's equation which governs the electric field assuming a negligible magnetic field. The continuity equations can be recast in terms of flux density defined as $\Gamma_{\alpha}=n_{\alpha} v_{\alpha}$. Another equation for the flux density can be obtained from the momentum equation, with the result being [ref. 7]

$$
\Gamma_{\alpha}=-D_{\alpha} \nabla n_{\alpha}+n_{\alpha} \mu_{\alpha} E
$$

where $D_{\alpha}$ is the free diffusion coefficient defined by

$$
D_{\alpha}=\frac{k T_{\alpha}}{m_{\alpha} v_{\alpha}}
$$

and $\mu_{\alpha}$ is the mobility defined by

$$
\mu_{\alpha}=\frac{g_{\alpha}}{m_{\alpha} v_{\alpha}}
$$

The Einstein relation relates the two parameters

$$
\frac{D_{\alpha}}{\mu_{\alpha}}=\frac{k T_{\alpha}}{g_{\alpha}}
$$

From equation (2.1), flux density $\Gamma$ is seen to be composed of diffusion of the charged particles by means of a density gradient (i.e. collisional process) and by the mobility of the charged particles by means of an electric field (i.e. electrical process). Combining the flux density and species continuity equations, and nondimensionalizing, results in an equation containing convection, diffusion, and mobility terms [ref, 8,9$]$. 


$$
R_{e} S_{c_{1}} \rho \frac{d}{d t}\left(\frac{n_{\alpha}}{\rho}\right)-\nabla \cdot\left(\mu_{\alpha} D_{\alpha}\left[\nabla \frac{n_{\alpha}}{\rho} \pm \frac{1}{T} \frac{n_{\alpha}}{\rho} \nabla \phi\right]\right)=0
$$

where the plus sign is for positive ions and the minus sign for electrons. Poisson's equation in non-dimensional form is given by

$$
\left(\frac{\lambda_{D}}{R_{0}}\right)^{2} \nabla^{2} \phi=-\left(n_{i}-n_{e}\right)
$$

The conservation of momentum and energy equations which include electric body forces and electric (i.e. Joule) heating round out the system.

The two most important parameters are seen to be $R_{c} S_{c i}$ which weighs the importance of the viscous layer and convective effects (i.e. Prandtl boundary layer) and $\lambda_{D} / R_{0}$ which is a measure of the electrical disturbance caused by the probe (i.e. Langmuir boundary layer). The relative values of $\lambda_{D} / R_{0}$ and $R_{c} S_{c i}$ also determines the extent of the electrical disturbance. For example, if the following inequalities are satisfied, [ref. 7]

$$
\left(\frac{\lambda_{D}}{R_{0}}\right)^{2} \ll \frac{1}{R_{e}}<1
$$

then the electrical boundary-layer is imbedded within the viscous layer.

The different regimes of continuum probe operation can thus be seen to be divided between the quiescent $R_{c} S_{c i} \rightarrow 0$, and convection dominated (i.e. flowing) $R_{e} S_{c i} \rightarrow \infty$, and further 
subdivided as to the extent of the electrical disturbance.

The goal of studies in probe theory is to obtain a theoretical or an attainable numerical current-voltage characteristic curve for the probe, and the equations which describe the relationship between the current and the local plasma properties for different portions of the curve. An experimenter can then measure the current to the probe for different bias voltages, obtain a current-voltage curve and from the shape of the curve determine the local plasma properties (i.e. density and temperature). The problem is often simplified by postulating regions where different physical mechanisms dominate, and solving the resulting equations accordingly.

In the small convection limit, pioneering papers were written by su and Lam [ref. 10], and Cohen [ref. 11] for thin sheath, frozen chemistry, constant property plasma. The governing equations in this case reduce to the flux-continuity equation and Poisson's equation. For symmetric probe geometry, the interaction becomes one-dimensional. In both papers, an inner sheath region and an outer quasi-neutral region were postulated, and the equations solved separately in each region based on those assumptions. The two solutions were matched by asymptotic expansions.

In the large convection limit, a directional flow is superimposed upon the diffusion and mobility process and the interaction becomes two-dimensional. Although intrinsically more complicated than the quiescent case, an assumption was 
often made to simplify the analysis - a thin sheath was assumed so that convective effects in the sheath could be neglected, and the interaction in the sheath becomes again one-dimensional. Lam [ref. 12] pioneered the work in this area for an incompressible, constant property plasma flow about an arbitrary solid body. It was found in this and further work that the flow field naturally divided up into three or more regions; the inviscid region where convection dominates, the viscous layer where both convection and diffusion processes occur, and the sheath in which convection is neglected compared to diffusion and mobility [ref. 8,9]. It should also be noted that as the bias voltage of the probe becomes more negative, the influence of convection decreases and that of diffusion and mobility increases. This point was used by stahl and su [ref. 13], and subsequently by Giles, et.al. [ref. 14], to make it possible to ignore convection even when the sheath region approaches the thickness of the viscous layer.

In the case when a thick sheath is dealt with explicitly, Poisson's equation becomes two-dimensional and convective effects in the sheath are important. Johnson and Deboer [ref. 15] included the convection effects for high speed plasma flow over flat-plate and cylindrical probes aligned parallel with the flow. Russo and Touryan [ref. 16] gave a rather complete analysis including finite chemical reactions at a range of probe potentials.

In order for current to flow, two electrodes must be in 
contact with the plasma. In electrostatic probe theory, the simplest technique is to use a single probe and to assume that the second conceptualized electrode is grounded at the undisturbed plasma potential. Chung and Blankenship [ref. 17] explained that this approximation is not always reliable and developed the theory of an electrostatic double probe consisting of two finite parallel plates. The flow field was divided into different physical regimes, and the governing equations solved accordingly. In the development, it is assumed that the sheaths of the two plates do not interact directly but are separated by a quasi-neutral, inviscid region. The mechanism by which electricity is conducted between the two plates through the flowing plasma is discussed.

Just recently, vitello, et.al. [ref. 18] described a twodimensional time-dependent numerical fluid code FLOW simulating ion extraction from a plasma. The electrons were assumed to be in equilibrium but no approximation was used for the ions. The fluid equations were solved for the ions using finite differences through the entire flow region. This approach closely resembles the present one, but the application and flow geometry differs.

\section{Ionospheric Aerodynamics}

Ionospheric aerodynamics is concerned with the effects that the presence of an artificial satellite has on the ambient space plasma. Although a spacecraft is in essence a 
probe, the emphasis is on the behavior of the disturbed plasma flow field around the body, and not on determining plasma properties.

The set of equations which govern the continuum flow of a plasma over or around a satellite with electric body forces present consist of conservation equations for charge, mass, momentum and energy, equations of state and Poisson's equation. The plasma is treated as an interacting mixture of electrons, positive ions, and neutral particles. Multiple sets of continuum equations must be solved for each species, with interaction terms present in each [ref. 1]. Typically there are several assumptions made about the interaction which simplify the system of equations.

First, the satellite velocity is intermediate between the ion thermal speed and the electron thermal speed. This simplifies the treatment of the electrons. The electrons can be considered to be in local thermodynamic equilibrium. For the case of highly negative surface potentials $\left(|\phi|>k \mathrm{~T}_{\mathrm{e}} / e\right)$ under steady-state conditions, this allows the use of the Boltzmann factor in determining the electron density as a function of electric potential. The Boltzmann factor provides all the necessary information about the electrons.

The momentum transfer to and from different species is considered for ionospheric conditions to be dominated by the external electrostatic body force and pressure force [ref. 1]. If desired, transfer of momentum by discrete collisions can be expressed by an interaction term which can take on various 
functional forms dependent on relative velocities, masses, etc.. To simplify the analysis further, the plasma is assumed 'collisionless' in the sense that the momentum transfer through discrete collisions is negligible [ref. 19]. The interaction term is often dropped from the analysis.

Since the velocity of the ions is much less than that of the electrons, the cold-ion approximation is often employed. As a result, the ions make negligible contribution to the pressure and interaction terms in the momentum equation [ref. 4]. The trajectories of the ions are therefore identified with macroscopic streamlines and the continuity and momentum equations represent 'free-stream' ions.[ref. 20]. The energy equation for ions is no longer needed.

After applying the above assumptions, the starting point of most past studies employing the continuum fluid approach are the following equations:

ion continuity

$$
\frac{\partial n_{i}}{\partial t}+\nabla \cdot\left(n_{i} v_{1}\right)=0
$$

ion momentum

$$
n_{i} m_{i} \frac{d \boldsymbol{v}}{d t}=-\nabla p+e n_{i} \boldsymbol{E}
$$

electrostatic potential

$$
\boldsymbol{E}=-\nabla \boldsymbol{\phi}
$$


Poisson's equation

$$
\nabla \cdot \boldsymbol{B}=-\frac{e}{\epsilon_{0}}\left[n_{i}-n_{e}\right]
$$

equation of state

$$
\frac{d}{d t}\left(p n_{i}^{-\gamma}\right)=0
$$

Boltzmann factor

$$
n_{e}=n_{\infty} e^{\frac{\phi \phi}{k T_{e}}}
$$

Another important parameter is the Debye length defined by

$$
\lambda_{D}=\left(\frac{e_{0} k T_{e}}{e^{2} \Pi_{\infty}}\right)^{1 / 2}
$$

The Debye length defines an order of magnitude distance in which electrostatic fields are shielded from the bulk of a plasma.

The bulk of the literature in this area is from the late 50 's to the early 70's. Due to the complexity of the interaction, it was impossible for computers of the time to solve the general system of equations in a reasonable amount of time. In order to make the problem solvable, regions where different physical mechanisms dominated were postulated, and the equations simplified based on those assumptions.

Lam and Greenblatt [ref. 21] considered the flow of a collisionless plasma about a highly negatively biased cone with the characteristic body length $R_{0}$ much greater than the 
Debye length. The flow field was divided into a quasi-neutral $\left(n_{i}=n_{e}\right)$ and sheath region $\left(n_{i}>n_{e}\right)$. since $R_{o} \gg \lambda_{D}$, the sheath was assumed to be very thin, and could be approximated by the body surface. This was used to uncouple the sheath from the solution of the quasi-neutral region except at their common boundary. Lam and Greenblatt showed that under these conditions in the quasi-neutral region the ionic velocity was irrotational. A velocity potential can thus be defined and a potential equation derived which is analogous to the potential equation for conventional isothermal compressible flow with the ratio of the kinetic energy of the ions to the thermal energy of the electrons playing the role of the square of the Mach number [ref. 22]. Whether this parameter was greater or less than one determined the characteristics of the flow. If the flow is supersonic, there will be no upstream signal propagation.

The technique used by Lam and Greenblatt was to simplify the equations by making further assumptions about the ionic density. In the quasi-neutral region, Poisson's equation no longer needed to be solved, which allowed the definition of a velocity potential. Various other approximations have been used for the ionic density in the literature.

For example, Hohl and wood [ref. 23] studied the processes that are involved in the electric drag forces on a large spherical satellite at its floating potential. The floating potential of a sphere is only slightly negative due to the greater electron thermal velocity. For such 
potentials, the ion number density in the sheath was approximated to the first order by Hohl and wood to be constant and equal to that outside the sheath. The electron number density was determined using a modified Boltzmann distribution. Hohl and wood used a computational method similar to that of Davis and Harris [ref. 24] which involved iterating between tracking ion trajectories to find the ionic density and solving Poisson's equation for the potential.

Several of the sources referenced thus far in this section [ref. 1- 4, 20] are general review articles on ionospheric aerodynamics. The article by stone [ref. 4] attempts to integrate information existing at that time into a parametric treatment of the problem. The different possible formulations (i.e. continuum and kinetic) are outlined quite well, and a review of the results from a range of studies is given.

More recently, Wang and Hastings [ref. 25] studied the dynamic coupling of large highly negatively biased flat plates with a flowing plasma on the ion-plasma-time scale. A particle simulation method was used to obtain the density profile of the ions for various time-dependent boundary conditions. Steady state results were also presented. 
CHAPTER III

APPROACH AND FORMULATION

The interaction of two parallel flat plates biased to different electric potentials in close proximity immersed in a flowing plasma is studied numerically. The following assumptions are based on the characteristics of the interaction between an orbiting spacecraft and the low earth orbit plasma.

\section{Assumptions}

1). The speed of the spacecraft is intermediate between the thermal velocities of the ions and electrons.

$$
V_{T_{1}}<U_{\infty}<V_{T_{*}}
$$

The electrons are therefore assumed to be in equilibrium, and are unaffected by the motion of the spacecraft. The electron number density as a function of the local potential is obtained. from the solution of the collisionless Boltzmann equation known as the Boltzmann factor

$$
n_{e}=n_{\infty} e^{\frac{\phi \phi}{k T_{e}}}
$$


The Boltzmann factor provides all information needed about the electronic behavior. The ions are considered to have negligible thermal motion; an energy equation for the ions is therefore not necessary.

2). The speed of the oncoming ions is assumed to be great enough to satisfy Bohm's criteria

$$
v_{i}^{s} \geq u_{\beta}=\sqrt{\frac{k T_{e}}{m_{i}}}
$$

An electric sheath thus forms around imposed electric potentials on the flat plates causing the potential to be shielded from the bulk of the plasma on the order of a Debye length away from the surface. Boundary conditions can thus be established at infinity as those in an undisturbed plasma; $\nabla=u_{\infty}, n_{c}=n_{i}=n_{\infty}$. The Debye length is defined as

$$
\lambda_{D}=\left(\frac{\epsilon_{0} k T_{\theta}}{e^{2} n_{\infty}}\right)^{1 / 2}
$$

The Debye length is typically on the order of a centimeter for the LEO plasma

3). The plates are assumed to be perfect absorbers of incident particles, are of infinitesimal thickness, with surface potential $\phi_{p}$. All ions reaching the plate are assumed neutralized and do not contribute to the electric potential on the plate unless the plate is specified as 
'floating'.

4). The plasma is only slightly ionized. The flow field of the neutral particles is therefore not affected by the presence of the charged particles, and can be determined by the usual means.

5). The plasma is assumed collisionless in the sense that the charged particles only interact through their contribution to the electric potential. The gradient in the electric potential determines the body forces.

6). The interaction is modeled in steady state. When studying the application of the docking of two spacecraft, the problem is modeled in a quasi-static manner which assumes that the electric sheath structure of the two plates establishes itself on a time scale much faster than the movement of the plates.

\section{Governing Equations}

Based on the assumptions outlined above, the system of equations governing the ions is a subset of the general Navier-Stokes equations with the addition of electric body force terms. Poisson's equation governs the electric potential, and the Boltzmann factor provides the electronic number density.

In non-dimensional form, the equations for steady-state, 
compressible, laminar flow are

Ion continuity

$$
\frac{\partial\left(n_{i} u\right)}{\partial x}+\frac{\partial\left(n_{i} v\right)}{\partial y}=0
$$

Ion $\mathrm{x}$-momentum

$$
u \frac{\partial u}{\partial x}+v \frac{\partial u}{\partial y}=-\frac{1}{M_{0}^{2}} \frac{\partial \phi}{\partial x}
$$

Ion $\mathrm{y}$-momentum

$$
u \frac{\partial v}{\partial x}+v \frac{\partial v}{\partial y}=-\frac{1}{M_{0}^{2}} \frac{\partial \phi}{\partial y}
$$

Poisson's

$$
\frac{\partial^{2} \phi}{\partial x^{2}}+\frac{\partial^{2} \phi}{\partial y^{2}}=n_{e}-n_{i}
$$

Boltzmann factor

$$
n_{e}=e^{\phi}
$$

Ambient Mach number

$$
M_{0}=\frac{u_{\infty}}{\sqrt{\frac{k T_{e}}{m_{i}}}}
$$

The velocity components and the density of the ions and electrons were normalized by the free-stream values $u_{\infty}$ and $n_{\infty}$ respectively. The spatial components were normalized by the Debye length, and the potential by the thermal energy of the electrons.

The ambient Mach number is defined as the ratio of the 
freestream or orbital velocity to the ion sound speed. Both the orbital velocity and the ion sound speed are functions of the orbital altitude. As one increases in altitude, the orbital velocity and the ion mass decrease while the electron temperature increases. The ion sound speed increases more rapidly than the orbital velocity decreases resulting in a decrease in the ambient Mach number with increasing altitude.

\section{Computational Approach}

The equations are solved using finite-differences with the frame of reference fixed on the plates. A staggered and variable grid is employed. A separate grid is defined for each velocity component and one for the potential and density. The velocity components are located midway between the potential and density points. The potential gradient, which determines the body force on the ions, thus becomes the 'natural driving force' for the velocity components [ref. 26]. A variable grid was employed in order to cluster grids in regions of expected large flow gradients; the leading and downstream edges of the plates, and along the surfaces of the plates. Simple geometric clustering equations were used. The governing equations were not transformed in terms of the clustering equations however. Instead, the governing equations are cast into a general, variable-grid finitedifference form [ref. 27]. Figure (2) shows the variable grid structure used for a two-plate geometry. The plasma flows from left to right in the $x$-direction. 


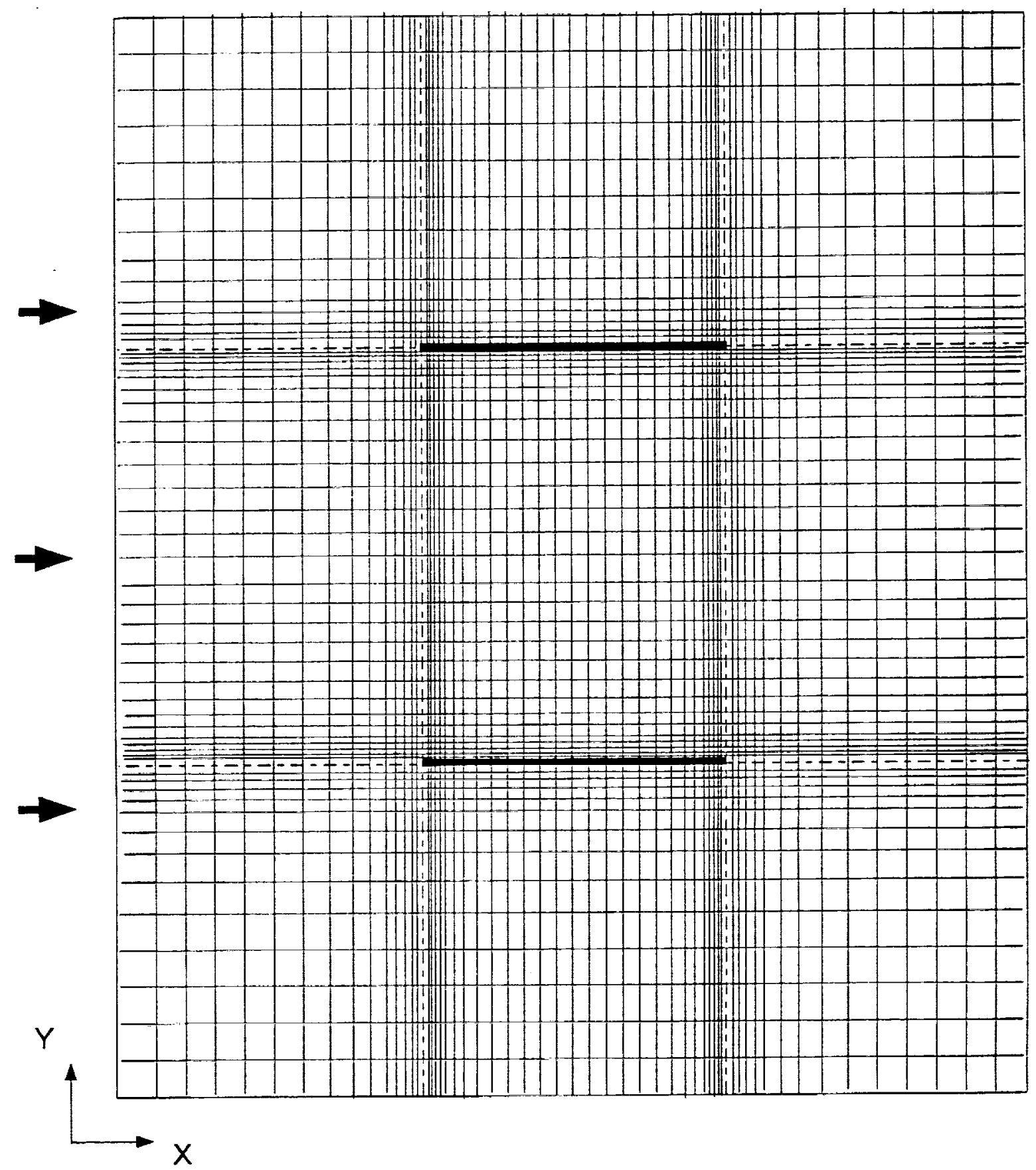

Figure 2. Variable grid structure for a two-plate geometry 
Poisson's equation is elliptic in nature, and is differenced using a five-point formula. The equation is made linear by using the potential determined in the previous iteration in the Boltzmann factor. The resulting system of linear equations is solved using the Thomas Algorithm with successive over-relaxation (SOR) by columns.

An explicit second-order upwind differencing scheme is used for the $u$ velocity, and an explicit first-order upwind scheme is used for the $\mathrm{v}$ velocity. A second order scheme was not chosen for the $v$ velocity because of the numerous regions of reversed flow expected in the cross-flow direction for the problems studied. No flow-reversal is expected in the $x-$ direction. The equations are made linear by either lagging or extrapolating the coefficients of the convective terms. The potential gradients in the momentum equations are forward differenced.

The continuity equation is solved using a flux-balance method. A balance is applied to each control volume centered on the density grid points. The density which satisfies the continuity equation given the velocities at each face of the control volume is then determined.

The variable-grid forms of the upwind differencing schemes used are not conservative for a general case. The extent of error thereby introduced is determined by comparing the influx of momentum at the upstream boundary to the outflux of momentum at the plates and the downstream boundary. It was found that the difference was less than $5 \%$ for all case 
studies.

To obtain a steady-state solution, it is necessary to iterate between the solution of Poisson's equation and the continuum equations. Poisson's equation is solved first assuming a constant ionic and electronic number density throughout the computational space. The velocity components are then determined from the solution of the momentum equations. Finally, the continuity equation is solved to determine the ionic density which is used in Poisson's equation to adjust the potential field. This procedure is continued until there is no further change in the flow field. It was found that a complete solution of Poisson's equation at each iteration was not necessary for the solution to converge. Twenty poisson cycles at each iteration was found to be sufficient.

The inverse of the Mach number squared is present in both momentum equations and determines the extent of influence of the potential field. By decreasing the value of the Mach number, large gradients in the potential produce corresponding large changes in the velocity components which can result in the solution numerically diverging. For cases where the coefficient is relatively large, the value is increased in steps between iterations. Since only a steady-state solution is desired, this procedure is valid. If a time-dependent solution where desired, very small time-steps and/or grid spacing would be required instead.

Grid spacing is in terms of the Debye length $\lambda_{D}$. In the 
variable grid regions, spacings on the order of $.001 \lambda_{D}$ are used to begin with. In the constant grid regions, spacings on the order of . $1 \lambda_{D}$ are utilized. Grid spacings as large as $.25 \lambda_{D}$ were used successfully except for oscillations near the boundaries which is a property of the second-order upwind differencing scheme chosen.

\section{Boundary Conditions}

For Poisson's equation, boundary conditions must be defined on all four boundaries. The upstream and parallel boundaries to the flow are set far enough away from the plates that the imposed electric potentials are completely shielded prior to their position (i.e. $\phi=0$ ). The downstream boundary is approximated by

$$
\phi_{B}=\phi_{B-2}+\left[\phi_{B-1}-\phi_{B-2}\right] \frac{\left(x_{B}-x_{B-2}\right)}{\left(x_{B-1}-x_{B-2}\right)}
$$

which maintains the gradient of the potential at that point. The potential on the surface of a plate is a constant given by $\phi_{p}$, but may vary between quasi-static states if the surface is specified as 'floating'.

For the velocity components and density, the upstream and parallel boundaries are defined as free-stream; $n_{i}=n_{e}=n_{\infty}, v=0$, $\mathrm{u}=\mathrm{u}_{\infty}$. The velocity components on the downstream boundary are extrapolated from the two previous grid points.

A 'slip' boundary condition is prescribed for the $x$ component of the velocity along the surfaces of the plates. 
For the $\mathrm{y}$-component, a plate surface represents a sink which absorbs all perpendicularly incident ions. Since an upwind scheme was employed, the differencing progresses in the direction of the flow where the flow properties of each region are determined exclusively by those which are upstream or 'upwind'. The upwind direction in this case is either in the plus or minus $y$-direction depending on the direction of the acceleration by the electric body forces. Since viscosity is ignored in the present study, the velocity perpendicular to the plate does not tend toward zero as one approaches the plate. On the contrary, the accelerating force is greatest in this region. The ions are not affected by the 'physical' presence of the plate until they are actually absorbed; there is no upwind transfer of information about the velocity, except by the dependence of the potential on the gradients of the velocity components through the continuity equation.

Likewise, since the plates represent a sink, no flow can originate on the surface and move away. A situation can occur where one of the plates is at a much larger negative potential than the other, and will draw the ions away from the more positive plate. In this case, a zero $y$-component velocity boundary condition is prescribed on the plate surface.

For the situation just described, it is possible that regions of zero density may develop as momentum flows out of a region and is not replaced. This would violate the assumption of a continuum. In the present study, these situations are avoided. 
Thus far there has been little mention of what happens in the wake of a spacecraft. It is expected that if one were stationary with the plasma, that after a spacecraft passes the plasma would tend to return to an undisturbed state. The distance behind a spacecraft at which this occurs is difficult to determine [ref. 4]. In the wake, properties such as temperature and viscosity that were neglected in the region of high potential near the spacecraft now become important. The present study neglects such effects and is therefore incapable of providing a correct solution in this region. The proper way to specify the downstream boundary using the present method therefore becomes an issue especially in the case of the electric potential.

Take for example the problem of a negatively charged single plate parallel to an oncoming plasma flow. The ions are focused toward the axis of the plate on both sides. If the plate were semi-infinite, all ions entering the sheath would eventually be absorbed by the plate. If, however, the plate is of finite length, some of the focused ions will overshoot the plate causing a buildup of density behind the plate on its axis of symmetry. The buildup of positive ions can become large enough to form regions of positive potential. The plasma begins to oscillate, and a very complicated structure is produced. Even the most general continuum approach including temperature effects and viscous dissipation would not be totally sufficient because the focusing of the ions causes important behavior not represented by the average 
30

[ref. 18].

Nevertheless, the solution was carried out in the wake region using the present method to determine the effect of the placement of the downstream boundary. A complicated but structured flow pattern resulted with alternating regions of positive and negative potential. It was found that with the boundary condition of a continuous potential gradient, a distance of ten Debye lengths downstream from the back edge of the plates was sufficient to produce grid-independent results. 
CHAPTER IV

RESULTS AND DISCUSSION

\section{Single Flat plate}

The plasma flow field about a parallel flat plate can naturally be divided into regions where different physical mechanisms dominate. This technique was used by past investigators to make the problem solvable analytically. For the present application, the structure of the flow field is determined by a balance between strong convective effects in the flow direction and strong electric effects in the vertical direction. A better understanding of the processes involved is gained by analyzing the flow in more detail. Figures 3 through 8 show a flow field about a single semi-infinite flat plate for a typical set of parameters characteristic of LEO.

If there were no flow, the sheath would have a fairly constant dimension across the plate except near the very edges. When a flow is imposed, the sheath is compressed near the leading edge in the direction of the flow, and the distortion can extend many sheath thicknesses downstream. This region is labeled as the 'leading edge' in Figure 3 . The 


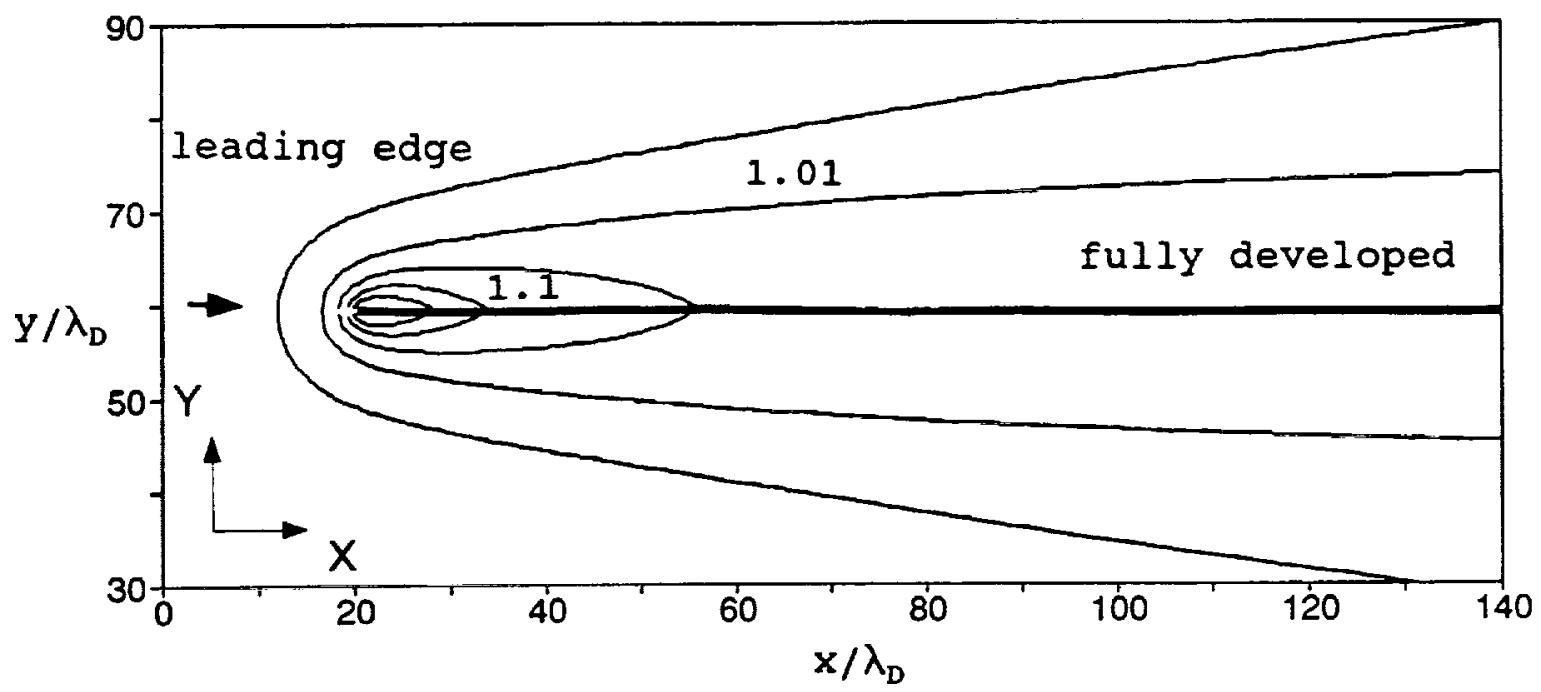

Figure 3. u velocity contours for a single semi-infinite flat plate: $u_{\infty}=8780 \mathrm{~m} / \mathrm{s}, \mathrm{T}_{\mathrm{e}}=0.2 \mathrm{eV}, \phi_{\mathrm{p}}=-20, \mathrm{~m}_{\mathrm{i}}=16 \mathrm{amu}$

Contour values: $u / u_{\infty}=1.0001,1.01,1.05,1.1,1.15$ from the freestream toward the plate

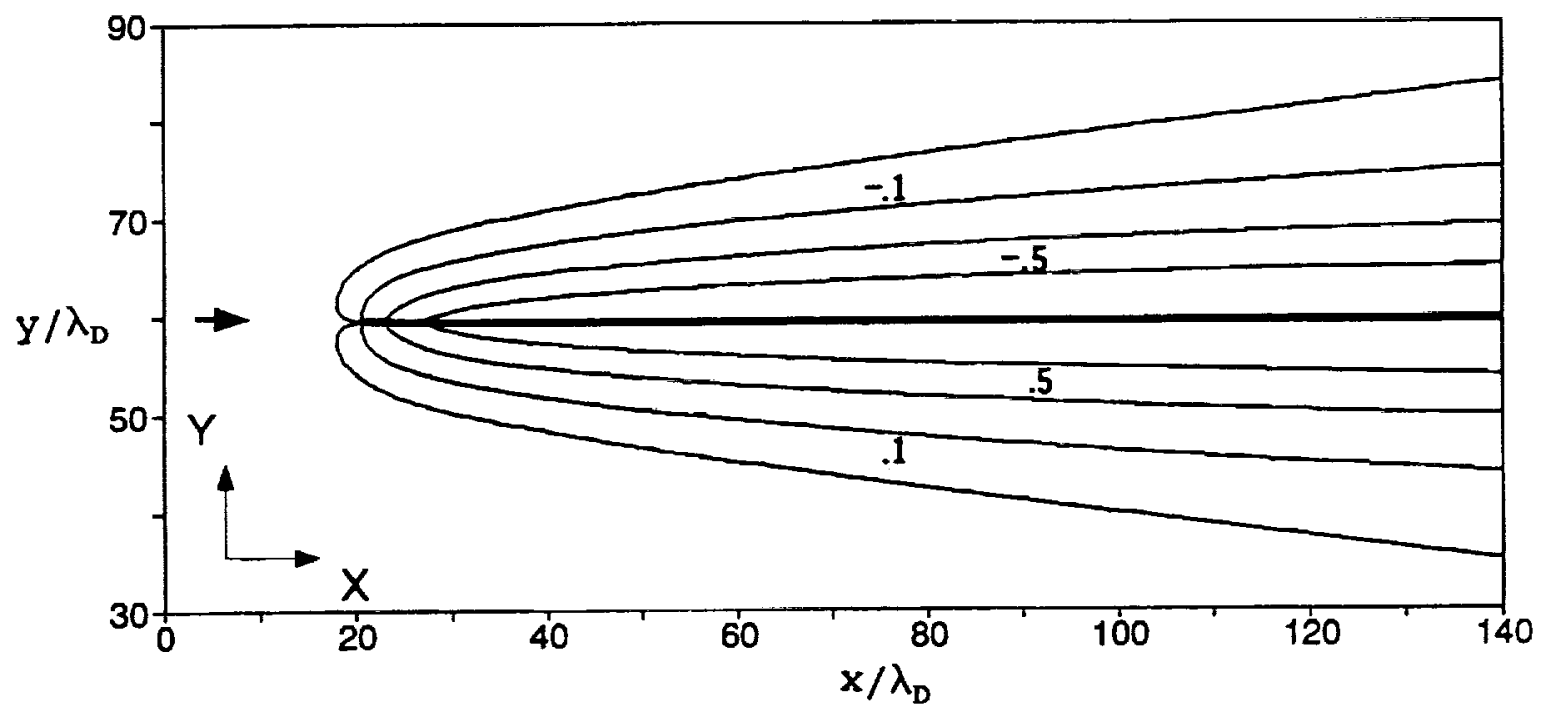

Figure 4. v velocity contours for a single semi-infinite flat plate: $u_{\infty}=8780 \mathrm{~m} / \mathrm{s}, \mathrm{T}_{\mathrm{e}}=0.2 \mathrm{eV}, \phi_{\mathrm{p}}=-20, \mathrm{~m}_{\mathrm{i}}=16 \mathrm{amu}$

Contour values: $v / u_{\infty}= \pm .01, \pm .1, \pm .3, \pm .5$, positive values below, negative values above, from the freestream toward the plate 


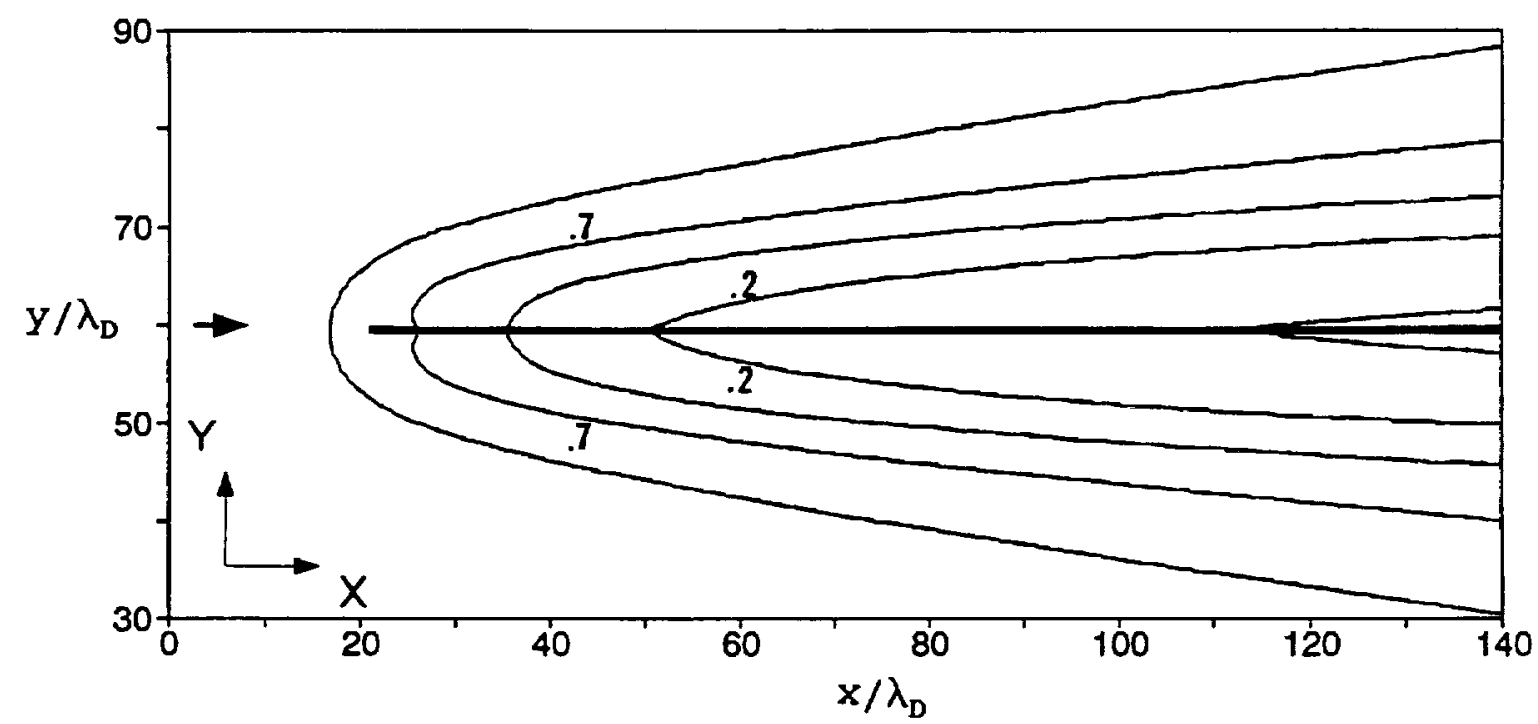

Figure 5. Ionic density contours for a single semi-infinite flat plate: $u_{\infty}=8780 \mathrm{~m} / \mathrm{s}, \mathrm{T}_{\mathrm{c}}=0.2 \mathrm{eV}, \phi_{\mathrm{p}}=-20, \mathrm{~m}_{\mathrm{i}}=16 \mathrm{amu}$ Contour values: $n_{\mathrm{i}} / \mathrm{n}_{\infty}=.99, .7, .368, .2, .1$, from the freestream toward the plate

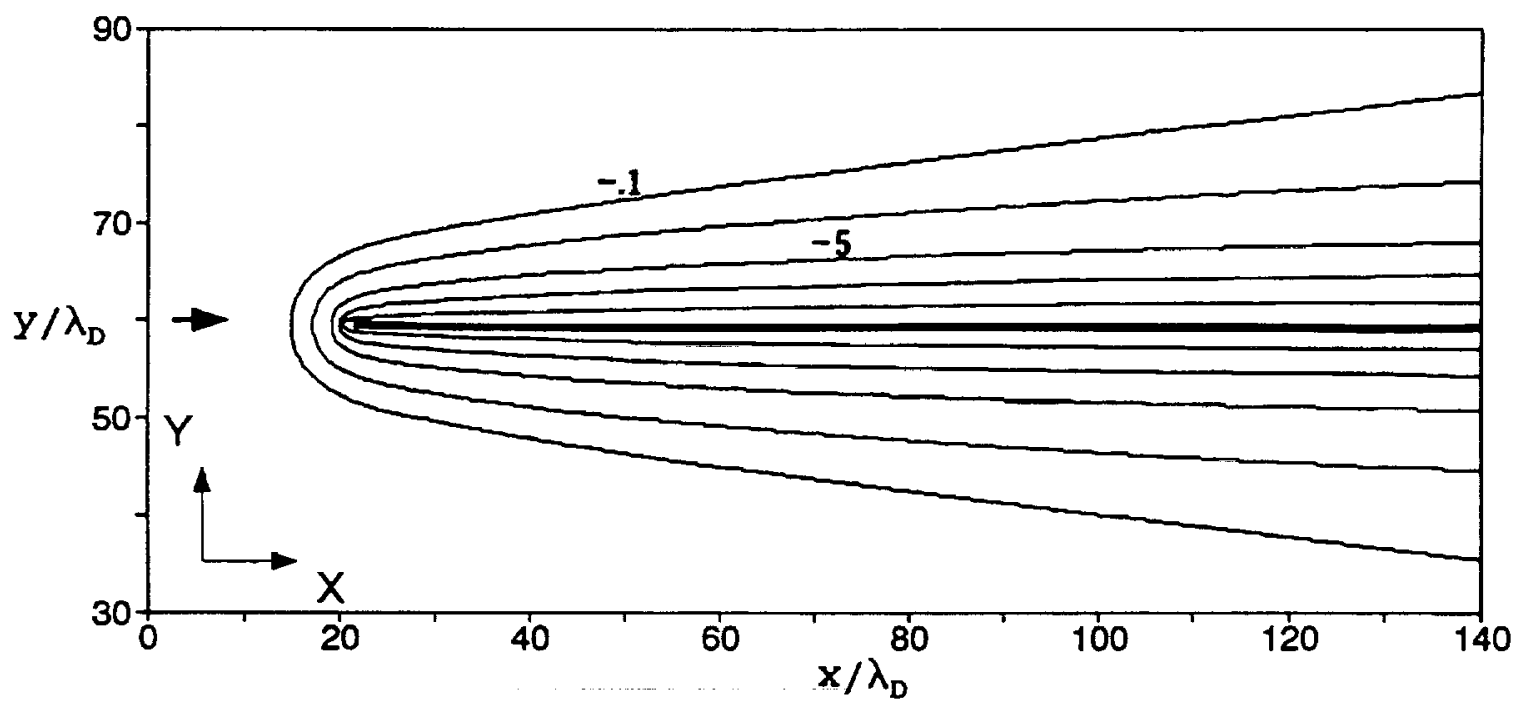

Figure 6. Electrostatic potential contours for a single semiinfinite flat plate: $u_{\infty}=8780 \mathrm{~m} / \mathrm{s}, T_{e}=0.2 \mathrm{eV}, \phi_{p}=-20$, $m_{i}=16$ amu

Contour values: $\phi=-.1,-1,-5,-10,-15$, from the freestream toward the plate 


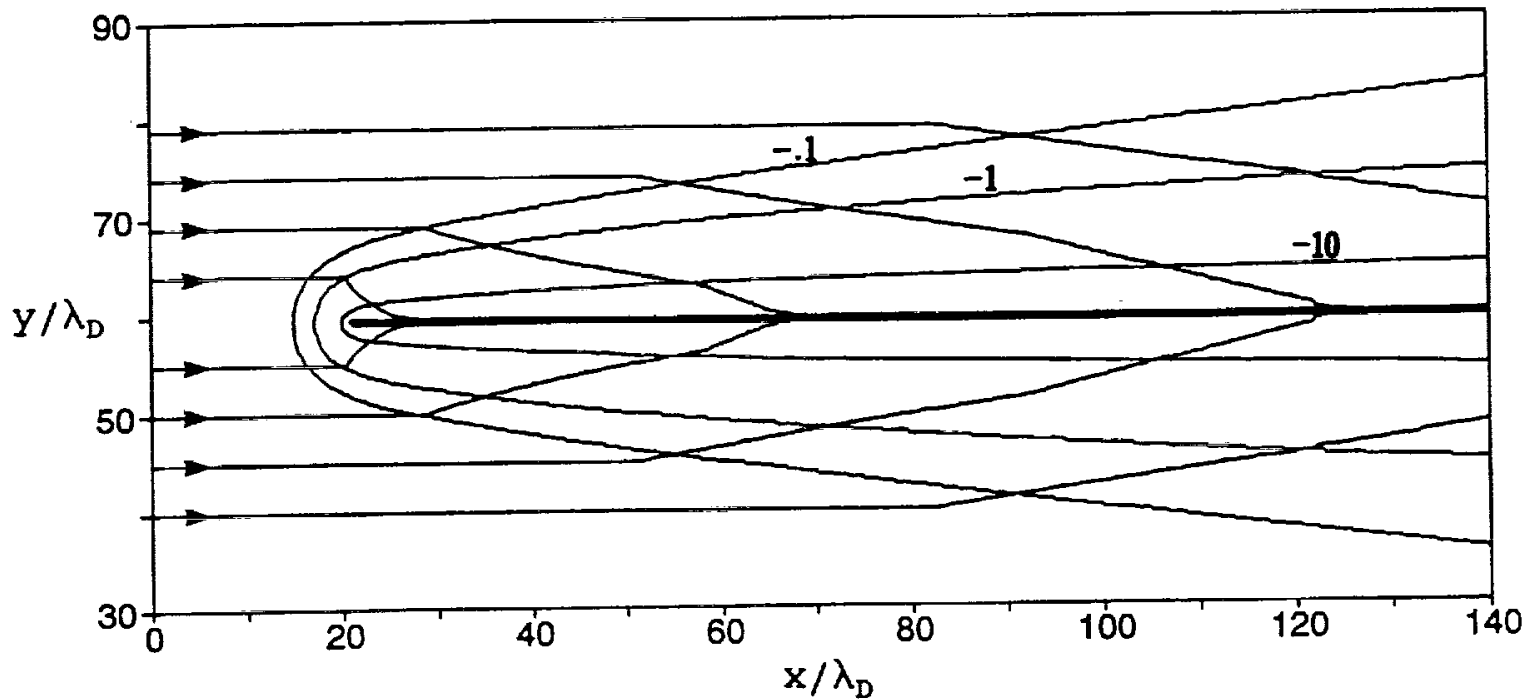

Figure 7. Streamlines superimposed on electric potential contours for a single semi-infinite flat plate: $\mathrm{u}_{\infty}=8780 \mathrm{~m} / \mathrm{s}, \mathrm{T}_{\mathrm{e}}=0.2 \mathrm{eV}, \phi_{\mathrm{p}}=-20, \mathrm{~m}_{\mathrm{i}}=16 \mathrm{amu}$

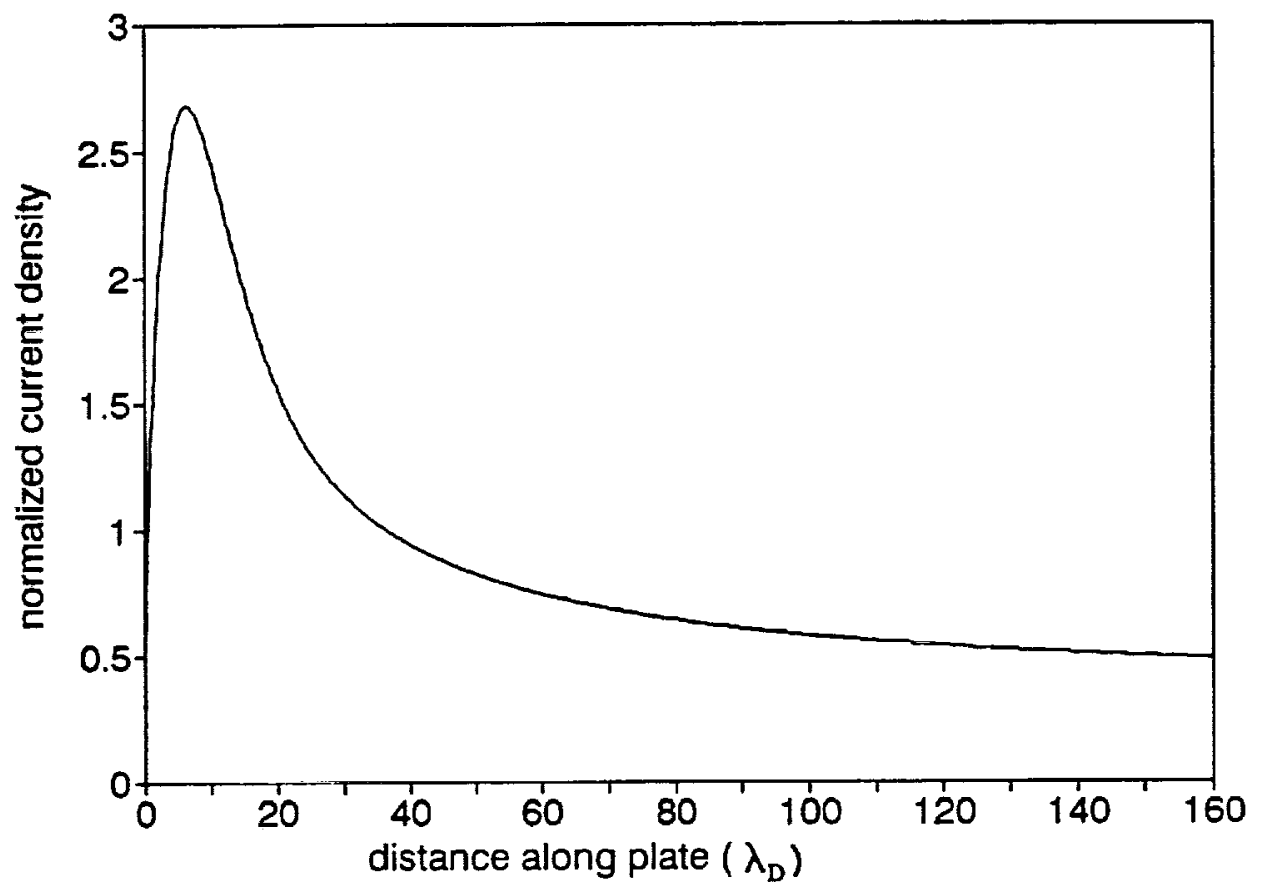

Figure 8. Normalized current density $J /\left(e n_{\infty} u_{\beta}\right)$ to a single semi-infinite flat plate: $u_{\infty}=8780 \mathrm{~m} / \mathrm{s}, T_{e}=0.2 \mathrm{eV}$, $\phi_{p}=-20, m_{i}=16$ amu 
distortion is caused by the streaming source of relatively high ion density of large mass available near the edge. From Poisson's equation, an increase in density compared to the non-flowing case causes a decrease in the sheath dimension. The plate, however, acts as a sink absorbing all perpendicularly incident ions. As the perpendicular velocity increases downstream due to acceleration by the electric field, there is a drop in density as it is absorbed by the plate, and the sheath expands accordingly. Expansion continues further downstream to such an extent that the current passing into the sheath from the freestream is equal to that which is absorbed by the plate. When this point is reached, there are no further changes in velocity or potential in the flow direction, and the flow becomes essentially onedimensional. This region is labeled as 'fully developed.'

Certain flow patterns shown by the figures are worth noting. Figure 3 shows that as one moves downstream from the leading edge, the velocity in the flow direction near the plate decreases which would imply a decelerating force. This, however, is not the case. As one moves downstream in the leading edge region, the vertical velocity is increasing, and convects the slower free-stream parallel flow toward the plate. The result is an overall decrease in the parallel flow velocity. In the region where the vertical convection is taking place, the current density to the plate, which is the product of the density and the vertical velocity, reaches a maximum and then decreases toward a constant value in the 
fully-developed region. Figure 8 shows that the maximum current density occurs approximately 6.3 Debye lengths in from the leading edge for the flow parameters chosen.

From Figures 3 and 4 , it is evident that the ions are accelerated prior to the leading edge by the electrostatic field even though the flow is hypersonic. It should be noted that electromagnetic disturbances travel at the speed of light, and their influence is not limited by speed of sound considerations. Poisson's equation reflects this by being elliptic in nature.

The above results are compared with theoretical and published computational models below.

\section{Analytical Comparison}

For the LEO parameters used above, the Debye length is typically much smaller than spacecraft dimensions. The sheath is then concentrated in a thin layer surrounding the spacecraft. In this situation, the space charge becomes important in determining the characteristics of the problem, and the one-dimensional current flow between the sheath edge and a spacecraft surface is given by the space-charge-limited Child-Langmuir law [ref. 5]

$$
j_{i}=\frac{4 \epsilon_{0}}{9}\left(\frac{2 q}{m_{i}}\right)^{\frac{1}{2}} \frac{\phi^{\frac{3}{2}}}{s^{2}}
$$

where $s$ is the distance from the surface to the edge of the sheath. 
The approximation of a definite sheath edge imposes restrictions on the solution of the governing equations. Mathematically, the restriction states that $v_{i}^{s} \geq\left(k T_{e} / m_{i}\right)^{1 / 2}$ which is known as the Bohm criteria. Physically it requires that in order for a sheath to form around a negatively biased surface, the ions must stream into the sheath boundary with a velocity equal to or greater than $\left(k \mathrm{~T}_{\mathrm{c}} / \mathrm{m}_{\mathrm{i}}\right)^{1 / 2}$. The sheath dimension $\mathrm{s}$ in units of Debye length is given by

$$
s=\frac{1}{0.6} \frac{2^{\frac{5}{4}}}{3}\left(\frac{q \phi}{k T_{e}}\right)^{\frac{3}{4}}\left(\frac{u_{\beta}}{v_{i}^{s}}\right)^{\frac{1}{2}}
$$

The factor of $1 / 0.6$ occurs from the conversion of the Debye length in terms of sheath parameters, to the Debye length in terms of free stream parameters (i.e. $n_{i}^{3}=n_{\infty} e^{-1}$ ). If it is assumed that the Bohm criteria is just satisfied (i.e. $\mathrm{u}_{\beta} / \mathrm{v}_{\mathrm{i}}^{3}=1$ ), then the ion current density to the plate in the fully developed region is found to be independent of the plate potential [ref. 25]

$$
j_{2}=0.37 e n_{\infty} u_{\beta}
$$

These analytical equations can be obtained from both kinetic theory and a hydrodynamic formulation. Wang and Hastings [ref. 25] provide a detailed derivation using the hydrodynamic formulation, and applied the results to the case of a finite-flat plate parallel to a hypersonic plasma flow. The analytical equations are applicable only in the fully developed steady-state sheath far downstream from the leading 
edge of the plate. Wang and Hastings quantified a leading edge ratio assuming $u_{\beta} / v_{i}^{s}=1$ as

$$
\xi=\frac{1.3 M_{0} i \phi_{p} i^{3 / 4}}{\frac{L_{t o t}}{\lambda_{D}}}
$$

and stated that the analytical treatment is sufficient only for plates with $\xi<<1$.

The flow field presented in Figures $3-8$ satisfies the leading edge ratio given by Equation (4.4). Table 1 shows the comparison between analytically predicted values of current density and sheath parameters to those calculated at a point above the plate in the flow field where the Bohm criteria is just satisfied. To test the current density's independence of surface potential, the same problem was run for a surface potential of $\phi_{\mathrm{p}}=-10$ as well. These results are given in Table 2 .

The above analytical approach assumes that the ions are cold. Other theories have been proposed which make different approximations for the ions. A description of a couple of theories and the results they give will help bound the effects introduced in assuming a cold ion distribution [ref. 28].

If the ions are assumed to be in a state of thermodynamic equilibrium, then a distribution function for the ions can be defined much like the Boltzmann factor for the electrons. In order for the ions to be in thermodynamic equilibrium at all points in space for an attracting potential, the surface must be reflecting. As a result, the ion density adjacent to the 
Table I. Comparison between analytical and computed electric sheath parameters for a single semiinfinite flat plate: $u_{\infty}=8780 \mathrm{~m} / \mathrm{s}, T_{e}=0.2 \mathrm{eV}$, $\phi_{p}=-20, m_{i}=16$ amu

\begin{tabular}{|l|c|c|}
\hline & Analytical & Computed \\
\hline Sheath Dimension & $12.3 \lambda_{\mathrm{D}}$ & $14.3 \lambda_{\mathrm{D}}$ \\
\hline Sheath Edge Potential & $-.2 \mathrm{~V}$ & $-.22 \mathrm{~V}$ \\
\hline Sheath Edge Density & $.367 \mathrm{n}_{\mathrm{o}}$ & $.42 \mathrm{n}_{\mathrm{o}}$ \\
\hline $\begin{array}{l}\text { Normalized Plate } \\
\text { Current Density }\end{array}$ & .37 & .48 \\
\hline
\end{tabular}

Table II. Comparison between analytical and computed electric sheath parameters for a single semiinfinite flat plate: $u_{\infty}=8780 \mathrm{~m} / \mathrm{s}, T_{e}=0.2 \mathrm{eV}$, $\phi_{p}=-10, m_{i}=16$ amu

\begin{tabular}{||l|c|c|}
\hline & Analytical & Computed \\
\hline Sheath Dimension & $7.3 \lambda_{D}$ & $10.3 \lambda_{D}$ \\
\hline Sheath Edge Potential & $-.2 \mathrm{~V}$ & $-.21 \mathrm{~V}$ \\
\hline Sheath Edge Density & $.367 \mathrm{n}_{\mathrm{o}}$ & $.41 \mathrm{n}_{\mathrm{o}}$ \\
\hline $\begin{array}{l}\text { Normalized Plate } \\
\text { Current Density }\end{array}$ & .37 & .48 \\
\hline
\end{tabular}


surface is much greater than the free-stream value, and the potential drops off rapidly away from the surface.

A perfectly reflecting wall is not very realistic especially when the surface is metallic. Another approach is to assume that the ion distribution is unaffected by the potential and takes on the free-stream value at all points. In this case, the potential drops off less rapidly than for a reflecting wall.

For an absorbing surface, the adjacent density is less than the free-stream value, and the sheath expands farther than in the previous two cases. If the ions also have a finite temperature, electrical currents can flow even without the presence of an accelerating electric field. The result is an increase of current to a surface and a smaller sheath dimension than in the case of cold ions. The overall effect, however, is small [ref. 25]. By assuming cold ions, the effects of the imposed electric potentials will be only slightly overestimated in the region near the plate.

\section{Floating Potential}

The floating potential of a spacecraft is defined as that electric potential required to balance the ion and electron currents to the spacecraft, that is no net current. This is of course in the absence of any imposed potentials on the spacecraft surfaces due to its operation (i.e. solar power arrays). An estimate of the floating potential for quiescent conditions (i.e. no imposed plasma flow) can be obtained by 
setting relations for the ion and electron current equal to each other [ref. 5].

$$
\begin{gathered}
j_{i}=\frac{1}{2} n_{\infty}\left(\frac{k T_{e}}{m_{i}}\right)^{\frac{1}{2}} \\
j_{e}=\frac{1}{2} n_{\infty}\left(\frac{2 k T_{e}}{\pi m_{e}}\right)^{\frac{1}{2}} e^{\frac{\theta \phi_{f}}{k T_{\theta}}}
\end{gathered}
$$

The electron flux relation is the random electron thermal current times the Boltzmann factor, and the ion flux relation is for monoenergetic ions. For oxygen ions, the above relation specifies $\phi_{\mathrm{f}}$ to be about 4.9 times the thermal energy of the electrons negative relative to space for quiescent conditions. For the present study, the ion current to the plate is obtained from the solution of the governing equations.

$$
j_{i}=\sum_{p} n_{i} v_{i}
$$

where the summation is the flux over the plate. Equating equation (4.6) for the electron flux and equation (4.7), the only unknown is the floating potential which is solved for.

The floating potential was determined for a single finite flat plate parallel to a flowing plasma characteristic of LEO at different altitudes [ref. 2]. The electric potential on the plate was iterated until the resulting ion current matched the thermal electron current at that potential. The results are given in Table 3. 
Table III. Floating potential calculation as a function of orbital altitude for a single finite flat plate

\begin{tabular}{|c|c|c|c|c|c|}
\hline $\begin{array}{c}\text { Altitude } \\
{[\mathrm{km}]}\end{array}$ & $\begin{array}{c}\text { Space- } \\
\text { craft } \\
\text { Velocity } \\
{[\mathrm{m} / \mathrm{s}]}\end{array}$ & $\begin{array}{c}\text { Ion } \\
\text { Mass } \\
{[\text { amu }]}\end{array}$ & $\begin{array}{c}\text { Electron } \\
\text { Temp. } \\
{[\mathrm{eV}]}\end{array}$ & $\begin{array}{c}\text { Calculated } \\
\text { Floating } \\
\text { Potential } \\
{[\mathrm{V}]}\end{array}$ & $\begin{array}{c}\text { \# Times } \\
\text { Electron } \\
\text { Temp }\end{array}$ \\
\hline 200 & 7800 & 18 & .172 & -.74 & 4.3 \\
\hline 300 & 7700 & 16 & .1895 & -.8 & 4.2 \\
\hline 400 & 7700 & 15 & .207 & -.87 & 4.2 \\
\hline 500 & 7600 & 15 & .224 & -.95 & 4.2 \\
\hline 600 & 7600 & 15 & .233 & -.99 & 4.2 \\
\hline 700 & 7500 & 14 & .25 & -1.05 & 4.2 \\
\hline 800 & 7500 & 13 & .258 & -1.09 & 4.2 \\
\hline 900 & 7400 & 10 & .267 & -1.1 & 4.1 \\
\hline 1000 & 7400 & 7 & .276 & -1.11 & 4.0 \\
\hline
\end{tabular}


Computational Comparison

The flow field described in figures 3 through 8 was compared with results given by Wang and Hastings [ref. 25]. Identical approximations are made in the Wang, Hastings paper, but a particle-in-cell model (PIC) which utilizes a microscopic rather than a continuum description was used. The comparison resulted in nearly identical potential field lines. Differences did occur in the region within a few Debye lengths of the leading edge. The present study predicts a maximum current density 6.3 Debye lengths from the leading edge while the Wang and Hastings paper predicted a maximum at the very tip. It is expected that the maximum current will be at some distance down from the leading edge. The flow field in this region was examined earlier in the chapter, and the reason stems from an increasing cross-flow velocity as one moves down the plate.

\section{Two Parallel plates}

\section{Minimum Separation Distance}

Two studies were conducted involving the electrical interaction of two parallel plates through a flowing plasma. The first describes the interaction of two equally biased plates. The minimum separation distance at which the current collected by each plate is unaffected by the presence of the other is determined as a function of the applied potential to 
the plates. This information can be used, for example, by space shuttle experimenters who need to know if the plasma flow field around their experiment will be affected by the electric potential of the shuttle.

The interaction of two equally biased parallel plates is a function of the separation between the plates, the potential applied to the plates, the properties of the plasma including the speed of the oncoming plasma flow, and the length of the plates. In the present study, the potential applied to the plates was allowed to vary for a constant plate length of five Debye lengths and plasma properties characteristic of a 400 kilometer altitude (see Table 3).

If the plates are separated by a large distance, the current collected by both sides of either plate will be equal and essentially undisturbed by the presence of the other plate. As the plates are moved closer, the rate at which ions are collected by the inside surfaces of the plates approaches that at which they are supplied to the inner region by the oncoming plasma flow. The density between the plates decreases relative to the large separation case as a result, causing a decrease in the current to the inside surfaces. The closer the plates are together, the greater the decrease in density between them. The separation distance at which the current to the inside surface of either plate drops by $5 \%$ and $20 \%$ relative to the large separation case was determined for normalized voltages ranging from -1 to -100 . For the plasma properties chosen, this corresponds to an electric bias 
ranging from -.2 to -20 volts. Figure 9 plots the minimum separation distance for a five and twenty percent drop in current in units of Debye length as a function of normalized potential.

A more detailed representation of the interaction is presented in figures 10 through 25 which show the development of the flow field as two plates at a -50 normalized potential approach one another. The figures are in groups of four showing $u, v, \rho$ and $\phi$ contours for plate separations of 29, 19, 14 and 9 Debye lengths. The figures are plotted with identical scaling in terms of Debye length to emphasize the effects of separation distance.

It is evident from figures 10 through 13 that for a large separation, the flow field about each plate is undisturbed by the presence of the other. For a small separation (i.e. figures 22 through 25) however, there is a dramatic shift in the flow field in the region between the plates. Comparing figures 12 and 24 , it is seen that the density decreases in the inner region as ions are collected by the plates at a rate faster than they are supplied by the oncoming plasma flow. As a result, there is a decrease in current to the inside surfaces. Figure 26 shows this by comparing the normalized current density along the inside surface of the plates to that of a single plate at a -50 normalized potential immersed in an identical plasma flow. The current to the outside surface of the plates remains nearly unchanged compared to the single plate result for all separation distances. 


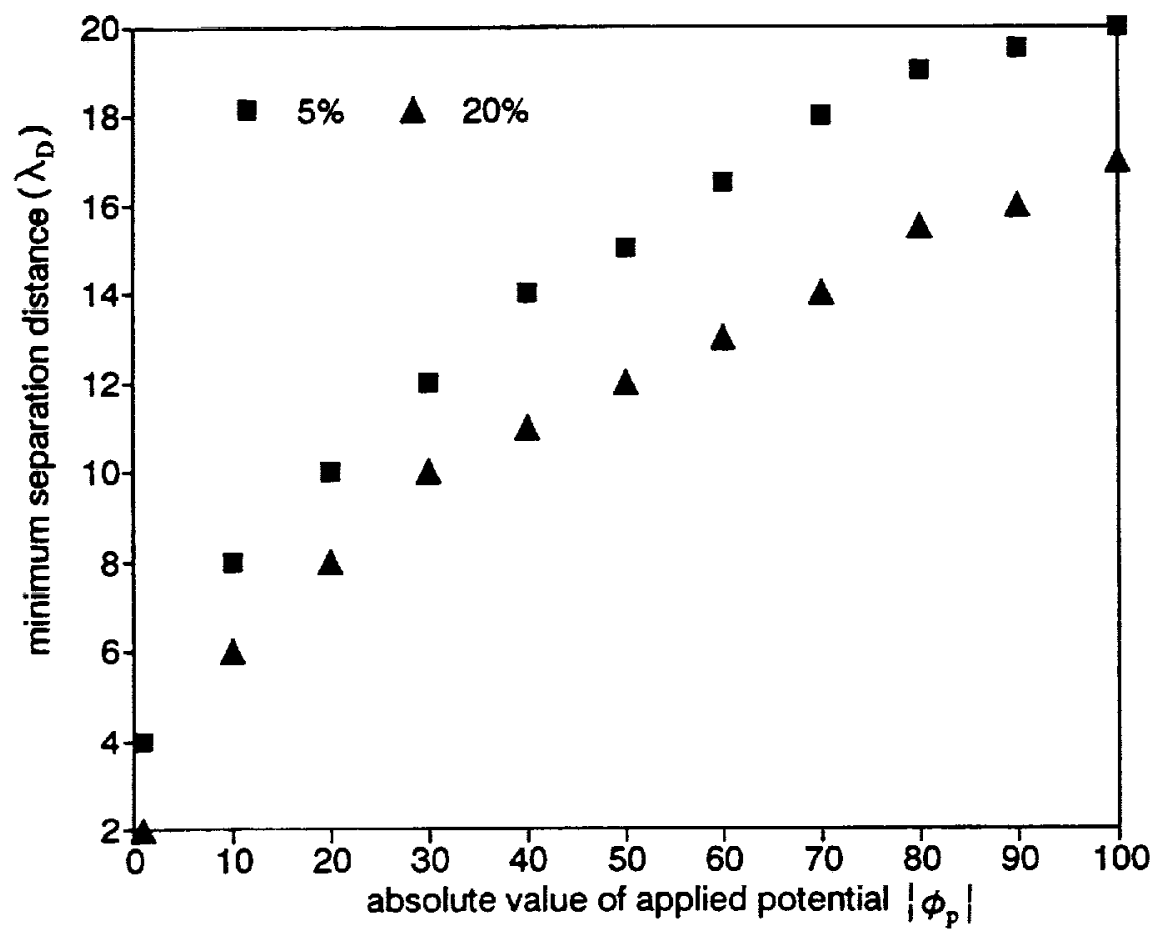

Figure 9. Minimum interaction separation distance between two equally biased flat plates as a function of applied potential: $u_{\infty}=7800 \mathrm{~m} / \mathrm{s}, T_{e}=0.2 \mathrm{eV}, d_{p}=5 \lambda_{D}, m_{i}=16$ amu 
Fig. 10

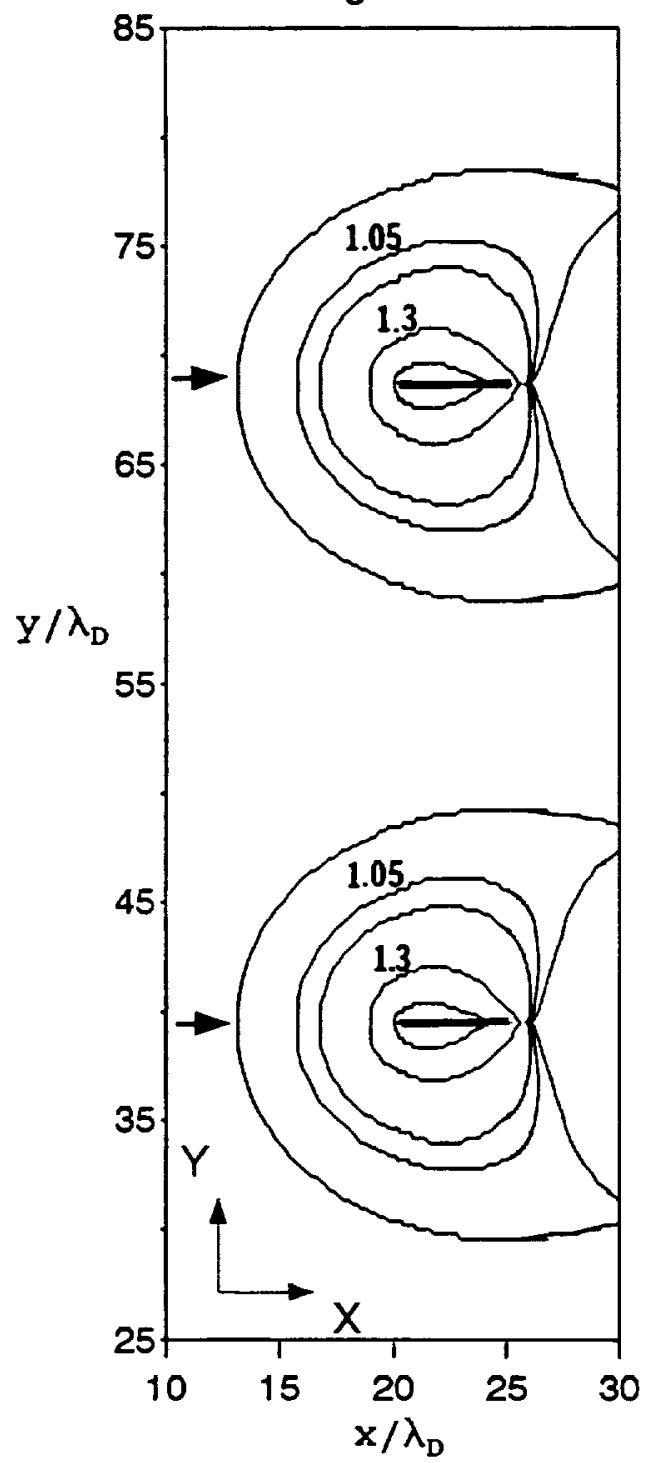

Fig. 11

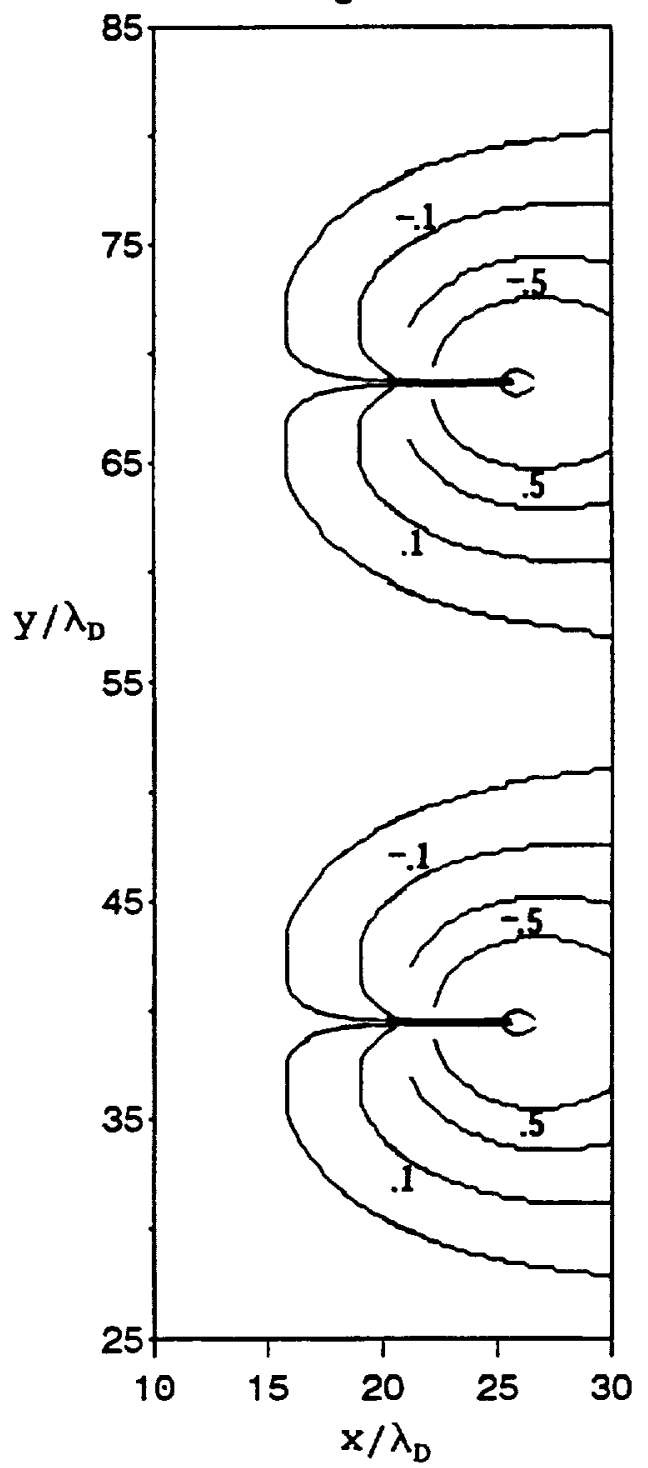

Figure 10. u velocity contours for two equally biased flat plates: $d_{\text {sep }}=29 \lambda_{D}, d_{p}=5 \lambda_{D}, \phi_{p}=-50, u_{\infty}=7800 \mathrm{~m} / \mathrm{s}$, $\mathrm{T}_{\mathrm{e}}=0.2 \mathrm{eV}, \mathrm{m}_{\mathrm{i}}=16 \mathrm{amu}$

Contour values: $u / u_{\infty}=1.005,1.05,1.1,1.3,1.5$ from the freestream toward the plates

Figure 11. v velocity contours for two equally biased flat plates: $d_{s e p}=29 \lambda_{D}, d_{p}=5 \lambda_{D}, \phi_{p}=-50, u_{\infty}=7800 \mathrm{~m} / \mathrm{s}$, $T_{e}=0.2 \mathrm{eV}, \mathrm{m}_{\mathrm{i}}=16 \mathrm{amu}$

Contour values: $v / u_{\infty}= \pm .01, \pm .1, \pm .3, \pm .5, \pm 1$ positive values below the plates, negative values above from the freestream toward the plates 
Fig. 12

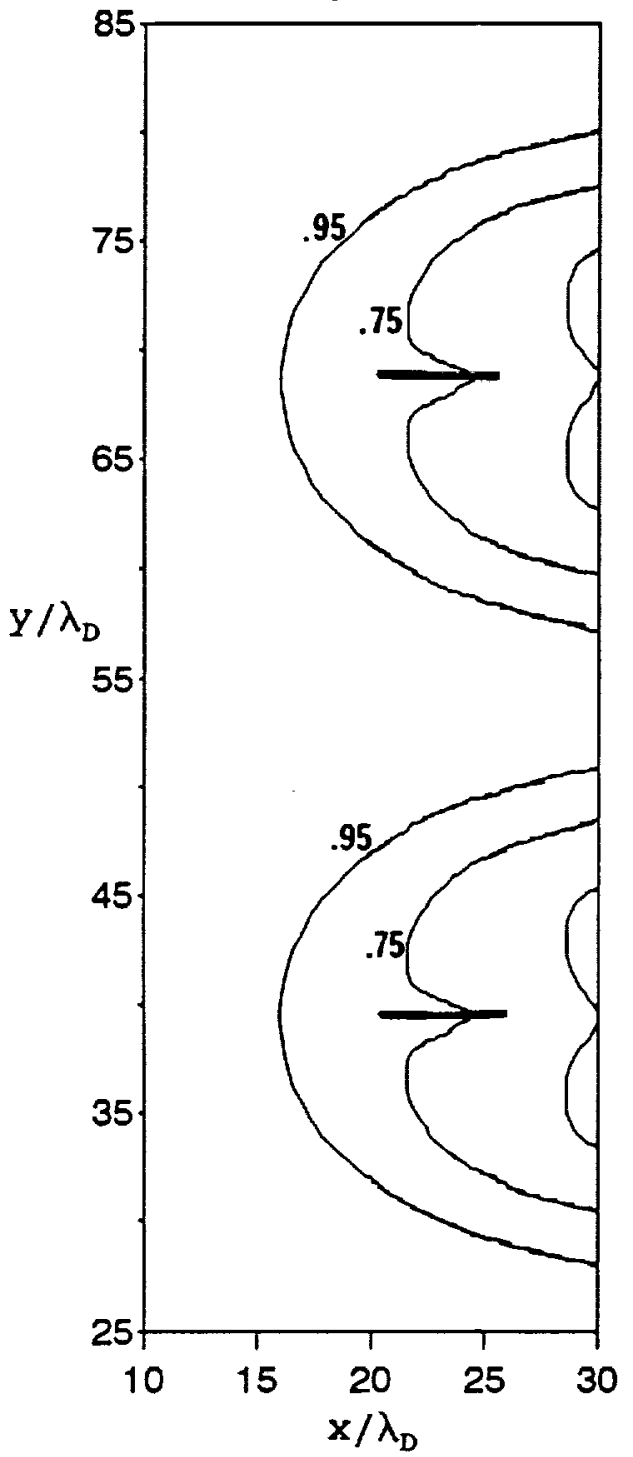

Fig. 13

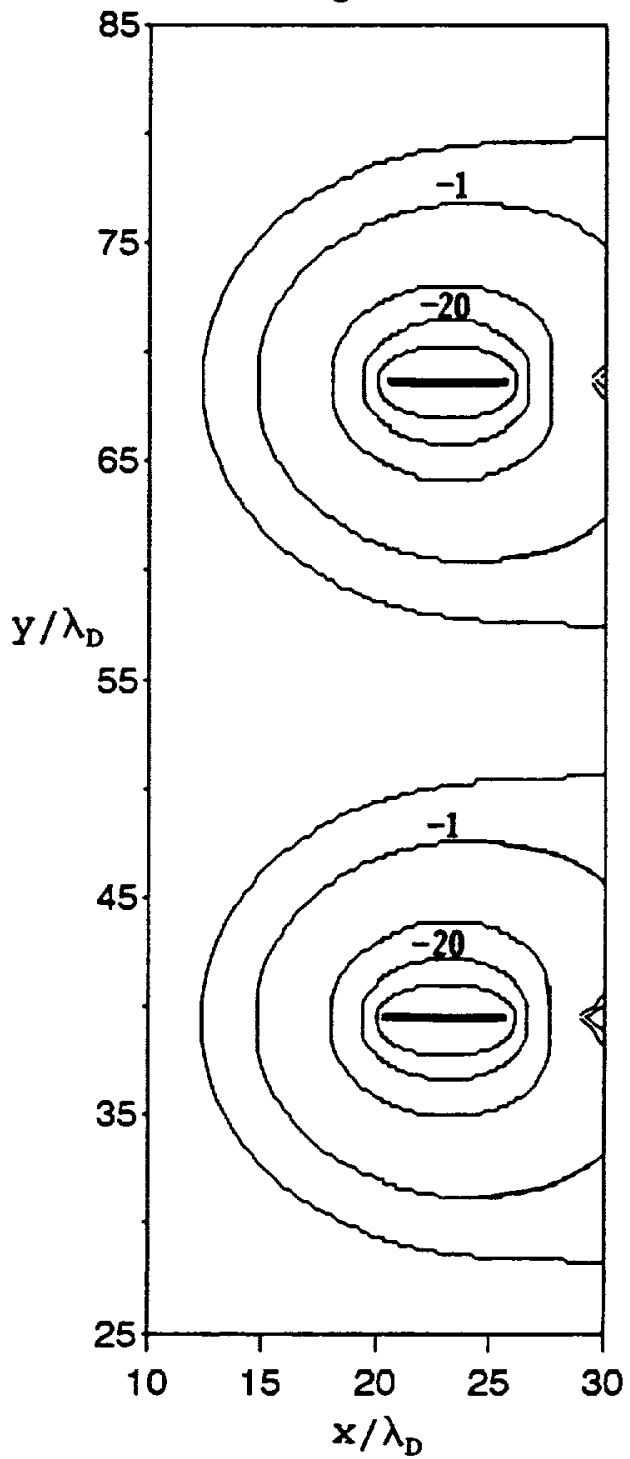

Figure 12. Ionic density contours for two equally biased flat plates: $d_{s e p}=29 \lambda_{D}, d_{p}=5 \lambda_{D}, \phi_{p}=-50, u_{\infty}=7800 \mathrm{~m} / \mathrm{s}$, $\mathrm{T}_{\mathrm{e}}=0.2 \mathrm{eV}, \mathrm{m}_{\mathrm{i}}=16 \mathrm{amu}$

Contour values: $n_{\mathrm{j}} / \mathrm{n}_{\infty}=.95, .75, .5$ from the freestream toward the plates

Figure 13. Electrostatic potential contours for two equally biased flat plates: $d_{2 p p}=29 \lambda_{D}, d_{p}=5 \lambda_{D}, \phi_{p}=-50$, $u_{\infty}=7800 \mathrm{~m} / \mathrm{s}, T_{c}=0.2 \mathrm{eV}, m_{i}=16$ amu

Contour values: $\phi=-.1,-1,-10,-20,-30$ from the freestream toward the plates 
Fig. 14

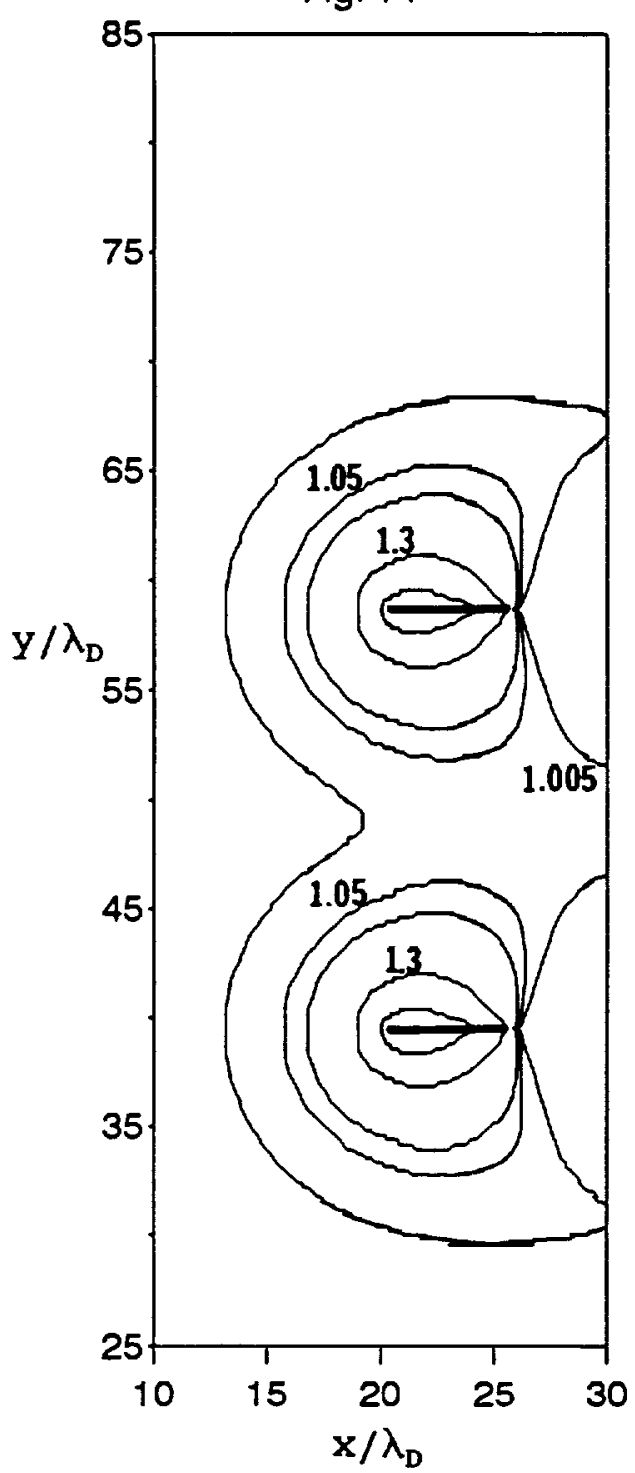

Fig. 15

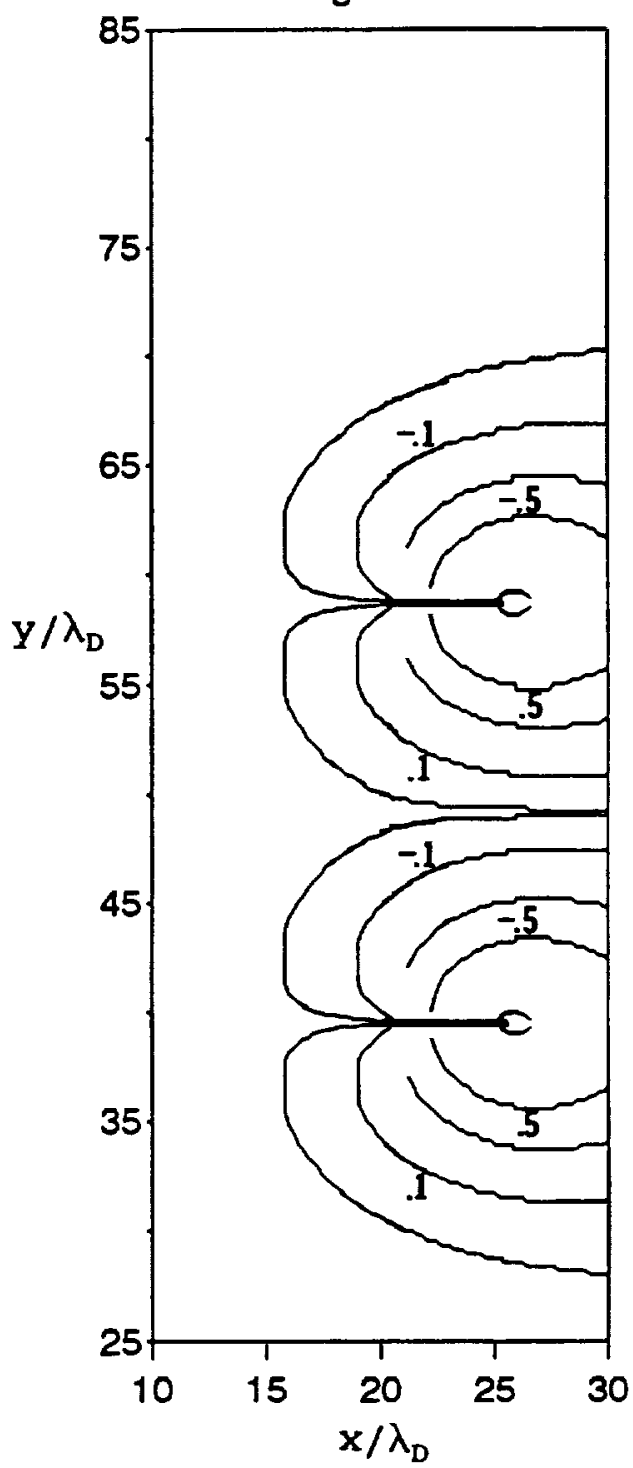

Figure 14. u velocity contours for two equally biased flat plates: $d_{s e p}=19 \lambda_{D}, d_{p}=5 \lambda_{D}, \phi_{p}=-50, u_{\infty}=7800 \mathrm{~m} / \mathrm{s}$, $T_{c}=0.2 \mathrm{eV}, \mathrm{m}_{\mathrm{i}}=16$ amu

Contour values: $u / u_{\infty}=1.005,1.05,1.1,1.3,1.5$ from the freestream toward the plates

Figure 15. v velocity contours for two equally biased flat plates: $d_{s e p}=19 \lambda_{D}, d_{p}=5 \lambda_{D}, \phi_{p}=-50, u_{\infty}=7800 \mathrm{~m} / \mathrm{s}$, $\mathrm{T}_{\mathrm{e}}=0.2 \mathrm{eV}, \mathrm{m}_{\mathrm{i}}=16 \mathrm{amu}$

Contour values: $v / u_{\infty}= \pm .01, \pm .1, \pm .3, \pm .5, \pm 1$ positive values below the plates, negative values above from the freestream toward the plates 
Fig. 16

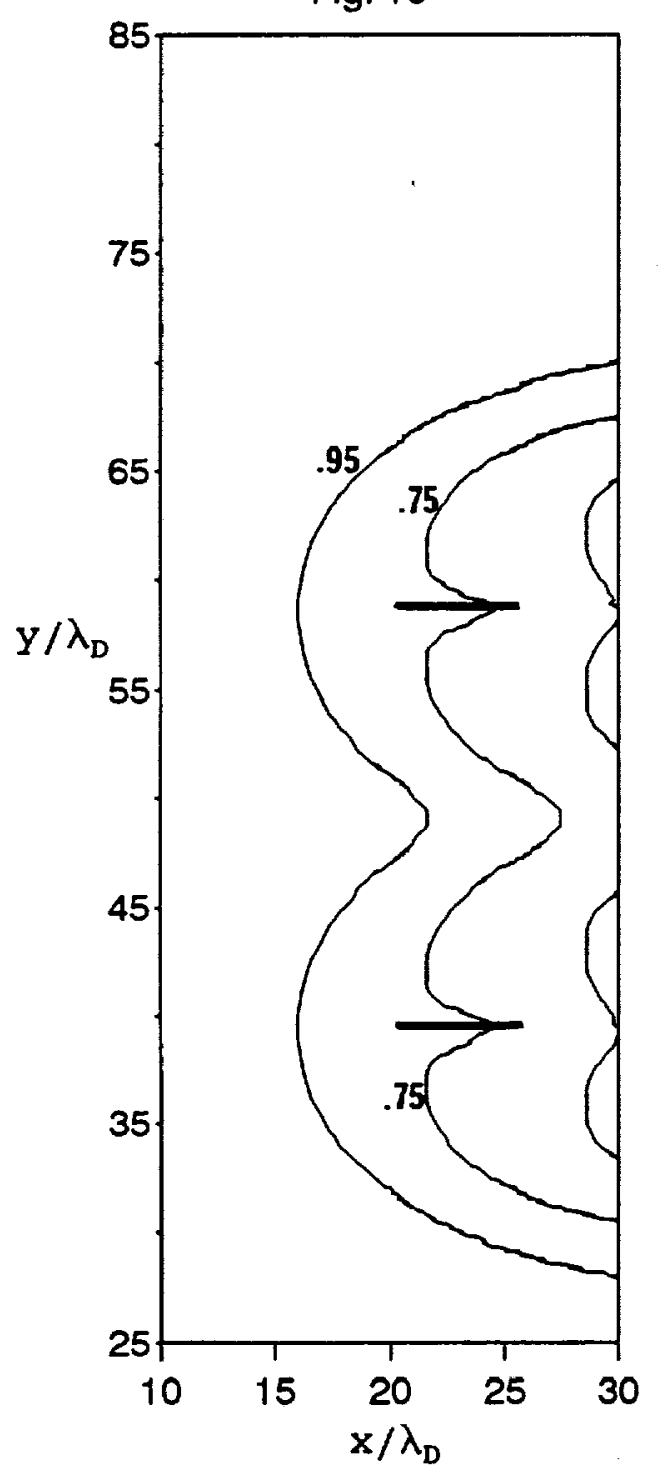

Fig. 17

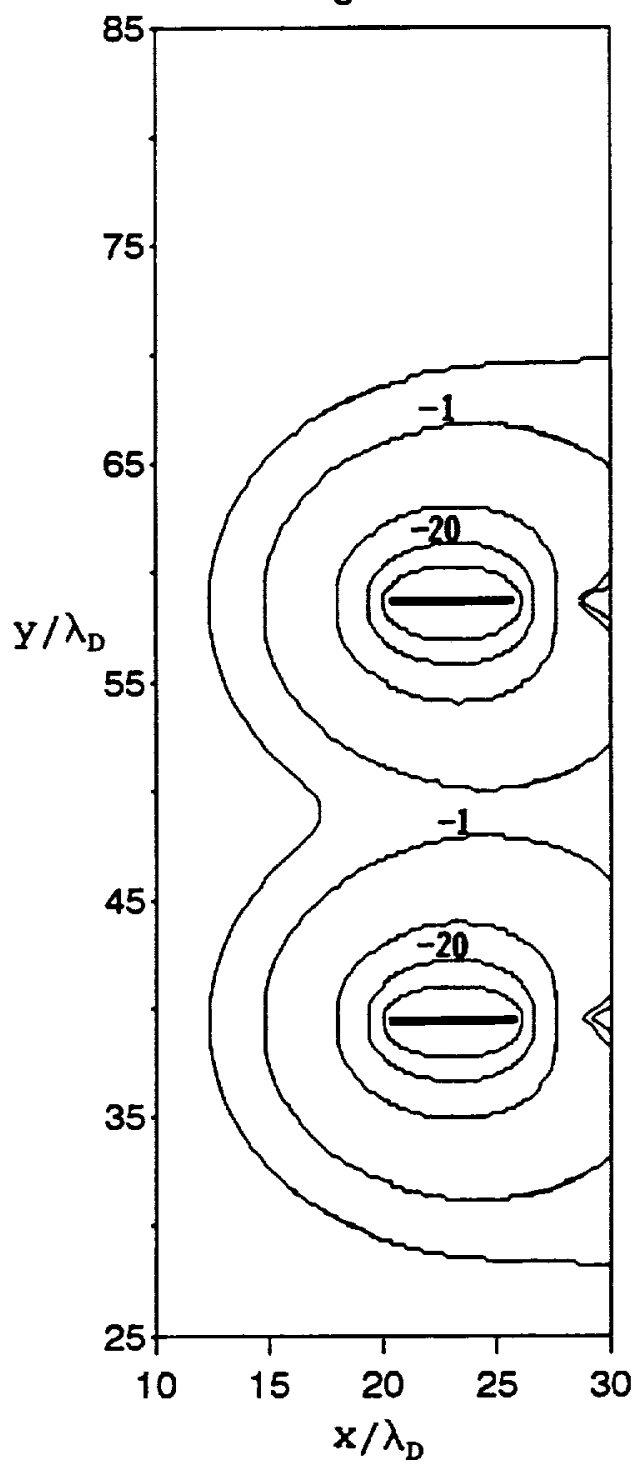

Figure 16. Ionic density contours for two equally biased flat plates: $\alpha_{x c p}=19 \lambda_{D}, d_{p}=5 \lambda_{D}, \phi_{p}=-50, u_{\infty}=7800 \mathrm{~m} / \mathrm{s}$, $\mathrm{T}_{\mathrm{e}}=0.2 \mathrm{eV}, \mathrm{m}_{\mathrm{i}}=16 \mathrm{amu}$

Contour values: $n_{i} / n_{\infty}=.95, .75, .5$ from the freestream toward the plates

Figure 17. Electrostatic potential contours for two equally biased flat plates: $d_{s c p}=19 \lambda_{D}, d_{p}=5 \lambda_{D}, \phi_{p}=-50$, $u_{\infty}=7800 \mathrm{~m} / \mathrm{s}, \mathrm{T}_{\mathrm{e}}=0.2 \mathrm{eV}, \mathrm{m}_{\mathrm{i}}=16 \mathrm{amu}$

Contour values: $\phi=-.1,-1,-10,-20,-30$ from the freestream toward the plates 
Fig. 18

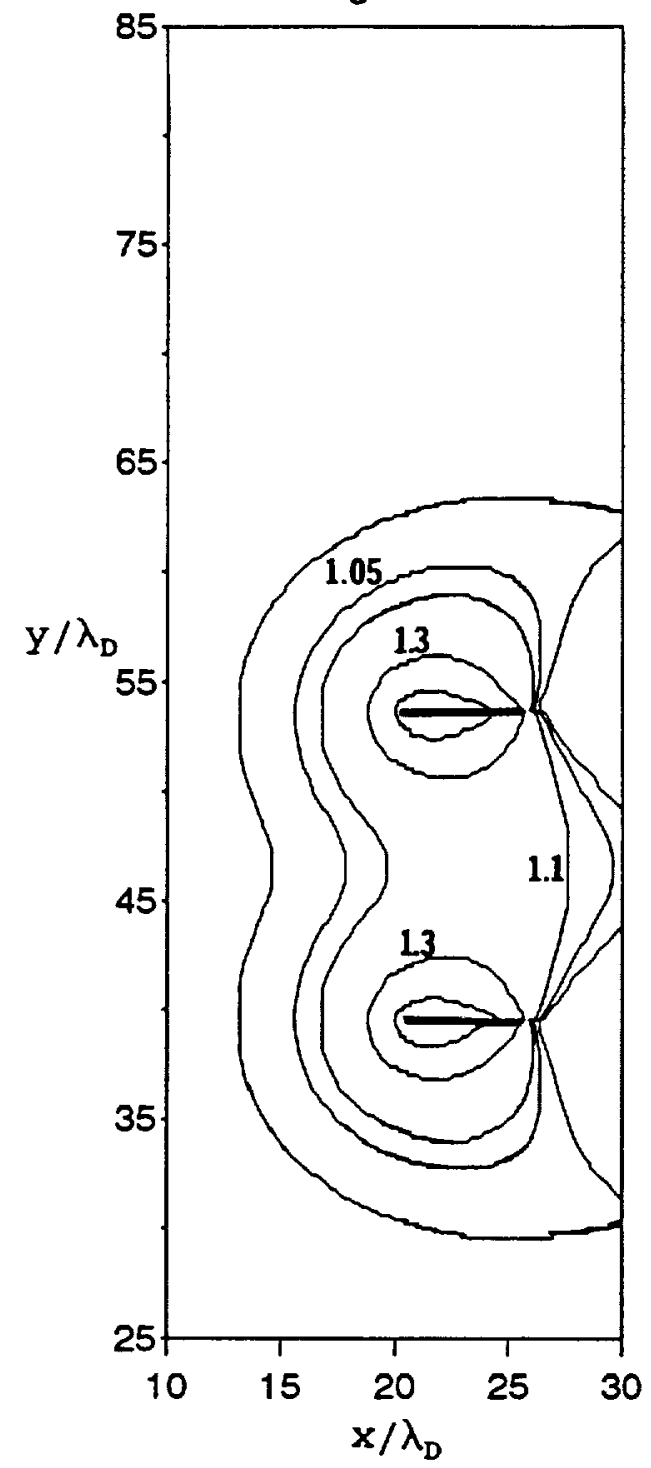

Fig. 19

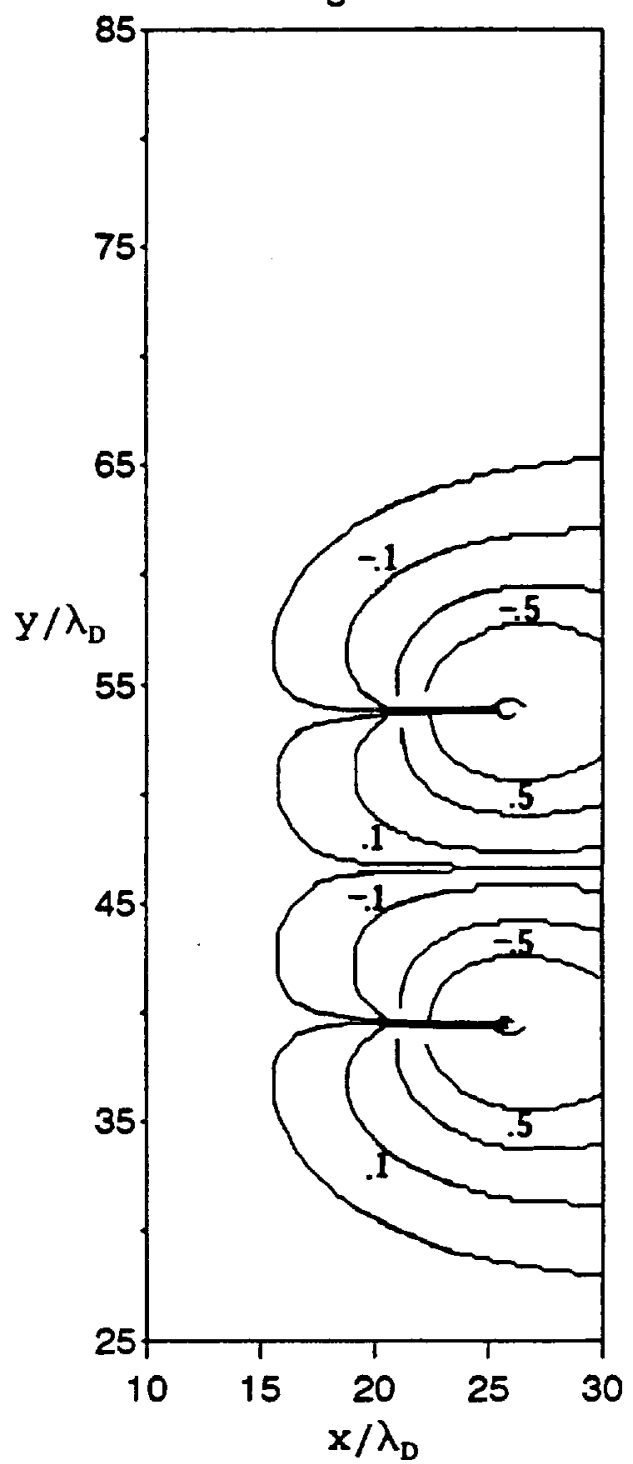

Figure 18. u velocity contours for two equally biased flat plates: $d_{\text {sep }}=14 \lambda_{D}, d_{p}=5 \lambda_{D}, \phi_{p}=-50, u_{\infty}=7800 \mathrm{~m} / \mathrm{s}$, $\mathrm{T}_{\mathrm{c}}=0.2 \mathrm{eV}, \mathrm{m}_{\mathrm{i}}=16 \mathrm{amu}$

Contour values: $u / u_{\infty}=1.005,1.05,1.1,1.3,1.5$ from the freestream toward the plates

Figure 19. v velocity contours for two equally biased flat plates: $d_{\text {sep }}=14 \lambda_{D}, d_{p}=5 \lambda_{D}, \phi_{p}=-50, u_{\infty}=7800 \mathrm{~m} / \mathrm{s}$, $T_{c}=0.2 \mathrm{eV}, \mathrm{m}_{j}=16$ amu

Contour values: $v / u_{\infty}= \pm .01, \pm .1, \pm .3, \pm .5, \pm 1$ positive values below the plates, negative values above from the freestream toward the plates 
Fig. 20

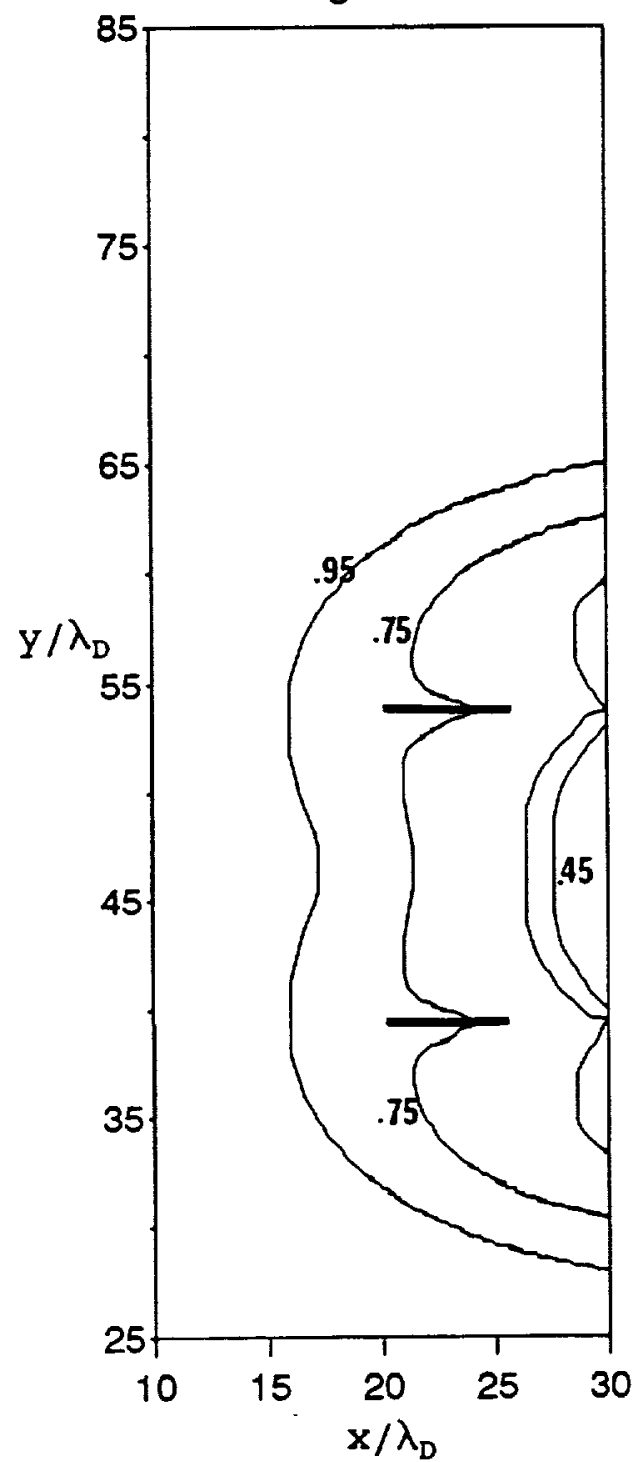

Fig. 21

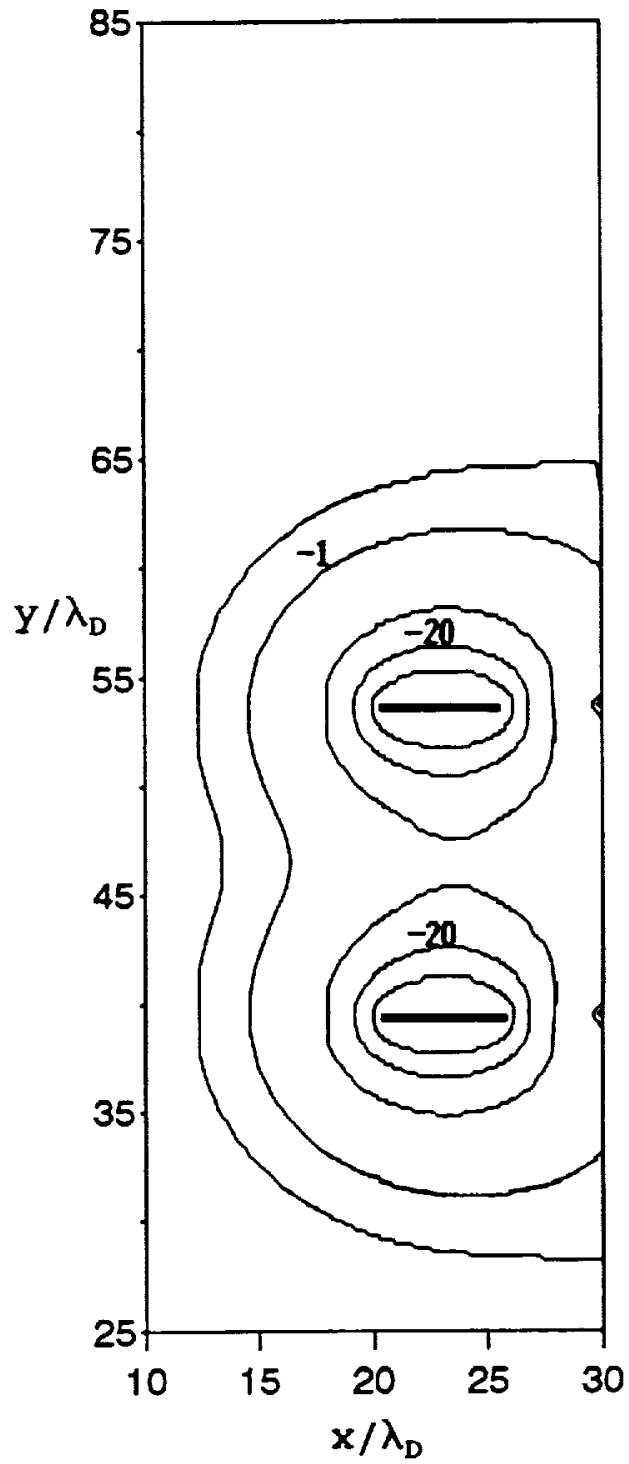

Figure 20. Ionic density contours for two equally biased flat plates: $d_{\text {sep }}=14 \lambda_{D}, \alpha_{p}=5 \lambda_{D}, \phi_{p}=-50, u_{\infty}=7800 \mathrm{~m} / \mathrm{s}$, $\mathrm{T}_{\mathrm{e}}=0.2 \mathrm{eV}, \mathrm{m}_{\mathrm{i}}=16 \mathrm{amu}$

Contour values: $n_{i} / n_{\infty}=.95, .75, .5, .45$ from the freestream toward the plates

Figure 21. Electrostatic potential contours for two equally biased flat plates: $d_{s c p}=14 \lambda_{D}, \alpha_{p}=5 \lambda_{D}, \phi_{p}=-50$, $u_{\infty}=7800 \mathrm{~m} / \mathrm{s}, \mathrm{T}_{\mathrm{c}}=0.2 \mathrm{eV}, \mathrm{m}_{\mathrm{i}}=16 \mathrm{amu}$

Contour values: $\phi=-.1,-1,-10,-20,-30$ from the freestream toward the plates 
Fig. 22

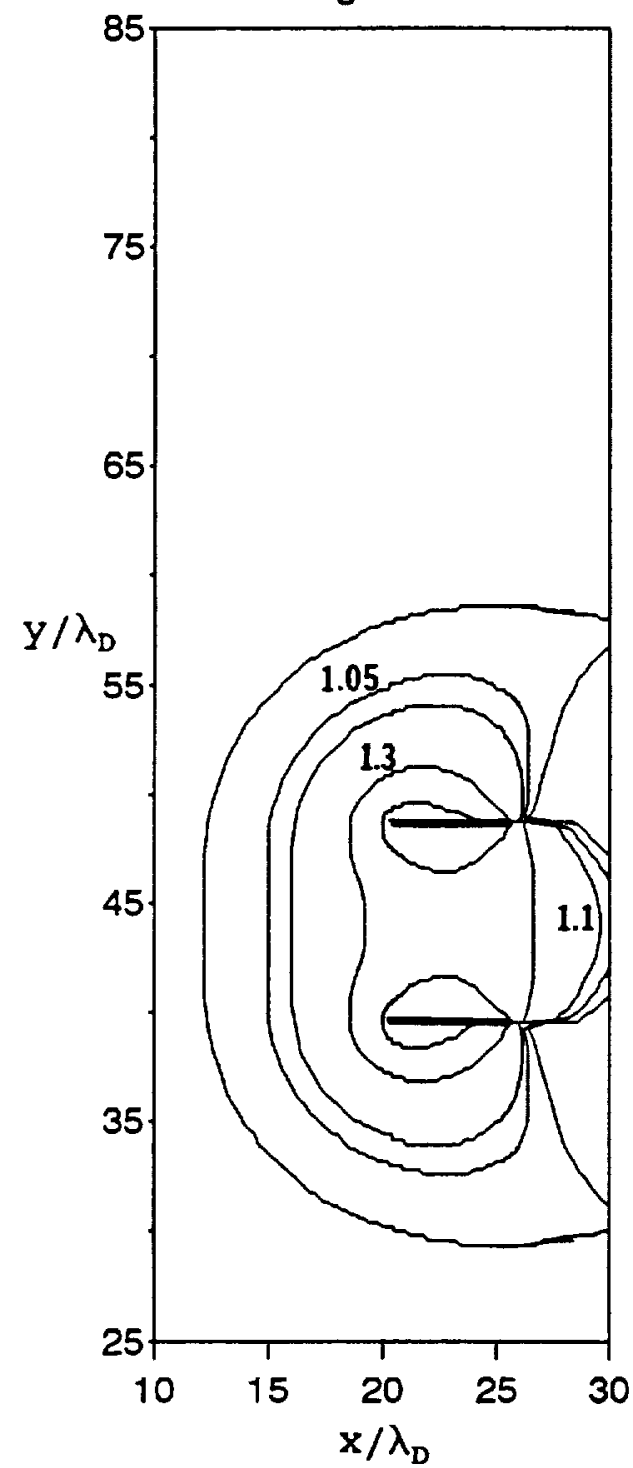

Fig. 23

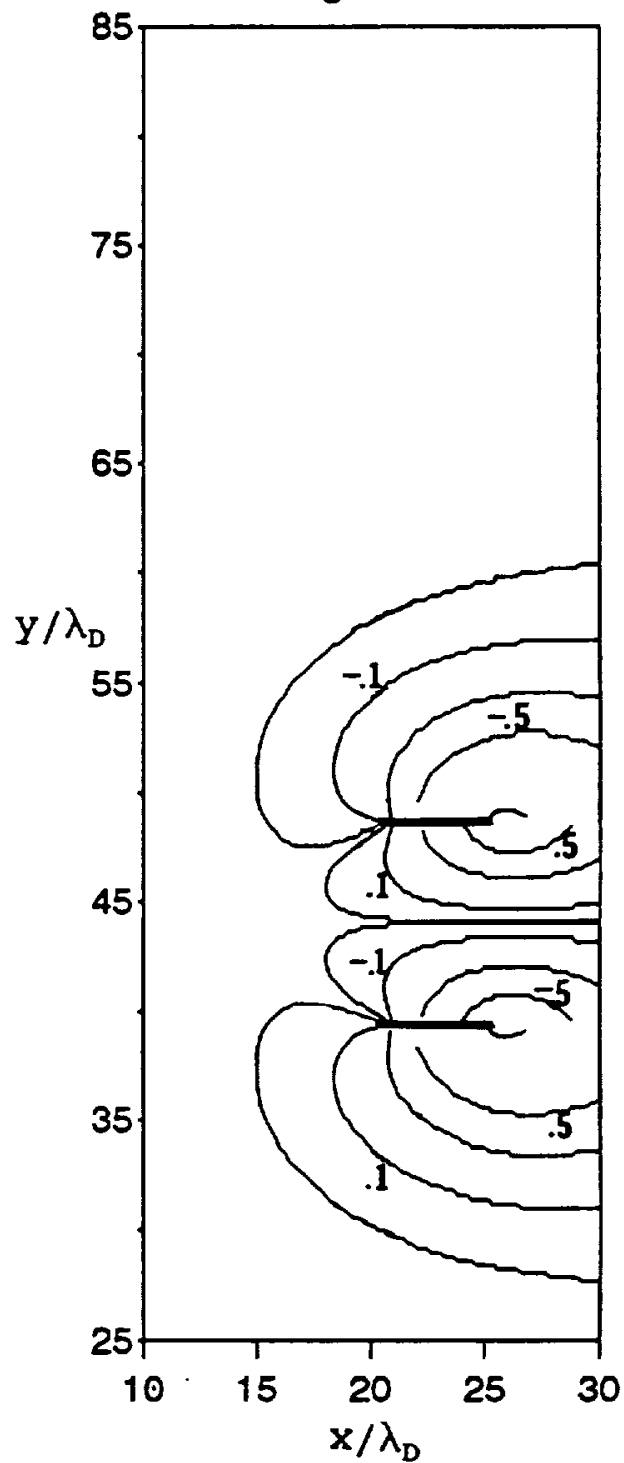

Figure 22. u velocity contours for two equally biased flat plates: $d_{2 c p}=9 \lambda_{D}, d_{p}=5 \lambda_{D}, \phi_{p}=-50, u_{\infty}=7800 \mathrm{~m} / \mathrm{s}$, $\mathrm{T}_{\mathrm{e}}=0.2 \mathrm{eV}, \mathrm{m}_{\mathrm{i}}=16 \mathrm{amu}$

Contour values: $u / u_{\infty}=1.005,1.05,1.1,1.3,1.5$ from the freestream toward the plates

Figure 23. $v$ velocity contours for two equally biased flat plates: $d_{2 c p}=9 \lambda_{D}, d_{p}=5 \lambda_{D}, \phi_{p}=-50, u_{\infty}=7800 \mathrm{~m} / \mathrm{s}$, $\mathrm{T}_{\mathrm{e}}=0.2 \mathrm{eV}, \mathrm{m}_{\mathrm{i}}=16$ amu

Contour values: $v / u_{\infty}= \pm .01, \pm .1, \pm .3, \pm .5, \pm 1$ positive values below the plates, negative values above from the freestream toward the plates 
Fig. 24

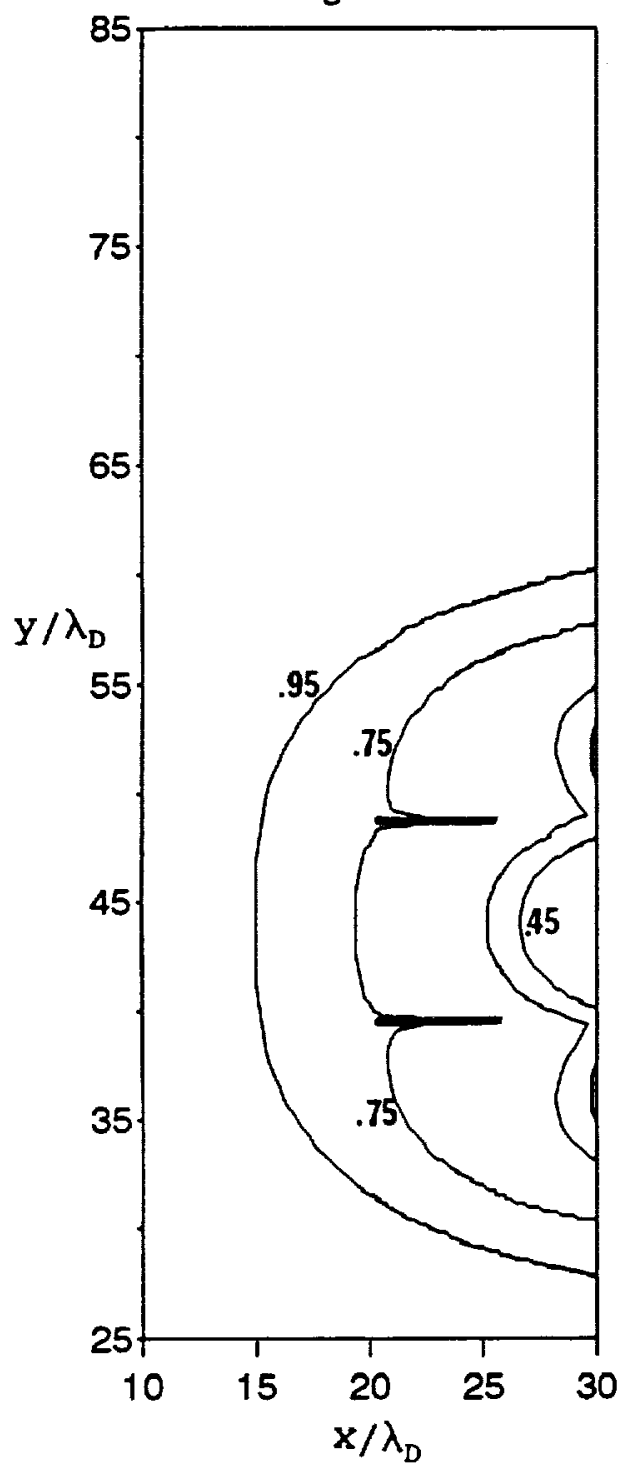

Fig. 25

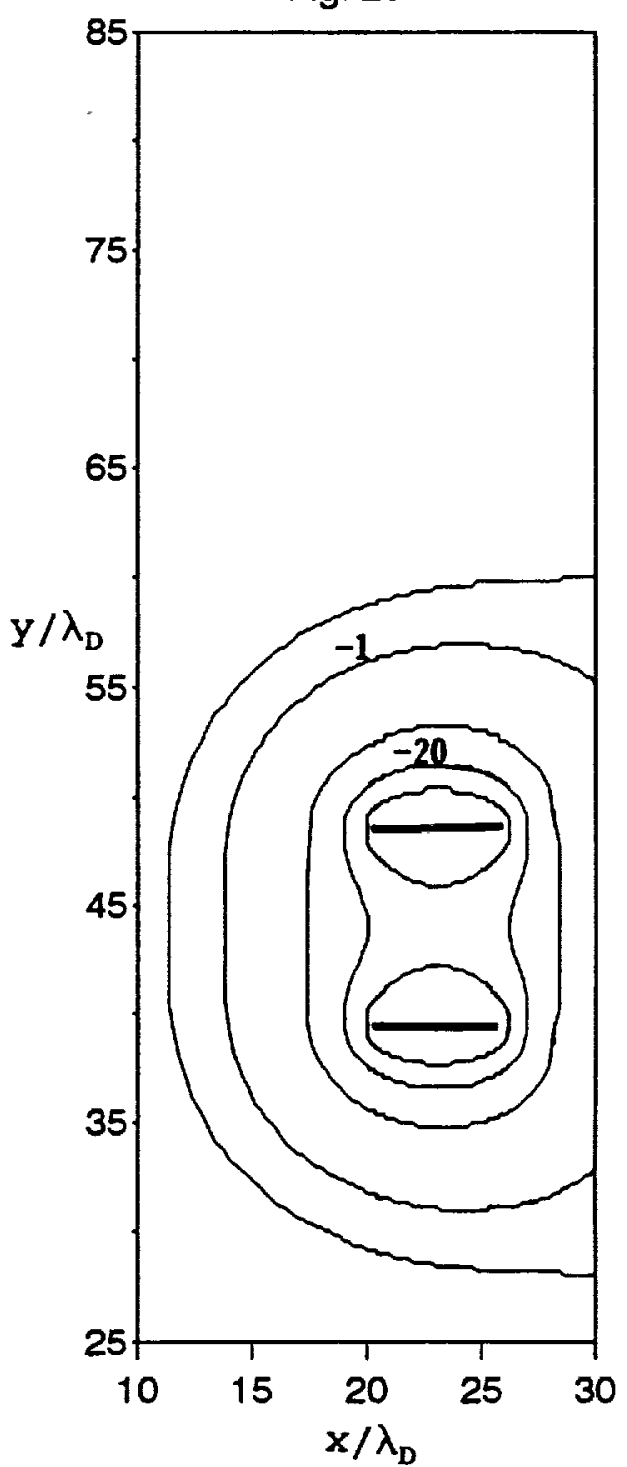

Figure 24. Ionic density contours for two equally biased flat plates: $d_{s c p}=9 \lambda_{D}, d_{p}=5 \lambda_{D}, \phi_{p}=-50, u_{\infty}=7800 \mathrm{~m} / \mathrm{s}$, $\mathrm{T}_{\mathrm{e}}=0.2 \mathrm{eV}, \mathrm{m}_{\mathrm{i}}=16 \mathrm{amu}$

Contour values: $n_{i} / n_{\infty}=.95, .75, .5, .45$ from the freestream toward the plates

Figure 25. Electrostatic potential contours for two equally biased flat plates: $d_{s c p}=9 \lambda_{D}, d_{p}=5 \lambda_{D}, \phi_{p}=-50$, $\mathrm{u}_{\infty}=7800 \mathrm{~m} / \mathrm{s}, \mathrm{T}_{\mathrm{e}}=0.2 \mathrm{eV}, \mathrm{m}_{\mathrm{i}}=16 \mathrm{amu}$

Contour values: $\phi=-.1,-1,-10,-20,-30$ from the freestream toward the plates 


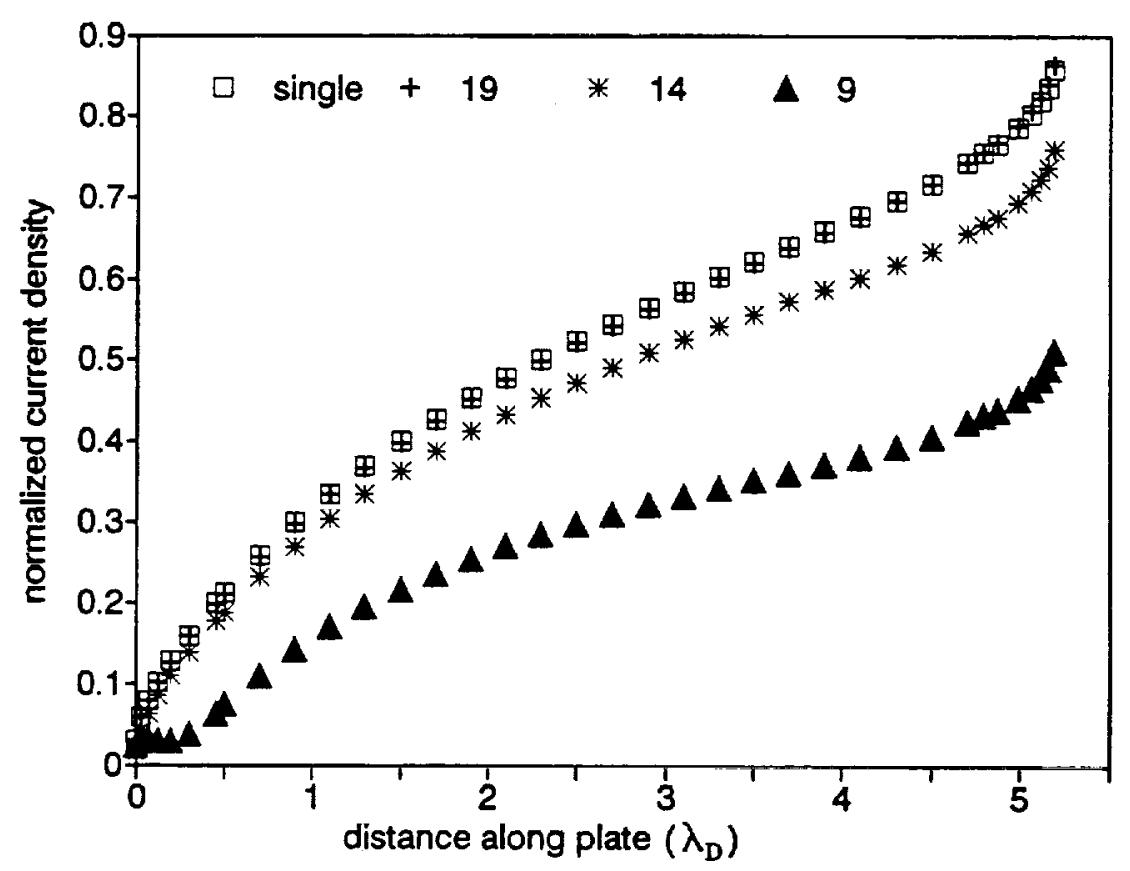

Figure 26. Normalized current density $J /\left(e n_{\infty} u_{\beta}\right)$ to the inside surfaces of two equally biased flat plates for different separation distances: $d_{p}=5 \lambda_{D}, \phi_{p}=-50$, $u_{\infty}=7800 \mathrm{~m} / \mathrm{s}, T_{c}=0.2 \mathrm{eV}, m_{i}=16 \mathrm{amu}$ 
The buildup of density downstream on the axes of the plates due to the focusing of the ions was purposely excluded from the figures showing ionic density contours. Since temperature and pressure were neglected, the only mechanism to dissipate a large density buildup is the electric field force. within the region plotted in the figures, the buildup is not large enough to affect the potential field appreciably; it is not large enough to cause the flow toward the axes to reverse direction. The buildup is very abrupt until enough ions can be accumulated to produce a positive potential and reverse the flow of ions away from the axes. Because the buildup is so abrupt, all the density contour values are present within a few grid points on the axes of the plates but were left out of the figures showing ionic density contours for clarity.

Scaling rules which apply to this problem are determined by considering the form of the governing equations. The problem will depend on the ratios of Debye length to plate length, electric potential to electron temperature and the Mach number. These parameters and their effects on the characteristics of the interaction will be discussed briefly.

The longer the plates, the greater the decrease in the density between them for a constant separation. Therefore, it is expected that the curves in figure 9 would shift toward greater separation distances for increasing plate length.

The electron temperature shows up in three places. First, the dependence of the interaction on the Debye length, which is a function of the electron temperature defined by 
equation (3.4), was addressed in the previous paragraph. It should be noted that the Debye length is also a function of the neutral density which does not show up explicitly in the normalized governing equations. Therefore, the ratio of the Debye length to plate length can be held constant by holding the ratio of the electron temperature to neutral density constant.

In the momentum equations, the Mach number, which is the ratio of the freestream velocity and the ion sound speed, determines the extent of influence of the potential gradient on the ion velocity. Equation (3.3) defines the ion sound speed in terms of the electron temperature. Increasing the Mach number decreases the effect of the potential field causing a spatial compression of the potential field lines. The separation curves in figure 9 would shift toward smaller distances for increasing Mach number.

Finally, the electric potential was normalized by the electron temperature. This procedure was partially responsible for the formation of the Mach number in the momentum equations. The dependence on the electron temperature however, is not fully addressed by the Mach number. In order to have similar flows, the terms on the right hand side of the momentum and Poisson's equations must be the same. This requires that both the ratio of the electric potential to the electron temperature and the Mach number be similar. For example, suppose that two flow problems have the same Mach number but different normalized 
potential. This could be accomplished by changing the electron temperature but holding the Mach number constant by altering either the oncoming flow speed or the ion mass to compensate. Although the Mach number is the same the flows will not be. It is expected that increasing the ratio of electric potential to electron temperature will in general cause the separation curve to shift toward greater distances.

\section{Spacecraft Docking}

The second case study conducted simulates the docking of two spacecraft in orbit. Two parallel plates of unequal potential are moved toward one another in a quasi-static manner. The top plate is held at a relatively large constant negative potential while the other is allowed to 'float' in the resulting potential field. The floating condition is determined by a balance of current to the plate. This problem is applicable to the docking of the space shuttle with the proposed space station Freedom. The space station will have a relatively large constant negative potential due to solar array operation. The shuttle has no external power system which interacts with the space environment and will attain a potential determined mainly by a balance of ambient current to its surfaces. As the two approach however, the potential of the shuttle will adjust so that upon contact the two will form a single system. Results are presented below which describe the adjustment procedure of the floating plate for a plate length of five Debye lengths and plasma properties 
characteristic of a 400 kilometer altitude (see Table 3 ).

Figures 27 through 38 show the development of the flow field as the two plates approach one another. The top plate is held constant at -50 normalized potential and the bottom plate is floating. The figures are in groups of four showing $u, v, \rho$, and $\phi$ contours for plate separations of 24,9 , and 4 Debye lengths.

For large separations, the potential of the floating plate is unaffected by the presence of the top plate and takes on a potential characteristic of a balance between ambient ionic and electronic currents. As the two plates become closer, two main adjustments occur. First, the potential field of the top plate begins to dominate the inner region, drawing nearly all the plasma supplied to the region to its inner surface and away from the bottom plate. As a result, the current to the inner surface of the bottom plate decreases, and a very small separation distances goes to zero. Referring to equations (4.6) and (4.7) used for the floating potential calculation it is seen that a decrease in the ionic current must be compensated by a more negative floating potential. Secondly, the more negative potential field created by the top plate envelopes the bottom plate for small separation distances. The current to the outer surface of the bottom plate increases due to an increase in the perpendicular velocity caused by the larger potential gradient. Again referring to a current balance calculation, an increase in ionic current is compensated by a less negative floating 
Fig. 27

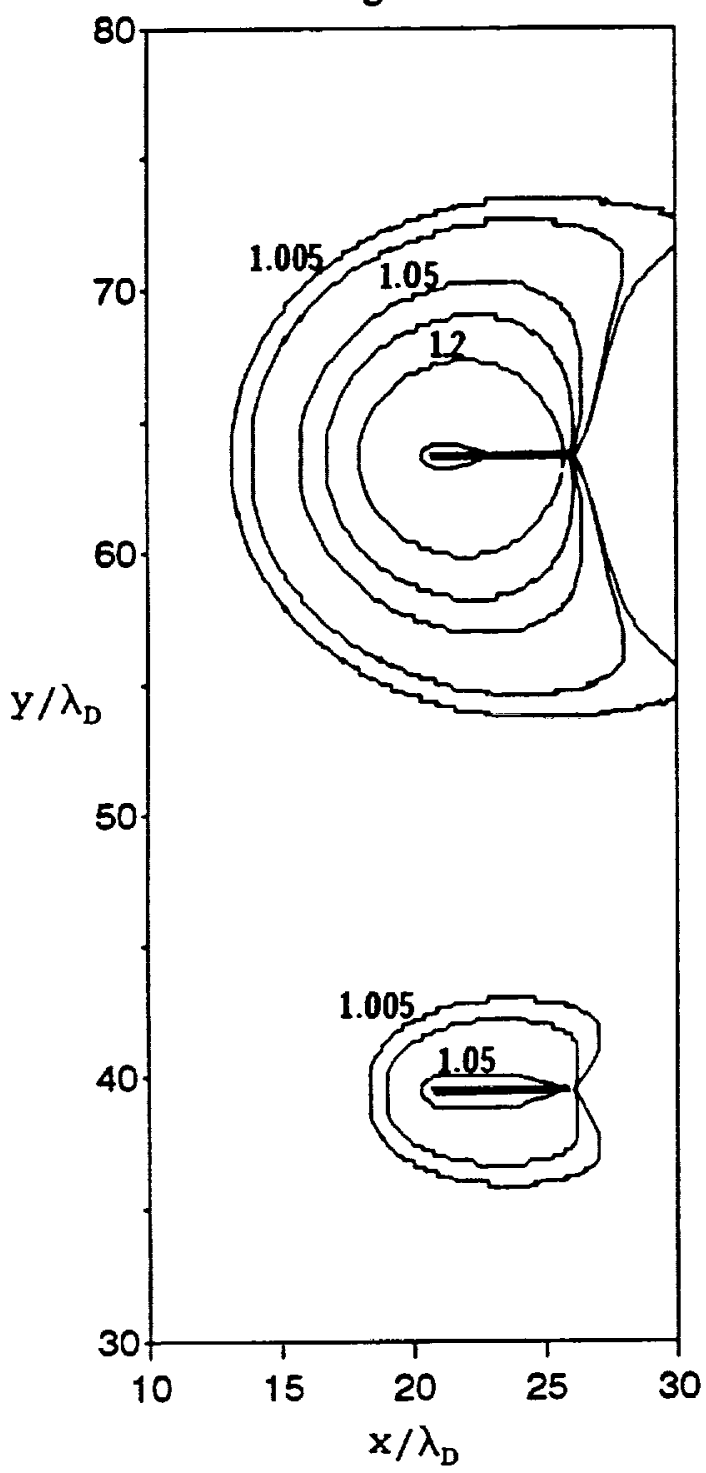

Fig. 28

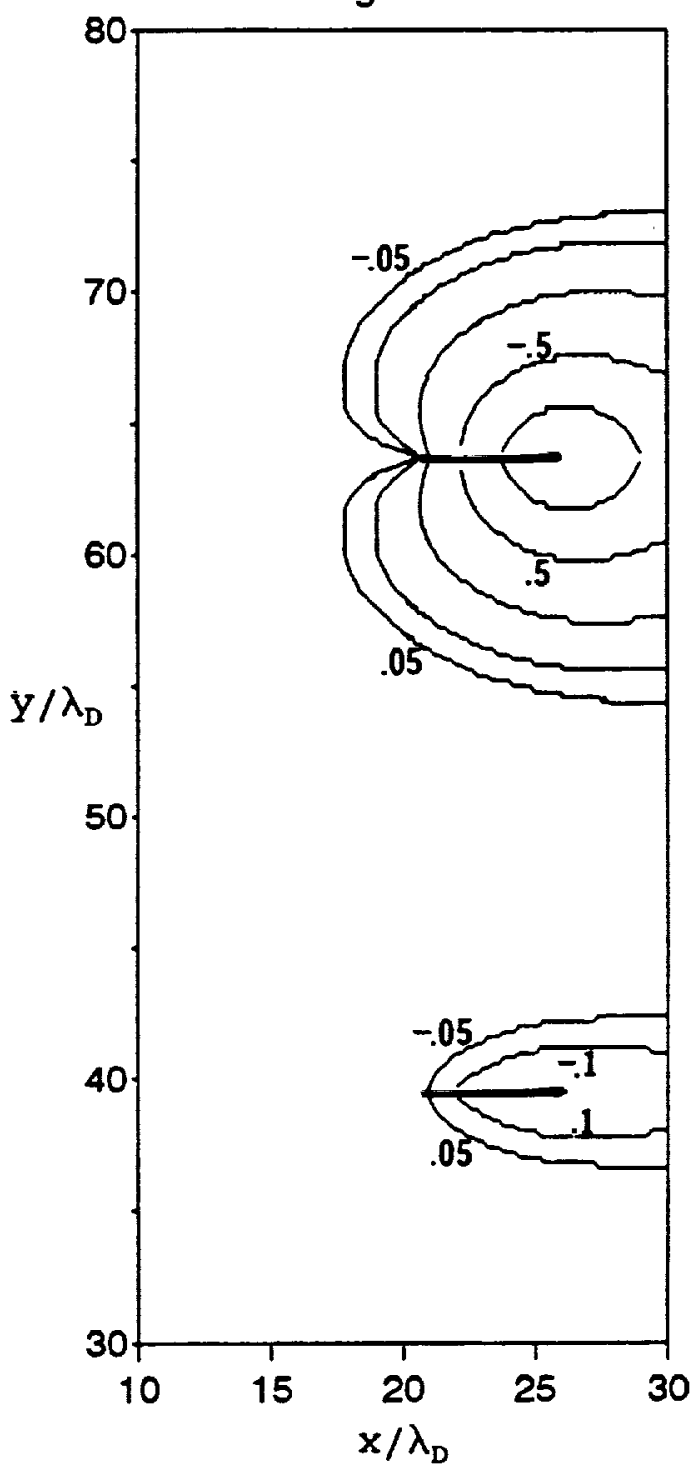

Figure 27. $u$ velocity contours for the case of an electrically floating bottom plate: $d_{x p p}=24 \lambda_{D}, d_{p}=5 \lambda_{D}, \phi_{p(t o p)}=-50$, $\phi_{\mathrm{f}(\mathrm{bat})}=-4.3, \mathrm{u}_{\infty}=7800 \mathrm{~m} / \mathrm{s}, \mathrm{T}_{\mathrm{e}}=0.2 \mathrm{eV}, \mathrm{m}_{\mathrm{i}}=16$ amu Contour values: $u / u_{\infty}=1.005,1.01,1.05,1.1,1.2$, 1.6 from the freestream toward the plates

Figure 28. $v$ velocity contours for the case of an electrically floating bottom plate: $\alpha_{\text {sep }}=24 \lambda_{D}, d_{p}=5 \lambda_{D}, \phi_{p(\text { top })}=-50$, $\phi_{f(b x)}=-4.3, u_{\infty}=7800 \mathrm{~m} / \mathrm{s}, T_{e}=0.2 \mathrm{eV}, m_{i}=16 \mathrm{amu}$

Contour values:v/ $u_{\infty}= \pm .05, \pm .1, \pm .25, \pm .5, \pm .75$ positive values below the plates, negative values above from the freestream toward the plates 
Fig. 29

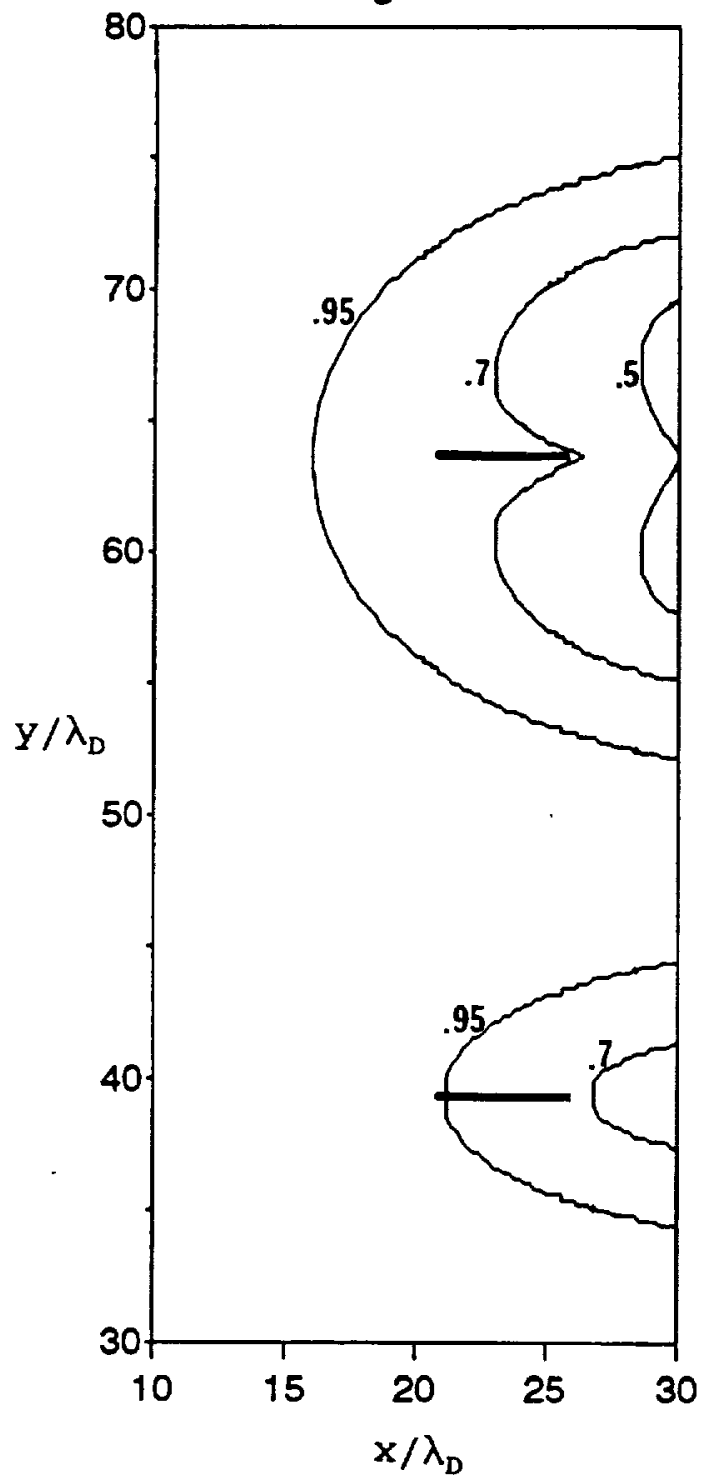

Fig. 30

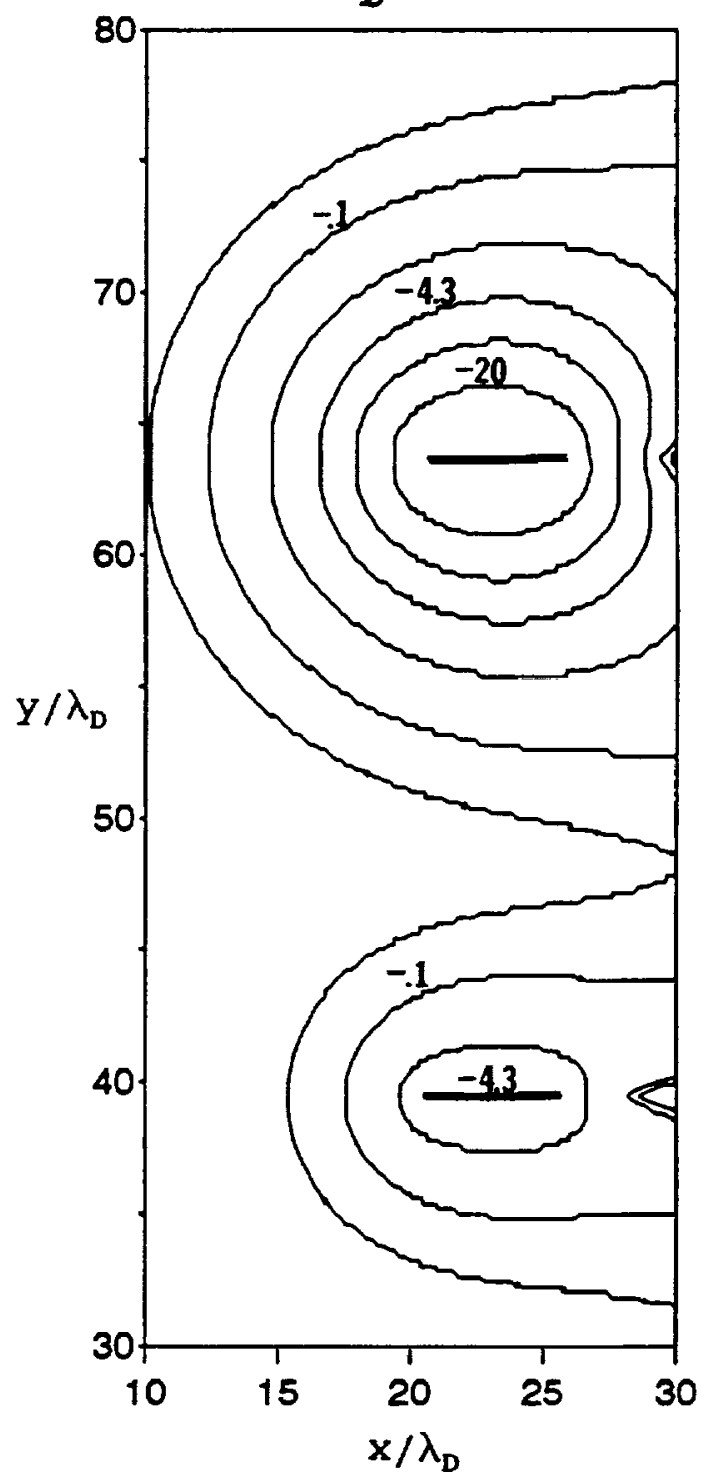

Figure 29. Ionic density contours for the case of an electrically floating bottom plate: $d_{s c p}=24 \lambda_{D}$, $d_{p}=5 \lambda_{D}, \phi_{p(l o p)}=-50, \phi_{f(b o t)}=-4.3, u_{\infty}=7800 \mathrm{~m} / \mathrm{s}$, $\mathrm{T}_{\mathrm{e}}=0.2 \mathrm{eV}, \mathrm{m}_{\mathrm{i}}=16$ amu

Contour values: $n_{i} / n_{\infty}=.95, .7, .5$ from the freestream toward the plates

Figure 30. Electric potential contours for the case of an electrically floating bottom plate: $d_{s \in p}=24 \lambda_{D}$, $d_{p}=5 \lambda_{D}, \phi_{p(L o p)}=-50, \phi_{f(b o t)}=-4.3, u_{\infty}=7800 \mathrm{~m} / \mathrm{s}$, $\mathrm{T}_{\mathrm{e}}=0.2 \mathrm{eV}, \mathrm{m}_{\mathrm{i}}=16$ amu

Contour values: $\phi=-.01,-.1,-1,-4.3,-10,-20$, from the freestream toward the plates 


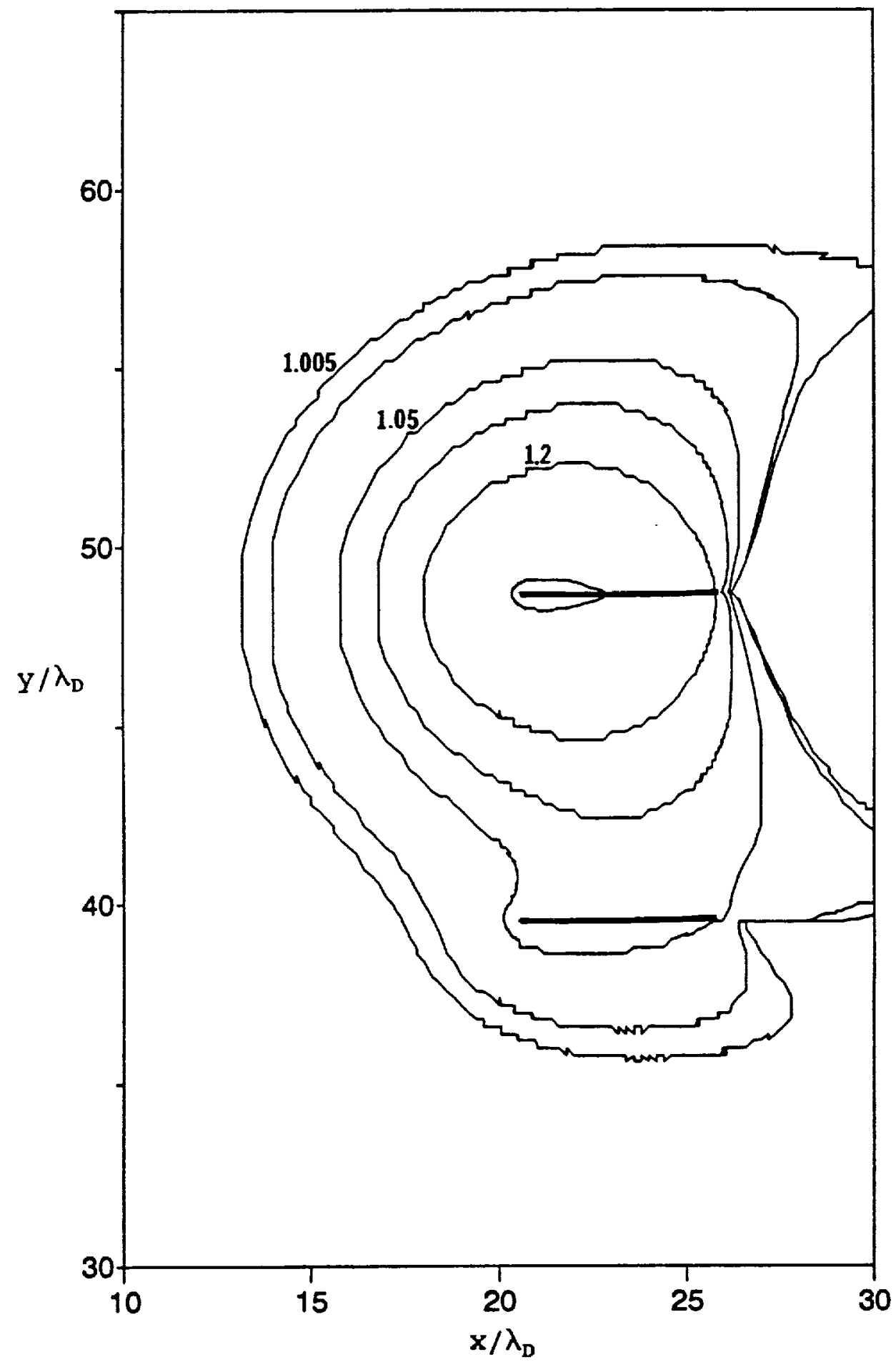

Figure 31. u velocity contours for the case of an electrically floating bottom plate: $d_{\operatorname{sep}}=9 \lambda_{D}, d_{p}=5 \lambda_{D}, \phi_{p(\text { cop })}=-50$, $\phi_{f(b \alpha)}=-4.6, u_{\infty}=7800 \mathrm{~m} / \mathrm{s}, T_{c}=0.2 \mathrm{eV}, m_{i}=16 \mathrm{amu}$

Contour values: $u / u_{\infty}=1.005,1.01,1.05,1.1,1.2$, 1.6 from the freestream toward the plates 


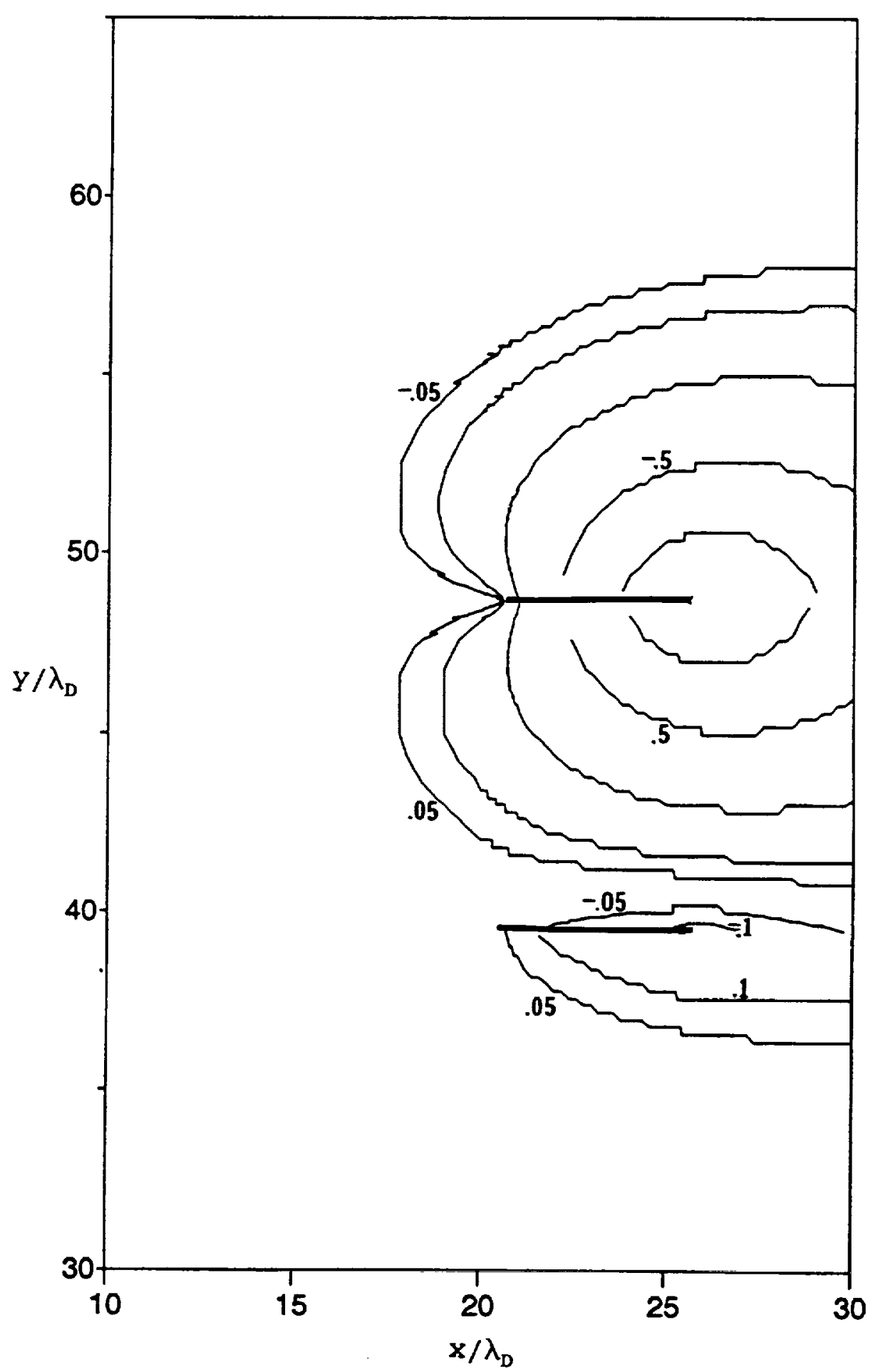

Figure 32. $v$ velocity contours for the case of an electrically floating bottom plate: $d_{\text {sep }}=9 \lambda_{D}, d_{p}=5 \lambda_{D}, \phi_{p(\text { lop })}=-50$, $\phi_{f(b a t)}=-4.6, u_{\infty}=7800 \mathrm{~m} / \mathrm{s}, \mathrm{T}_{\mathrm{e}}=0.2 \mathrm{eV}, \mathrm{m}_{\mathrm{j}}=16 \mathrm{amu}$

Contour values: $v / u_{\infty}= \pm .05, \pm .1, \pm .25, \pm .5, \pm .75$ positive values below the plates, negative values above from the freestream toward the plates 


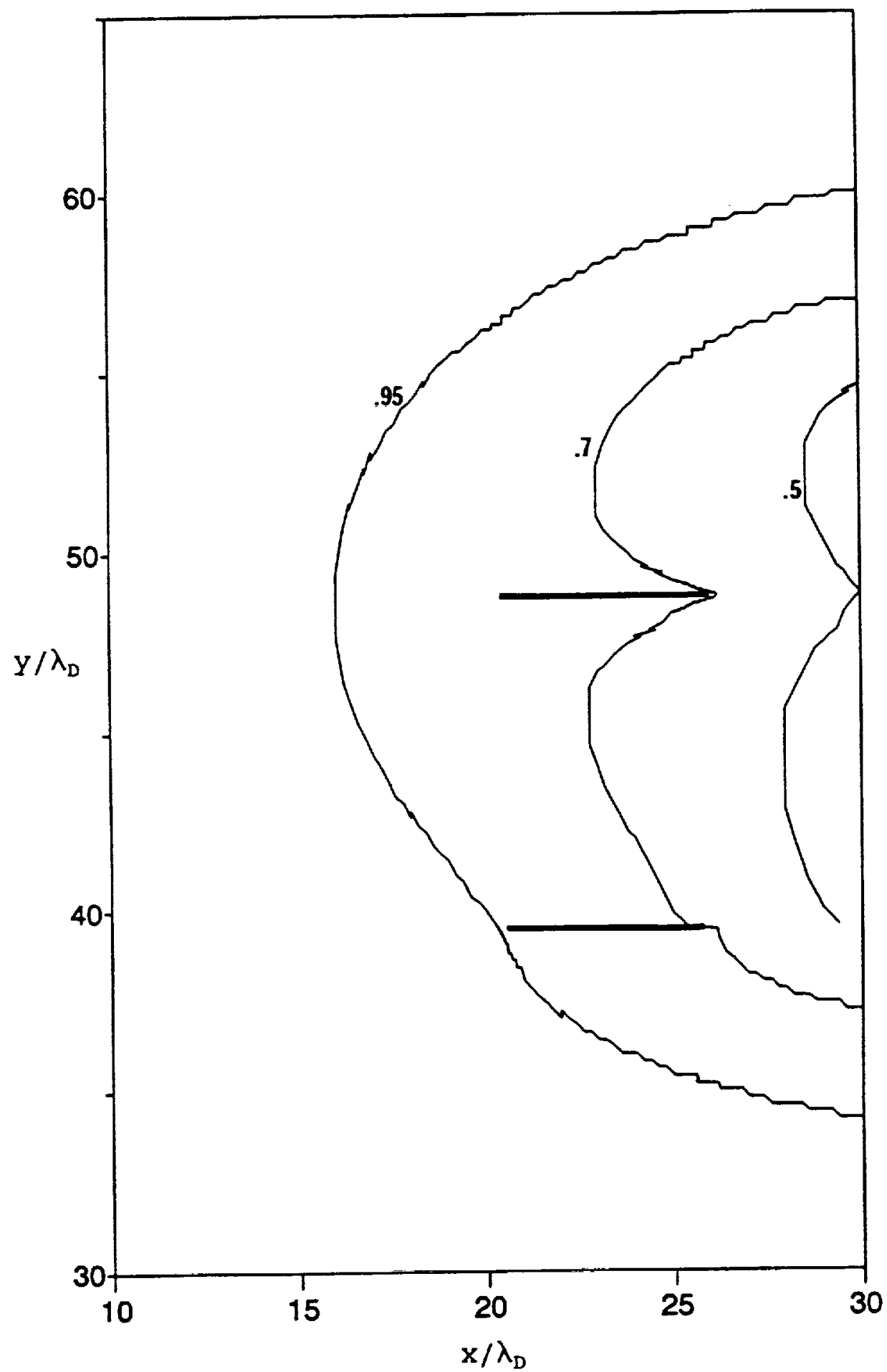

Figure 33. Ionic density contours for the case of an electrically floating bottom plate: $d_{s c p}=9 \lambda_{D}, d_{p}=5 \lambda_{D}$, $\phi_{\mathrm{p}(\mathrm{Lop})}=-50, \phi_{\mathrm{f}(\mathrm{box})}=-4.6, \mathrm{u}_{\infty}=7800 \mathrm{~m} / \mathrm{s}, \mathrm{T}_{\mathrm{c}}=0.2 \mathrm{eV}$, $\mathrm{m}_{\mathrm{i}}=16 \mathrm{amu}$

Contour values: $n_{i} / n_{\infty}=.95, .7, .5$ from the freestream toward the plates 


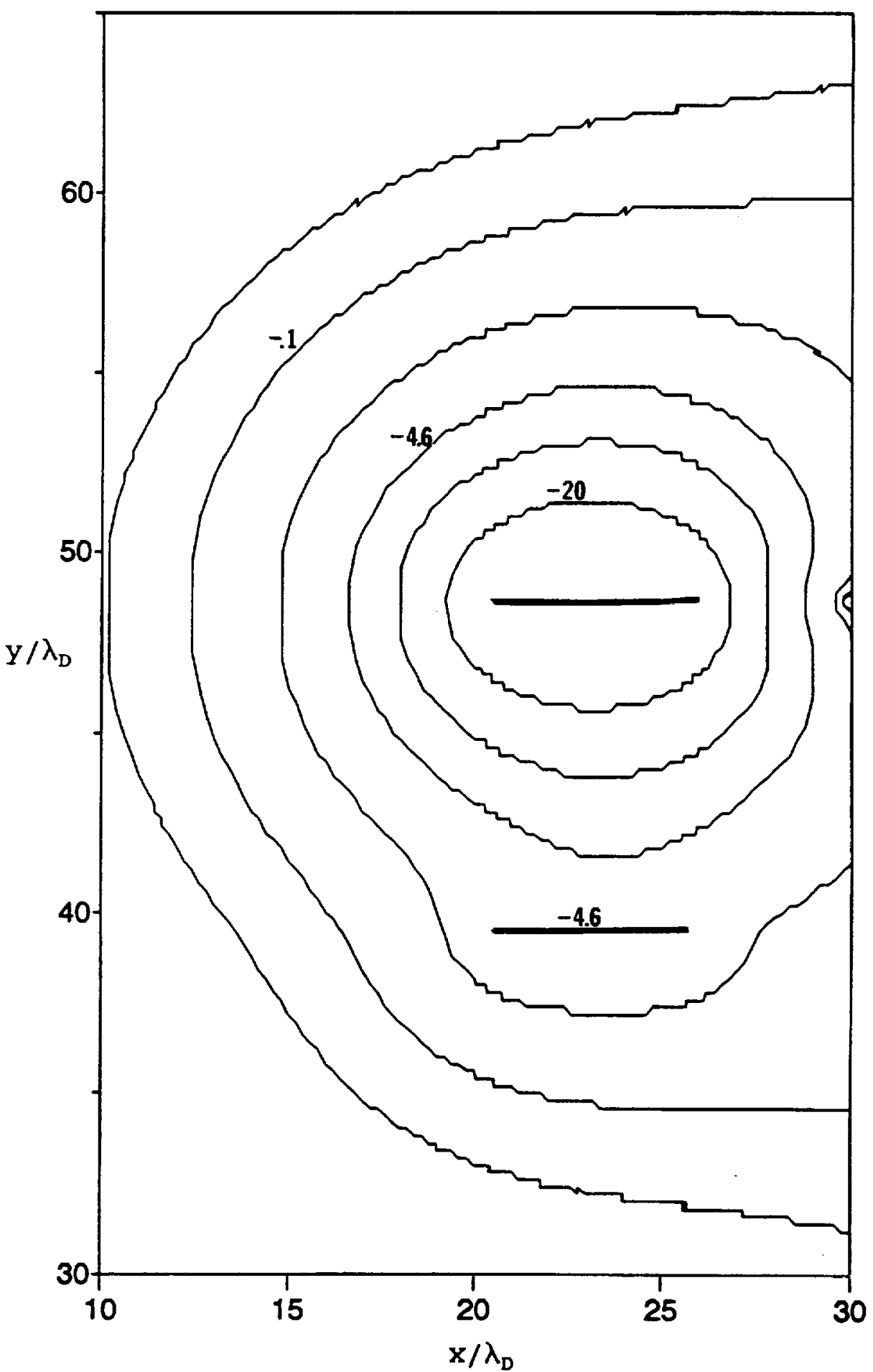

Figure 34. Electric potential contours for the case of an electrically floating bottom plate: $d_{2 c p}=9 \lambda_{D}$, $d_{p}=5 \lambda_{D}, \phi_{p(t o p)}=-50, \phi_{f(b o d)}=-4.6, u_{\infty}=7800 \mathrm{~m} / \mathrm{s}$, $\mathrm{T}_{\mathrm{c}}=0.2 \mathrm{eV}, \mathrm{m}_{\mathrm{j}}=16 \mathrm{amu}$

Contour values: $\phi=-.01,-.1,-1,-4.6,-10,-20$, from the freestream toward the plates 


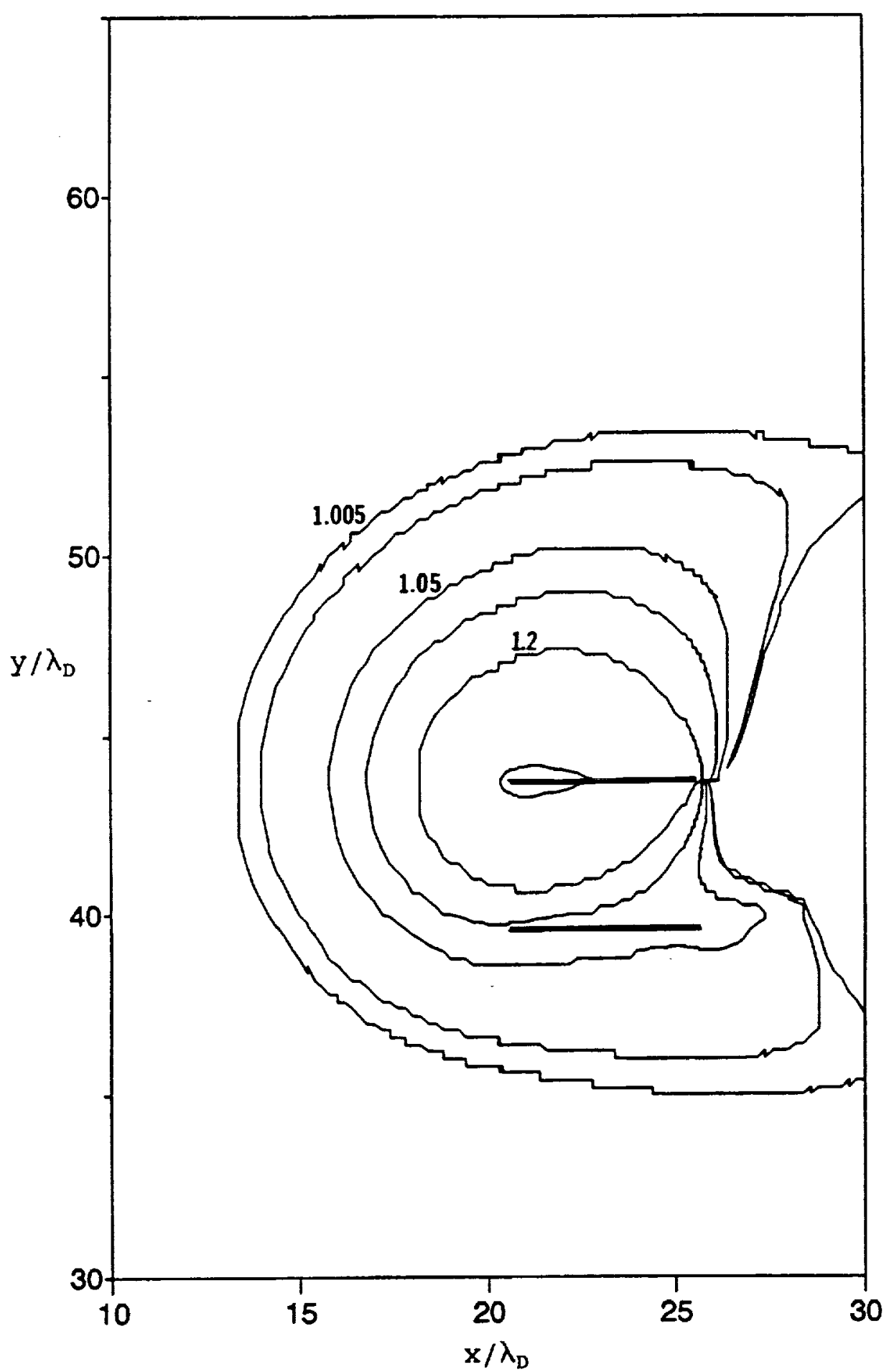

Figure 35. u velocity contours for the case of an electrically floating bottom plate: $d_{\operatorname{sep}}=4 \lambda_{D}, d_{p}=5 \lambda_{D}, \phi_{p(\operatorname{cop})}=-50$, $\phi_{\mathrm{f}(\mathrm{bor})}=-4.75, \mathrm{u}_{\infty}=7800 \mathrm{~m} / \mathrm{s}, \mathrm{T}_{\mathrm{c}}=0.2 \mathrm{eV}, \mathrm{m}_{\mathrm{i}}=16 \mathrm{amu}$ Contour values: $u / u_{\infty}=1.005,1.01,1.05,1.1,1.2$, 1.6 from the freestream toward the plates 


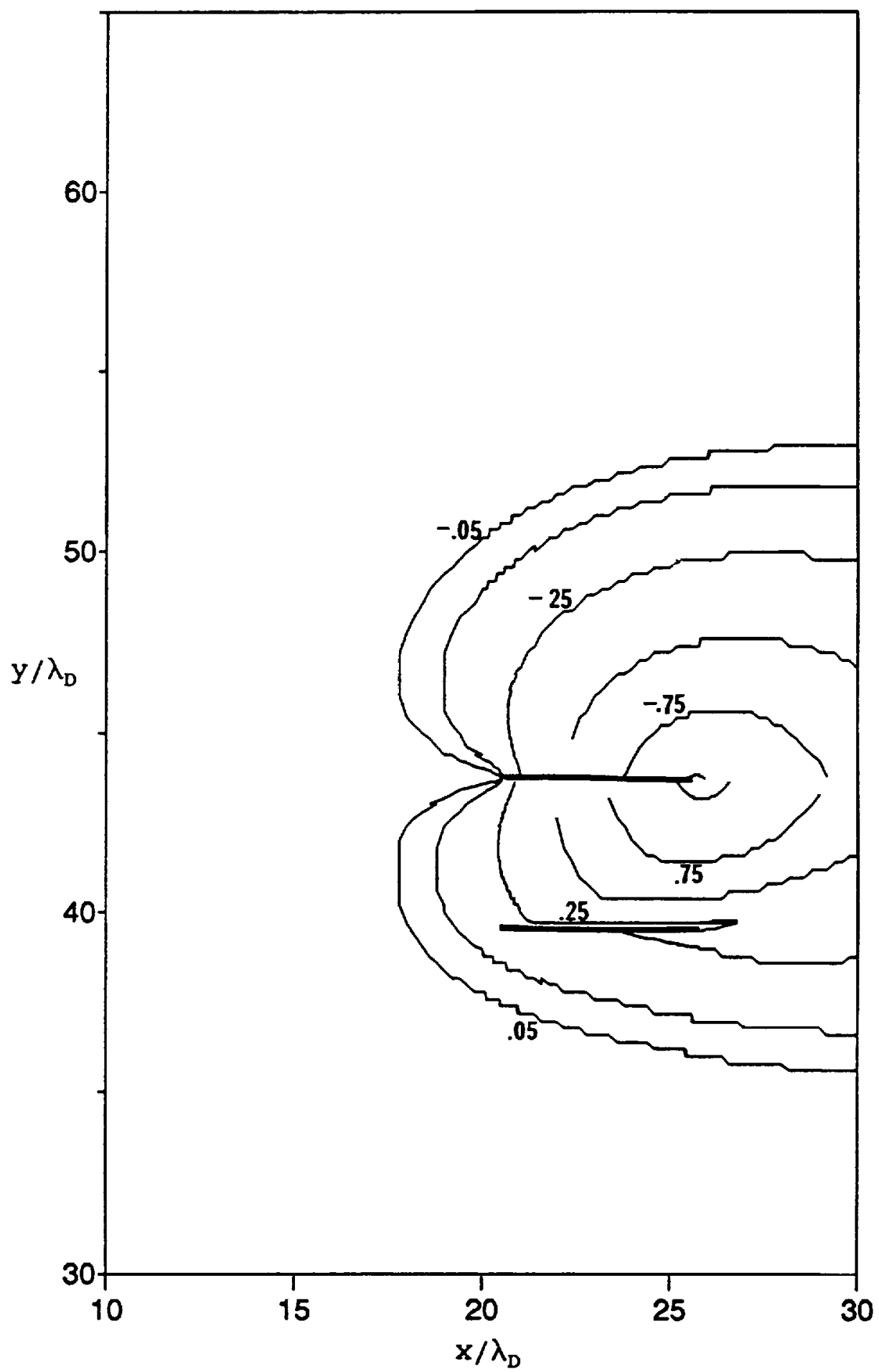

Figure 36. $v$ velocity contours for the case of an electrically floating bottom plate: $d_{\text {sep }}=4 \lambda_{D}, d_{p}=5 \lambda_{D}, \phi_{p(L o p)}=-50$, $\phi_{f(b a x)}=-4.75, u_{\infty}=7800 \mathrm{~m} / \mathrm{s}, \mathrm{T}_{\mathrm{e}}=0.2 \mathrm{eV}, \mathrm{m}_{\mathrm{i}}=16 \mathrm{amu}$

Contour values: $v / u_{\infty}= \pm .05, \pm .1, \pm .25, \pm .5, \pm .75$, \pm 1 positive values below the plates, negative values above from the freestream toward the plates 


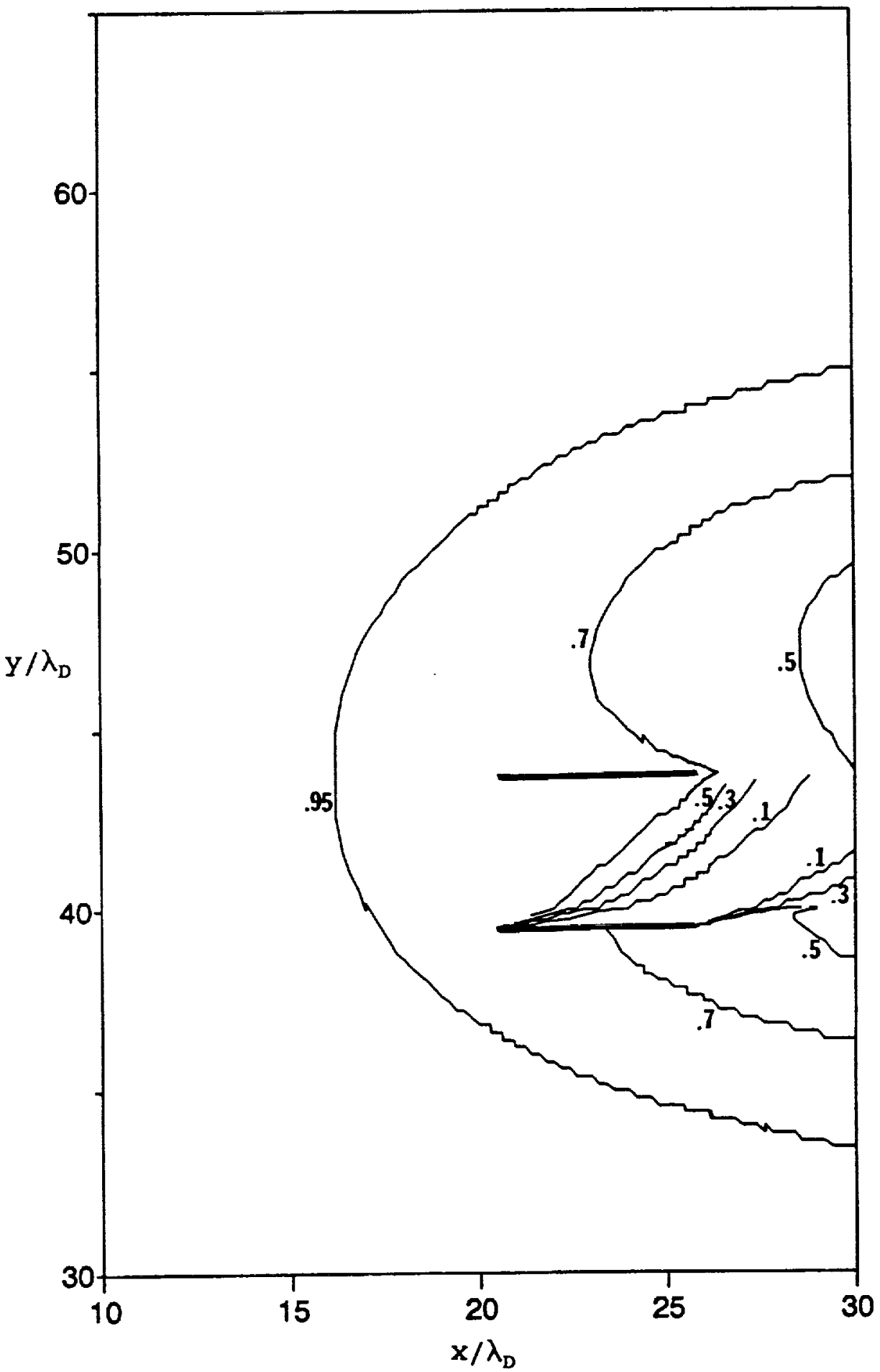

Figure 37. Ionic density contours for the case of an electrically floating bottom plate: $d_{x c p}=4 \lambda_{D}$. $\mathrm{d}_{\mathrm{p}}=5 \lambda_{\mathrm{D}}, \phi_{\mathrm{p}(\mathrm{top})}=-50, \phi_{\mathrm{rbot})}=-4.75, \mathrm{u}_{\infty}=7800 \mathrm{~m} / \mathrm{s}$, $\mathrm{T}_{\mathrm{e}}=0.2 \mathrm{eV}, \mathrm{m}_{\mathrm{i}}=16 \mathrm{amu}$

Contour values: $n_{i} / n_{\infty}=.95, .7, .5, .3, .1$ 


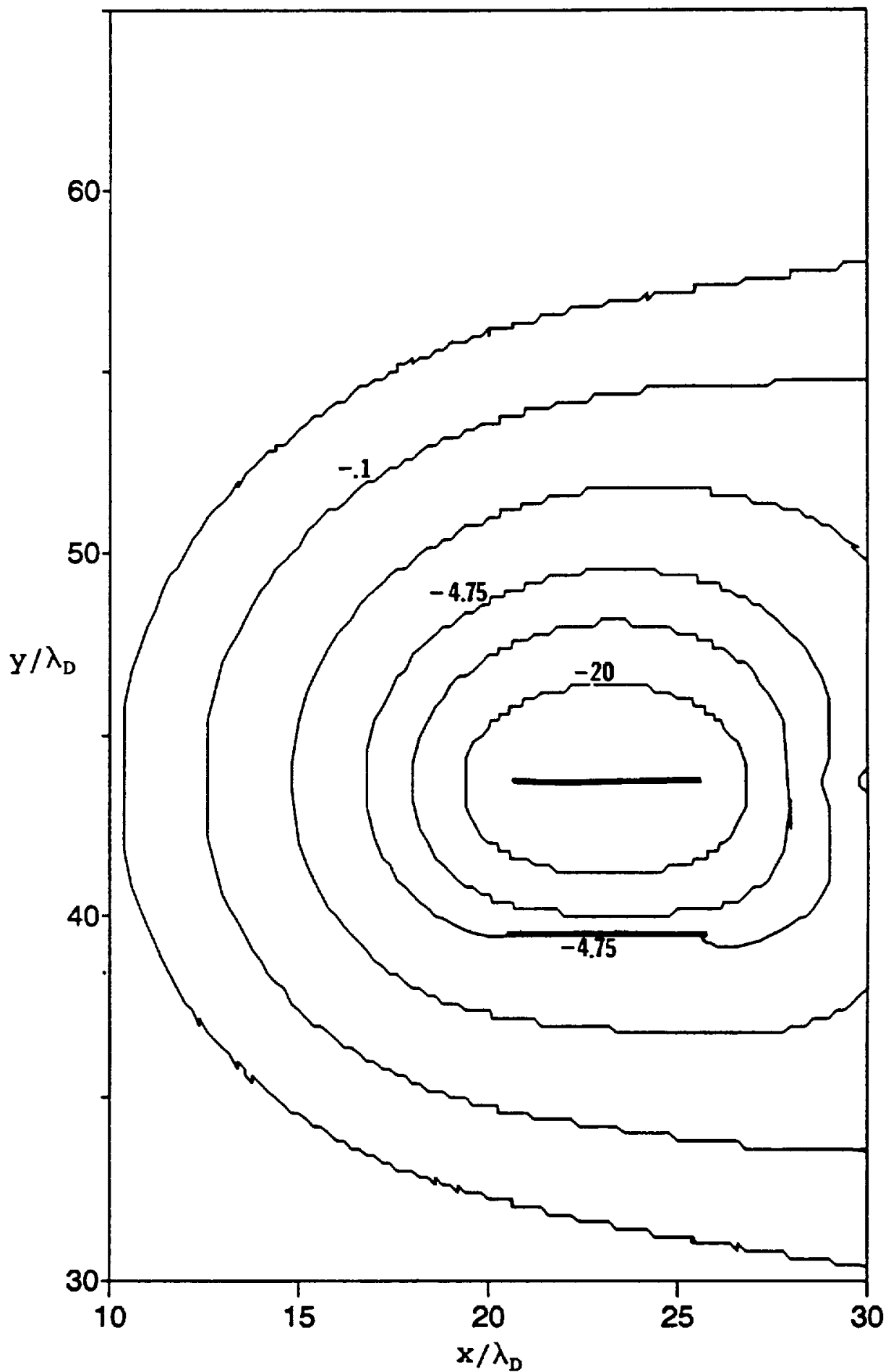

Figure 38. Electric potential contours for the case of an electrically floating bottom plate: $\alpha_{s c p}=4 \lambda_{D}$, $d_{p}=5 \lambda_{D}, \phi_{p(\text { lop })}=-50, \phi_{\text {f(box })}=-4.75, u_{\infty}=7800 \mathrm{~m} / \mathrm{s}$, $T_{c}=0.2 \mathrm{eV}, \mathrm{m}_{\mathrm{i}}=16 \mathrm{amu}$

Contour values: $\phi=-.01,-.1,-1,-4.75,-10,-20$, from the freestream toward the plates 
potential.

Figure 39 shows the negative floating potential of the bottom plate in normalized units versus separation distance in Debye lengths. At large separations, the floating potential is approximately 4.3 times the electron temperature which is characteristic of a flow about a solitary plate for the plasma conditions chosen. Figures 27 through 30 show that the flow field about the bottom plate is essentially unaffected by the presence of the top plate for this separation.

At midrange separations, (i.e. 8 to 12 Debye lengths) the potential field created by the top plate has not yet enveloped the bottom plate, but still draws much of the plasma supplied to the middle region to its inner surface. An overall decrease in current to the bottom plate results causing its floating potential to increase by approximately 10\%. Figures 31 through 34 show the flow field about the pair of plates for. a 9 Debye length separation. comparing the v-velocity contours of figure 32 to figure 28 it is seen that there will be a decrease in current to the inner surface of the lower plate because of a slower perpendicular velocity. At the same time, there is not much of an increase in current to the outer surface. The floating potential thus increases in order to impede more of the electrons and balance the overall current. The calculated floating potential value for this case is plotted as a contour in figure 34 to show that the bottom plate has not been fully enveloped in the potential field created by the top plate. 


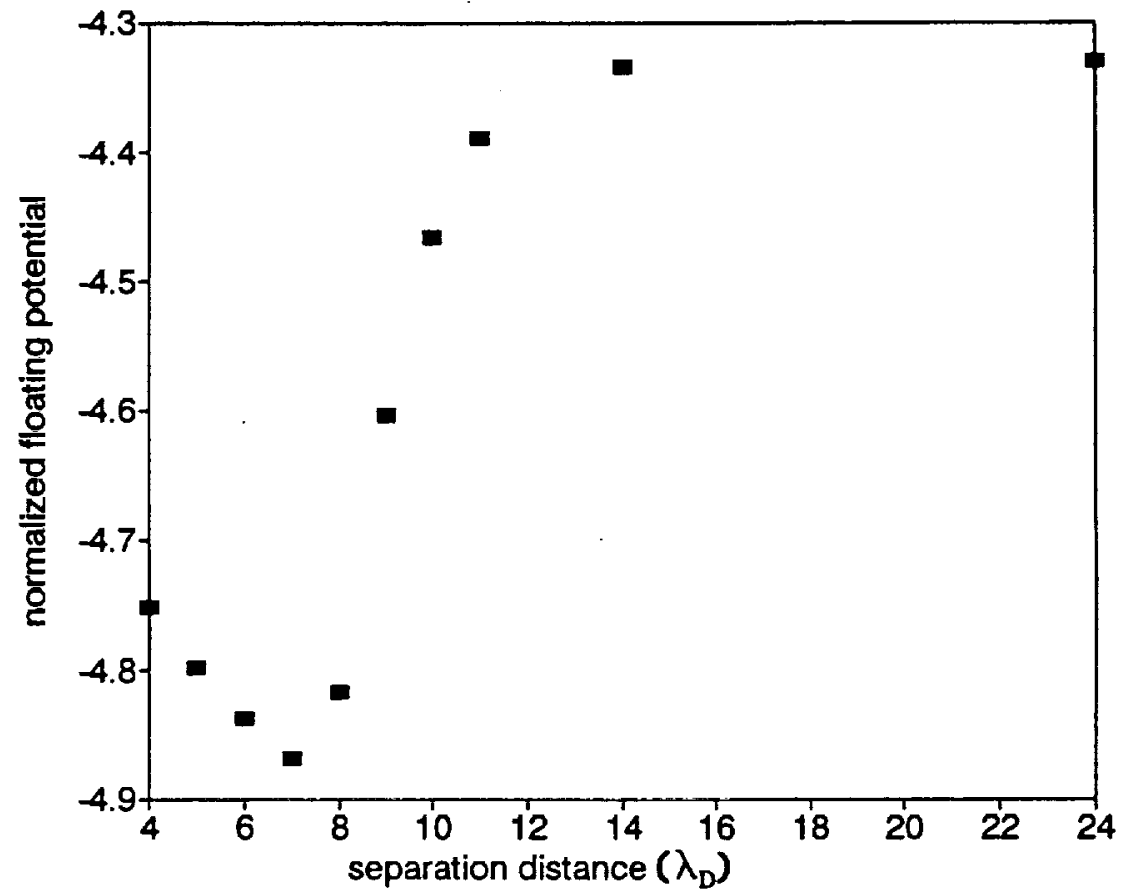

Figure 39. Floating potential of the bottom plate as a function of separation distance: $d_{p}=5 \lambda_{D}$, $\phi_{p(\text { Lop })}=-50, u_{\infty}=7800 \mathrm{~m} / \mathrm{s}, \mathrm{T}_{\mathrm{e}}=0.2 \mathrm{eV}, \mathrm{m}_{\mathrm{i}}=16 \mathrm{amu}$ 
At even closer distances, the current to the inner surface of the bottom plate is nearly zero. In figure 36 the v-velocity contour line adjacent to the inner surface of the lower plate has a positive value which means that the ions are being drawn away. Figure 37 shows the resulting density contours. The density rapidly decreases in the flow direction between the plates as the ions are being drawn away from the bottom plate and absorbed by the top plate. The potential field created by the top plate, however, has fully enveloped the bottom plate as shown in figure 38 . The greater potential gradient causes an increase in current to the outer surface of the lower plate by increasing the perpendicular velocity. Just downstream of the lower plate, this causes an increase in density in the flow direction as the near-void region is replenished by the accelerated ions as shown in figure 37 . The increase in current to the outer surface of the lower plate is enough to raise (i.e. less negative) the floating potential slightly.

The adjustment by the bottom plate is not a monotonic one as it would be if it charged increasingly negative as it approached the top plate. Instead, there is an oscillation in the floating potential as a lack and then an increase in ionic current causes the bottom plate to charge more and then less negative respectively. The increase (i.e. less negative) in the floating potential is expected to continue for still smaller separations until the two plates come in contact to form a single system at which time the bottom plate will 
rapidly charge to a potential near that of the top plate. The computer code developed could not be used for the smaller separations because of the near-zero density above the lower plate that would result.

When studying the docking of two spacecraft, the problem was modeled in a quasi-static manner. The flow field was calculated at different separation distances independently. This assumes that the electric sheath structure of the two plates establishes itself on a time scale much less than the movement of the plates toward one another.

An estimate of the time scale can be made by assuming that the sheath is established in the time required by an ion to pass through the sheath [ref. 28]. At a constant acceleration and assuming the ion starts from rest, the time of flight can be estimated by $t_{f}=\left(2 d_{f} m_{i} / e E\right)^{1 / 2}$. Using the results from the single flat plate case study presented previously, a time scale on the order of $10^{-4}$ seconds is calculated where the distance of flight $d_{f}$ is taken to be the sheath width and the electric field is the value at the sheath edge $(E=0.04 \mathrm{~V} / \mathrm{m})$. This is a conservative estimate considering that the electric field strength increases closer to the plates. Assuming that docking spacecraft will approach each other at no more than $0.01 \mathrm{~m} / \mathrm{s}$ just prior to contact, the time during which a spacecraft five Debye lengths long is at a given point in space is on the order of a second. This simple analysis shows that the quasi-static assumption is appropriate. 
In the analysis, the plates were assumed to be metallic. This allowed a single electric potential to be defined for the entire plate surface. If the plates were composed of a dielectric material, however, the potential need not be constant since charge cannot distribute itself freely.

The flow field about a dielectric plate would be different than those found here, especially in the case of spacecraft docking. It was found that the flow field was not symmetric about the axes of the plates for small separation distances. In the case of a metallic plate, the lack of symmetry caused the floating potential of the bottom plate to oscillate upon approach. For a dielectric plate, there would no longer be an oscillation. The inner surface would charge continuously to a high negative potential due to the lack of ionic current, and vice-versa for the outer surface. The potential difference would no longer be between the top and bottom plates but between the inner and outer surfaces of the bottom plate. This type of charging, known as differential charging, can result in electrostatic discharges between surfaces, and ultimately interfere with spacecraft operation [ref. 29]. 
CHAPTER V

CONCLUSION

The steady state interaction of two electrically biased parallel plates immersed in a flowing plasma characteristic of low earth orbit is studied numerically. Fluid equations are developed to describe the motion of the cold positively charged plasma ions, and are solved using finite-differences in two dimensions on a Cartesian grid. A variable and staggered grid is employed. The behavior of the plasma electrons is assumed to be described by the Maxwell-Boltzmann distribution.

Results are compared to an analytical and a particle simulation technique for a simplified flow geometry consisting of a single negatively biased plate. Comparison of the extent of the electrical disturbance into the flowing plasma and the magnitude of the electrical current collected by the plate is very good.

The contribution of the present study is on the interaction between two negatively biased parallel plates. The first case study presented describes the interaction of 
equally biased plates. The separation distance at which the electric current collected by either plate decreases by five and twenty percent is determined as a function of applied potential. The percent decreases were based on a noninteracting case. It is found that the relationship between separation distance and applied potential is not linear. Rather, there is a rapid change at less negative potentials but levels off at greater distances for larger negative potentials. The decrease in the overall current is caused by a decrease in ionic density in the region between the plates. As the separation between the plates decreases, the plates collect the ions at a faster rate than they are supplied to the middle region by the oncoming plasma flow.

These results can be applied by space shuttle experimenters who need to know if the plasma flow field around their experiment will be affected by the electric potential of the shuttle.

The second case study presented simulates the docking of spacecraft in orbit. Two plates of unequal potential are moved toward one another in a quasi-static manner. One plate is held at a large negative potential while the other floats electrically in the resulting potential field. This problem is applicable to the docking of the shuttle with the proposed space station. The space station will have a relatively large constant negative potential due to solar array operation, and the shuttle will attain a potential determined mainly by a balance of ambient current. The potential of the floating 
plate is determined as a function of separation between the plates.

As the two plates approach one another, there is a decrease in current to the inner surface of the floating plate. This causes its potential to increase negatively. This trend reverses itself, however, as the plates get even closer due to an increase in current to the outer surface of the floating plate. The floating plate does not charge continuously negative as it approaches the other more negatively biased plate. Instead, it charges more and then less negative as ionic current decreases and then increases respectively upon approach. When the plates come into contact, it is expected that the electrically floating plate will charge rapidly negative to a potential near that of the other plate.

The computer code developed could not simulate the actual contact of the two plates. This is because the program cannot account for regions of zero density which occur when the two plates come within a couple Debye lengths of each other.

The limitation of the present approach is its inability to deal with regions of zero density where the continuum assumption fails. A way to circumvent this problem would be to deal with momentum as the fundamental variable instead of velocity and mass separately. Regions of zero momentum could then be viewed either as having no velocity or no mass, and the influence of those regions could be propagated correctly. When the variables are dealt with separately, situations can 
occur where there is no mass but a velocity field still exists especially in the present study where mass is being absorbed by the plates.

overall, the results from the study look promising. The ability of the continuum approach to model an essentially collisionless flow problem is good. In future work, dealing with momentum as the fundamental variable is the first step. Also, the concepts of temperature and pressure need to be incorporated so that there are other mechanisms for the dissipation of ionic density concentrations. The results presented should also apply to three-dimensional problems, but only for the limited range of geometrical configurations dealt with here. 


\section{REFERENCES}

1. Tanenbaum, B. Samuel, Plasma Physics, McGraw-Hill, New York, 1967.

2. "Spacecraft/Plasma Interactions and Electromagnetic Effects in LEO and Polar Orbit," Culham Laboratory, UK, March 1989.

3. de Leeuw, J.H., 'A Brief Introduction to Ionospheric Aerodynamics,' Proceedings of the Fifth International Symposium on Rarefied Gas Dynamics, edited by C.L. Brundin, Vol. II, Academic Press, New York, 1967, pp. 1561-1586.

4. Stone, Nobie H., 'The Aerodynamics of Bodies in a Rarefied Ionized Gas with Applications to spacecraft Environmental Dynamics,' NASA TP-1933, 1981.

5. Chen, F.F., "Electric Probes," Plasma Diagnostics Techniques, Academic Press, New York, 1965.

6. Chung, P.M., Talbot, L., Touryan, K.J., 'Part 2 . Continuum Probes,' AIAA Journal, Vol. 12, No. 2, Feb. 1974, pp. 144-154.

7. Holt, E.H., Haskell, R.E., Foundations of Plasma Dynamics, Macmillan Company, New York, 1965.

8. Su, C.H., "Compressible Plasma Flow over a Biased Body," AIAA Journal, Vol. 3, No. 5, May 1965, pp. 842-848.

9. Smy, P.R., 'The Use of Langmuir Probes in the Study of High Pressure Plasmas,' Advances in Physics, Vol. 25, No. 5, pp. 517-553.

10. Su, C.H., Lam, S.H., "Continuum Theory of Spherical Electrostatic Probes," Physics of Fluids, Vol. 6, No. 10, oct. 1963, pp. 1479-1491.

11. Cohen, I.M., "Asymptotic Theory of Spherical Electrostatic probes in a slightly Ionized, collisionDominated Gas," Physics of Fluids, Vol. 6, No. 10, Oct. 1963, pp. 1492-1499.

12. Lam, S.H., "A General Theory for the Flow of Weakly Ionized Gases," AIAA Journal, Vol. 2, No. 2, Feb. 1964, pp. 256-262. 
13. Stahl, N., Su, C.H., "Theory of Continuum Flush Probes," Physics of Fluids, Vol. 14, No. 7, July 1971, pp. 13661376 .

14. Giles, C.R., clements, R.M., Smy, P.R., 'Flush Probe Studies of Plasma Flow over a Flat Plate: Theory,' J. Phys. D: Appl. Phys., Vol. 12, 1979, pp. 1685-1697.

15. Johnson, R.A., De Boer, P.C.T., 'Theory of Ion Boundary Layers,' AIAA Journal, Vol. 10, May 1972, pp. 664-670.

16. Russo, A.J., Touryan, K.J., "Experimental and Numerical Studies of Flush Electrostatic Probes in Hypersonic Ionized Flows: II. Theory," AIAA Journal, Vol. 10, No. 12, Dec. 1972, pp. 1675-1678.

17. Chung, Paul M., Blankenship, Victor D., 'Theory of Electrostatic Double Probe Comprised of Two Parallel Plates,' AIAA Journal, Vol. 4, No. , March 1966, pp. 442450 .

18. Vitello, P., cerjan, C., Braun, D., 'Flow: A TwoDimensional Time-Dependent Hydrodynamical Ion Extraction Model,' Phys. Fluids B, Vol. 4, No. 6, June 1992, pp. 1447-1456.

19. Kraus, Lester, Watson, Kenneth M., 'Plasma Motion Induced by Satellites in the Ionosphere,' Physics of Fluids, Vol. 1 , No. 6, Nov.-Dec. 1958, pp. 480-488.

20. Lam, S.H., 'On the Capabilities of the cold-Ion Approximation in collisionless Plasma Flows,' Proceedings of the Fifth International symposium on Rarefied Gas Dynamics, edited by C.L. Brundin, Vol. II, Academic Press, New York, 1967, pp. 1603-1621.

21. Lam, S.H., Greenblatt, M., 'Flow of a Collisionless Plasma over a Cone," AIAA Journal, Vol. 3, No. 10, oct. 1965, pp. 1850-1855.

22. Anderson, John D. Jr., Fundamentals of Aerodynamics, 2nd ed., McGraw-Hill, 1991.

23. Hohl, Frank, Wood, George P., 'The Electrostatic and Electromagnetic Drag Forces on a Spherical satellite in a Rarefied Partially Ionized Atmosphere,' Proceeding of the 3rd International Symposium on Rarefied Gas Dynamics, edited by J.A. Laurmann, Vol. II, Academic Press, New York, 1963, pp. 45-64. 
24. Davis, A.H., Harris, I., 'Interaction of a Charged Satellite with the Ionosphere,' Proceedings of the 2nd International symposium on Rarefied Gas Dynamics, edited by L. Talbot, Academic Press, New York, 1961, pp. 691699.

25. Wang, J., Hastings, D.E., 'Ionospheric Plasma Flow over Large High-Voltage space Platforms. I: Ion-Plasma-Time Scale Interactions of a Plate at Zero Angle of Attack,' Phys. Fluids B, Vol. 4, No. 6, June 1992, pp. 1597-1614.

26. Anderson, Dale A., Tannehill, John C., Pletcher, Richard H. Computational Fluid Mechanics and Heat Transfer, Hemisphere Publishing Corporation, New York, 1984.

27. Chiu, Ing-Tsau, 'Prediction of Laminar Flows over a Rearward-facing step Using the Partially-Parabolized Navier-stokes Equation,' Master's Thesis, Mechnical Engineering Department, Iowa State University, Ames Iowa, 1984 .

28. Ginzburg, M.A., 'Double Electrical Layer on the Surface of a Satellite,' ARS Journal Supplement, Nov. 1962, pp. 1794-1800.

29. Purvis, Carolyn K., et.al., 'Design Guidelines for Assessing and Controlling Spacecraft Charging Effects,' NASA TP-2361, September 1984. 
Public reporting burden for this collection of information is estimated to average 1 hour per response, including the time for reviewing instructions, searching existing data sources, gathering and maintaining the data needed, and completing and revlewing the collection of information. Send comments regarding lhis burden estimate or any other aspect of this

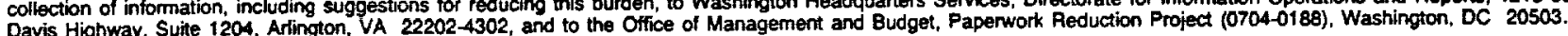

\begin{tabular}{|l|c|c|}
\hline 1. AGENCY USE ONLY (Leave blank) & $\begin{array}{c}\text { 2. REPORT DATE } \\
\text { April } 1993\end{array}$ & $\begin{array}{r}\text { 3. REPORT TYPE AND DATES COVERED } \\
\text { Final Contractor Report }\end{array}$
\end{tabular}

\section{TITLE AND SUBTITLE}

Ionospheric Plasma Flow About a System of Electrically Biased Flat Plates

WU-506-41-52

C-NAS3-25266

6. AUTHOR(S)

Joel L. Herr

7. PERFORMING ORGANIZATION MAME(S) AND ADDRESS(ES)

Sverdrup Technology, Inc.

8. PERFORMING ORgANIzATION REPORT NUMBER

Lewis Research Center Group

2001 Aerospace Parkway

E-7732

Brook Park, Ohio 44142

9. SPONSORING/MONITORING AGENCY NAMES(S) AND ADDRESS(ES)

10. SPONSORINGMONITORING AGENCY REPORT NUMBER

National Aeronautics and Space Administration

Lewis Research Center

NASA CR-191119

Cleveland, Ohio 44135-3191

11. SUPPLEMENTARY NOTES

Project Manager, Dale C. Ferguson, Power Technology Division, NASA Lewis Research Center, (216) 433-2298. This paper was submitted in partial fulfillment of the requirements for the degree Master's of Science in Mechanical Engineering to Cleveland State University, Cleveland, Ohio.

12a. DISTRIBUTION/AVAILABILITY STATEMENT

12b. DISTRIBUTION CODE

Unclassified - Unlimited

Subject Category 18

13. ABSTRACT Maximum 200 words,

The steady state interaction of two electrically biased parallel plates immersed in a flowing plasma characteristic of low earth orbit is studied numerically. Fluid equations are developed to describe the motion of the cold positively charged plasma ions, and are solved using finite-differences in two dimensions on a Cartesian grid. The behavior of the plasma electrons is assumed to be described by the Maxwell-Boltzmann distribution. Results are compared to an analytical and a particle simulation technique for a simplified flow geometry consisting of a single semi-infinite negatively biased plate. Comparison of the extent of the electrical disturbance into the flowing plasma and the magnitude of the current collected by the plate is very good. The interaction of two equally biased parallel plates is studied as a function of applied potential. The separation distance at which the current collected by either plate decreases by five and twenty percent is determined as a function of applied potential. The percent decreases were based on a non-interacting case. The decrease in overall current is caused by a decrease in ionic density in the region between the plates. As the separation between the plates decreases, the plates collect the ions at a faster rate than they are supplied to the middle region by the oncoming plasma flow. The docking of spacecraft in orbit is simulated by moving two plates of unequal potential toward one another in a quasi-static manner. One plate is held at a large negative potential while the other floats electrically in the resulting potential field. It is found that the floating plate does not charge continuously negative as it approaches the other more negatively biased plate. Instead, it charges more and then less negative as ionic current decreases and then increases respectively upon approach. When the two plates come into contact, it is expected that the electrically floating plate will charge rapidly negative to a potential near that of the other plate.

14. SUBJECT TERMS

Ionospheric aerodynamics; Dual spacecraft interactions; Plasma flow interactions

15. NUMBER OF PAGES 82

16. PRICE CODE A05

17. SECURITY CLASSIFICATION OF REPORT

Unclassified
18. SECURITY CLASSIFICATION OF THIS PAGE

Unclassified
19. SECURITY CLASSIFICATION OF ABSTRACT

Unclassified 